\title{
Pot-economical total synthesis of clinprost
}

\author{
Nariyoshi Umekubo, Yujiro Hayashi* \\ Department of Chemistry, Graduate School of Science, Tohoku University 6-3 Aramaki-Aza Aoba-ku, \\ Sendai, Miyagi 980-8579, Japan
}

SUPPORTING INFORMATION

Experimental procedures and Characterization data

\section{Table of Contents}

1. Materials and Methods Page S2

2. Experimental Procedures Page S3 - S24

2. 1. Asymmetric domino Michael/Michael reaction between ketone $\mathbf{5}$ and aldehyde $\mathbf{6 a}$. Page S3

2. 2. Dimethyl acetal protection of compound 9 Page S4

2. 3. Asymmetric domino Michael/Michael reaction between ketone $\mathbf{8}$ and aldehyde $\mathbf{6 b}$ Page S5

2. 4. Dimethyl acetal protection of compound $\mathbf{1 0}$ Page S6

2. 5. Deprotection of compound 12 Page $\mathrm{S} 7$

2. 6. One-pot procedure to synthesize compound $\mathbf{1 3}$ Page S8

2. 7. One-pot synthesis of compound $\mathbf{3 b}$ from compound $\mathbf{1 3}$ Page S9-S10

2. 8. One-pot synthesis of compound $\mathbf{1 9}$ from compound $\mathbf{3 b}$ Page S10-S11

2. 9. Suzuki-Miyaura reaction of compound 19 Page S11- S12

2. 10. Tamao-Fleming oxidation of compound 21. Page S12- S13

2. 11. Deprotection \& HWE reaction of compound 24 Page S13- S14

2. 12. Stereoselective reduction of compound $\mathbf{2 6}$ Page S14- S15

2. 13. Failed synthetic route of clinprost (Part 1) Page S16

2. 14. Asymmetric domino Michael/Michael reaction between ketone 8 and aldehyde $\mathbf{6 a}$. Page S17

2. 15. One-pot procedure to synthesize compound SI-5. Page S18

2. 16. One-pot synthesis of compound SI-6 from compound SI-5 Page S19

2. 17. Suzuki-Miyaura reaction of compound SI-6 Page S20- S21

2. 18. Deprotection \& HWE reaction of compound SI-7. Page S21- S22

2. 19. Failed synthetic route of clinprost (Part 2) .Page S22

2. 20. Deprotection \& HWE reaction of compound 21 Page S22- S23

2. 21. Synthesis of aldehyde 6 .Page S23-S24

2. 22. Information of compound $\mathbf{1 1}$ Page S24

3. References Page S24

Spectra for Compounds Page S25-S82 


\section{Materials and Methods}

\section{General Methods.}

General Remarks: All reactions were carried out under argon atmosphere and monitored by thin-layer chromatography using Merck 60 F254 precoated silica gel plates (0.25 mm thickness). Specific optical rotations were measured using a JASCO P-1020 polarimeter and a JASCO DIP-370 polarimeter. FT-IR spectra were recorded on a JASCO FT/IR-410 spectrometer and a Perkin Elmer spectrum BX FT-IP spectrometer. ${ }^{1} \mathrm{H}$ and ${ }^{13} \mathrm{C}$ NMR spectra were recorded on an Agilent-400 MR (400 MHz for ${ }^{1} \mathrm{H}$ NMR, $100 \mathrm{M}$ $\mathrm{Hz}$ for ${ }^{13} \mathrm{C} \mathrm{NMR}$ ) instrument. Data for ${ }^{1} \mathrm{H}$ NMR are reported as chemical shift ( $\left.\delta \mathrm{ppm}\right)$, integration multiplicity ( $\mathrm{s}=$ singlet, $\mathrm{d}=$ doublet, $\mathrm{t}=$ triplet, $\mathrm{q}=$ quartet, quintet $=$ quin, septet $=$ sep, $\mathrm{dd}=$ doublet of doublets, $\mathrm{ddd}=$ doublet of doublet of doublets, $\mathrm{dt}=$ double of triplets, $\mathrm{td}=$ triplet of doublets, $\mathrm{m}=$ multiplet, brs $=$ broad singlet), coupling constant (Hz), Data for ${ }^{13} \mathrm{C}$ NMR are reported as chemical shift. High resolution ESI-TOF mass spectra were measured by Themo Orbi-trap instrument. HPLC analysis was performed on a HITACHI Elite LaChrom Series HPLC, UV detection monitored at appropriate wavelength respectively, using CHIRALPACK $^{\circledR}$ IC $(0.46 \mathrm{~cm} \times 25 \mathrm{~cm})$ and CHIRALPACK ${ }^{\circledR} \mathrm{ID}(0.46 \mathrm{~cm} \times 25 \mathrm{~cm})$. 


\section{Experimental Procedures}

\subsection{Asymmetric domino Michael/Michael reaction between ketone 5 and aldehyde $6 a$}

$$
5 \mathrm{~mol} \%
$$

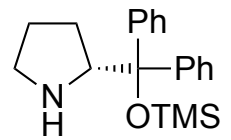

SI-1
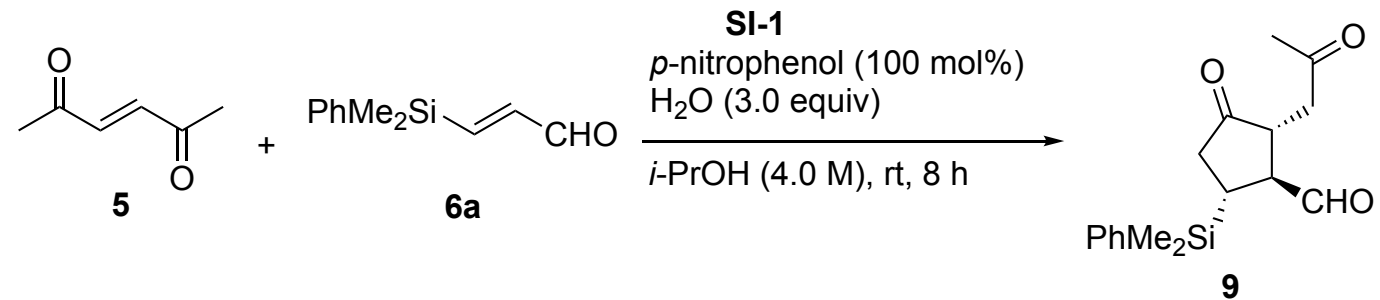

To a solution of ketone $\mathbf{5}^{[1]}(224 \mathrm{mg}, 2.0 \mathrm{mmol})$, aldehyde $\mathbf{6 a}^{[1]}(190 \mathrm{mg}, 1.0 \mathrm{mmol}), \mathrm{H}_{2} \mathrm{O}(540 \mu \mathrm{L}, 3.0$ $\mathrm{mmol})$ and $p$-nitrophenol (139 mg, $1.0 \mathrm{mmol})$ in $i$-PrOH $(250 \mu \mathrm{L})$, organocatalyst SI-1 (16.5 mg, $0.050 \mathrm{mmol})$ was added at room temperature. After stirring the reaction mixture at this temperature for $8 \mathrm{~h}$, the reaction mixture was directly purified by column chromatography on silica gel $(n$-Hexane: EtOAc $=6: 1)$ to give the target compound 9 (262 $\mathrm{mg}, 0.87 \mathrm{mmol}$ ) in $87 \%$ yield (single isomer).

(1S,2R,5R)-5-(Dimethyl(phenyl)silyl)-3-oxo-2-(2-oxopropyl)cyclopentane-1-carbaldehyde (9)<smiles>CC(=O)C[C@H]1C(=O)C[C@H](C)[C@@H]1C=O</smiles>

$\mathrm{PhMe}_{2} \mathrm{Si}$

Yield: $87 \%(262 \mathrm{mg})$

Physical State: Yellow oil

${ }^{1}$ H NMR $\left(\mathbf{C D C l}_{3}, 400\right.$ MHz) $\delta 9.38(\mathrm{~d}, 1 \mathrm{H}, J=2.4 \mathrm{~Hz}), 7.48-7.50(\mathrm{~m}, 2 \mathrm{H}), 7.34-7.40(\mathrm{~m}, 3 \mathrm{H}), 2.79(\mathrm{dd}, 1 \mathrm{H}$, $J=3.6,18.8 \mathrm{~Hz}), 2.61-2.75(\mathrm{~m}, 3 \mathrm{H}), 2.47(\mathrm{ddd}, 1 \mathrm{H}, J=0.80,8.8,18.8 \mathrm{~Hz}), 2.24(\mathrm{dd}, 1 \mathrm{H}, J=12.8,18.8 \mathrm{~Hz})$, $2.10(\mathrm{~s}, \mathrm{H}), 1.72(\mathrm{ddd}, 1 \mathrm{H}, J=8.8,11.6,12.8 \mathrm{~Hz}), 0.377$ (s, 3H), 0.362 (s, 3H)

${ }^{13} \mathbf{C} \mathbf{N M R}\left(\mathbf{C D C l}_{3}, \mathbf{1 0 0} \mathbf{M H z}\right) \delta$ 216.2, 205.8, 201.3, 135.7, 133.8, 133.8, 129.8, 128.1, 128.1, 55.9, 46.6, 41.8, $38.6,29.7,22.7,-4.51,-4.72$

HRMS (ESI): $[\mathrm{M}+\mathrm{Na}]^{+}$calcd for $\mathrm{C}_{17} \mathrm{H}_{22} \mathrm{O}_{3} \mathrm{SiNa}$ : 325.1230, found: 325.1234

IR(neat) $v 2956,1741,1718,1427,1366,1253,1166,1114,820,738,703,650,533 \mathrm{~cm}^{-1}$

$[\alpha]_{\mathrm{D}}{ }^{26}-115\left(c 4.0, \mathrm{CHCl}_{3}\right)$

$\mathbf{R}_{\mathbf{f}}(n$-Hexane: EtOAc $=2: 1$, color reagent: Hanessian's stain reagent $): 0.65$ 


\subsection{Dimethyl acetal protection of compound 9}

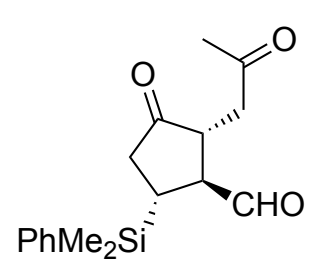

9

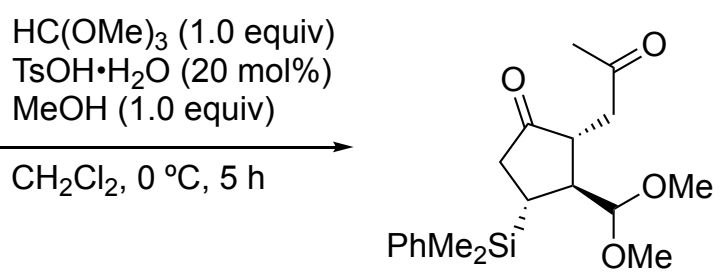

4

To a solution of aldehyde $9(30.2 \mathrm{mg}, 0.10 \mathrm{mmol}), \mathrm{HC}(\mathrm{OMe})_{3}(10.9 \mu \mathrm{L}, 0.10 \mathrm{mmol})$ and $\mathrm{MeOH}(4.1 \mu \mathrm{L}$, $0.10 \mathrm{mmol})$ in $\mathrm{CH}_{2} \mathrm{Cl}_{2}(250 \mu \mathrm{L}), \mathrm{TsOH} \cdot \mathrm{H}_{2} \mathrm{O}(3.6 \mathrm{mg}, 0.020 \mathrm{mmol})$ was added at $0{ }^{\circ} \mathrm{C}$. After stirring the reaction mixture at this temperature for $5 \mathrm{~h}$, the reaction mixture was quenched by the addition of sat. aq. $\mathrm{NaHCO}_{3}(5$ $\mathrm{mL})$. Upon completion, $\mathrm{H}_{2} \mathrm{O}(2 \mathrm{~mL})$ was added and the mixture was extracted with EtOAc $(3 \times 5 \mathrm{~mL})$. The combined organic extracts were washed with sat. aq. $\mathrm{NaHCO}_{3}(5 \mathrm{~mL})$ and sat. aq. $\mathrm{NaCl}$ solution $(5 \mathrm{~mL})$. Then, the combined organic extracts were dried with anhydrous $\mathrm{Na}_{2} \mathrm{SO}_{4}$, filtered, and concentrated under reduced pressure. After concentration in vacuo, the reaction mixture was purified by column chromatography on silica gel ( $n$-Hexane: EtOAc $=6: 1)$ to give the target compound 4 in $85 \%$ yield $(29.6 \mathrm{mg}, 0.085 \mathrm{mmol})$.

(2R,3S,4R)-3-(Dimethoxymethyl)-4-(dimethyl(phenyl)silyl)-2-(2-oxopropyl)cyclopentan-1-one (4)<smiles>COC(OC)[C@@H]1[C@H](CC(C)=O)C(=O)C[C@H]1[SiH2]</smiles>

Yield: $85 \%(29.6 \mathrm{mg})$

Physical State: Yellow oil

${ }^{1} \mathbf{H}$ NMR $\left(\mathbf{C D C l}_{3}, \mathbf{4 0 0} \mathbf{M H z}\right) \delta$ 7.52-7.54 (m, 2H), 7.35-7.39 (m, 3H), 3.85 (d, 1H, J= 3.2 Hz), 3.15 (s, 3H), $3.13(\mathrm{~s}, 3 \mathrm{H}), 2.86(\mathrm{ddd}, 2 \mathrm{H}, J=4.4,18.4,19.2 \mathrm{~Hz}), 2.51(\mathrm{dq}, 1 \mathrm{H}, J=1.2,8.8 \mathrm{~Hz}), 2.40(\mathrm{ddd}, 1 \mathrm{H}, J=1.6,9.6$, $16.8 \mathrm{~Hz}), 2.34(\mathrm{dd}, 1 \mathrm{H}, J=3.2,11.6 \mathrm{~Hz}), 2.28(\mathrm{dd}, 1 \mathrm{H}, J=12.8,18.8 \mathrm{~Hz}), 2.07(\mathrm{~s}, 3 \mathrm{H}), 1.50(\mathrm{ddd}, 1 \mathrm{H}, J=$ 9.2, 11.2, $12.8 \mathrm{~Hz}), 0.401(\mathrm{~s}, 3 \mathrm{H}), 0.330(\mathrm{~s}, 3 \mathrm{H})$

${ }^{13} \mathbf{C} \mathbf{N M R}\left(\mathbf{C D C l}_{3}, \mathbf{1 0 0} \mathrm{MHz}\right) \delta$ 219.7, 207.0, 138.3, 133.7, 133.7, 129.2, 128.0, 128.0, 107.6, 56.5, 56.1, 46.2, $45.5,43.3,39.9,29.8,22.0,-3.48,-5.40$

HRMS (ESI): $[\mathrm{M}+\mathrm{Na}]^{+}$calcd for $\mathrm{C}_{19} \mathrm{H}_{28} \mathrm{O}_{4} \mathrm{SiNa}$ : 371.1649, found: 371.1651

IR(neat)v1738, 1714, 1366, 1252, 1073, 951, 820, 737, $702 \mathrm{~cm}^{-1}$

$[\alpha]_{\mathrm{D}}{ }^{26}-35.6\left(c 2.95, \mathrm{CHCl}_{3}\right)$

$\mathbf{R}_{\mathbf{f}}(n$-Hexane: EtOAc $=2: 1$, color reagent: Hanessian's stain reagent): 0.63

The enantiomeric ratio was determined by HPLC using CHIRALPACK ${ }^{\circledR}$ ID ( $n$-Hexane / $i$-PrOH $=10: 1$; flow rate $1.0 \mathrm{ml} / \mathrm{min}$, minor isomer $t_{R}=9.27 \mathrm{~min}$, major isomer $\left.t_{R}=10.7 \mathrm{~min}\right)(98 \%$ ee $)$. 


\subsection{Asymmetric domino Michael/Michael reaction between ketone 8 and aldehyde $6 \mathrm{~b}$}

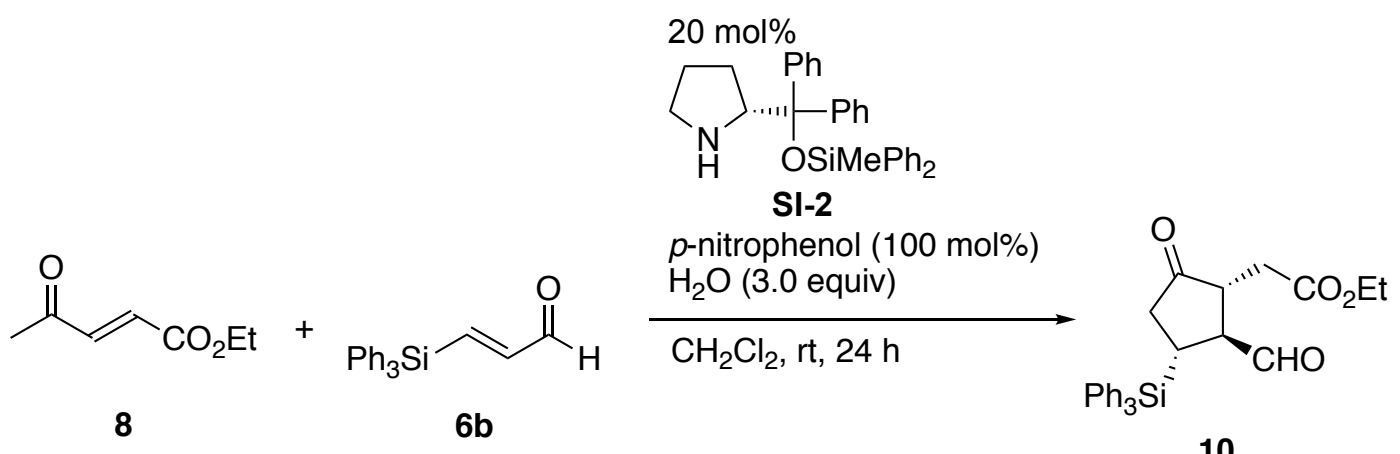

To a solution of ketone $\mathbf{8}^{[2]}(284 \mathrm{mg}, 2.0 \mathrm{mmol})$, aldehyde $\mathbf{6 b}(314 \mathrm{mg}, 1.0 \mathrm{mmol}), \mathrm{H}_{2} \mathrm{O}(540 \mu \mathrm{L}, 3.0 \mathrm{mmol})$ and $p$-nitrophenol $(139 \mathrm{mg}, 1.0 \mathrm{mmol})$ in $\mathrm{CH}_{2} \mathrm{Cl}_{2}(250 \mu \mathrm{L})$, organocatalyst $\mathbf{S I - 2}(90.0 \mathrm{mg}, 0.20 \mathrm{mmol})$ was added at room temperature. After stirring the reaction mixture at this temperature for $24 \mathrm{~h}$, the reaction mixture was directly purified by column chromatography on silica gel ( $n$-Hexane: EtOAc $=6: 1)$ to give the target compound 10 (415 mg, $0.91 \mathrm{mmol}$ ) in $91 \%$ yield (single isomer).

Ethyl 2-((1R,2S,3R)-2-formyl-5-oxo-3-(triphenylsilyl)cyclopentyl)acetate (10)<smiles>CCOC(=O)C[C@H]1C(=O)C[C@@H]([SiH3])[C@H]1C=O</smiles>

Yield: $91 \%(415 \mathrm{mg})$

Physical State: Yellow oil

${ }^{1} \mathbf{H}$ NMR $\left(\mathbf{C D C l}_{3}, \mathbf{4 0 0} \mathbf{M H z}\right) \delta 9.27(\mathrm{~d}, 1 \mathrm{H}, J=2.0 \mathrm{~Hz}), 7.57-7.59(\mathrm{~m}, 6 \mathrm{H}), 7.38-7.48(\mathrm{~m}, 9 \mathrm{H})$, 3.98-4.08 (m, $2 \mathrm{H}), 3.15(\mathrm{dt}, 1 \mathrm{H}, J=3.2,7.2 \mathrm{~Hz}), 2.73-2.81(\mathrm{~m}, 2 \mathrm{H}), 2.54(\mathrm{dd}, 1 \mathrm{H}, J=4.4,17.6 \mathrm{~Hz}), 2.39-2.48(\mathrm{~m}, 3 \mathrm{H}), 1.17$ $(\mathrm{t}, 3 \mathrm{H}, J=11.2 \mathrm{~Hz})$

${ }^{13} \mathbf{C}$ NMR (CDCl ${ }_{3}, \mathbf{1 0 0}$ MHz) $\delta$ 215.0, 200.6, 171.2, 136.0, 136.0, 136.0, 136.0, 136.0, 136.0, 132.2, 132.2, $132.2,130.2,130.2,130.2,128.3,128.3,128.3,128.3,128.3,128.3,60.9,55.8,47.1,39.7,32.5,21.8,14.0$ HRMS (ESI): $[\mathrm{M}+\mathrm{Na}]^{+}$calcd for $\mathrm{C}_{28} \mathrm{H}_{28} \mathrm{O}_{4} \mathrm{SiNa}$ : 479.1649, found: 479.1649

IR(neat) $v 2726,1729,1428,1192,1110,741,702,515 \mathrm{~cm}^{-1}$

$[\alpha]_{\mathrm{D}}{ }^{26}-93.9\left(c 0.50, \mathrm{CHCl}_{3}\right)$

$\mathbf{R}_{\mathbf{f}}(n$-Hexane: EtOAc $=2: 1$, color reagent: Hanessian's stain reagent $): 0.50$ 


\subsection{Dimethyl acetal protection of compound 10}

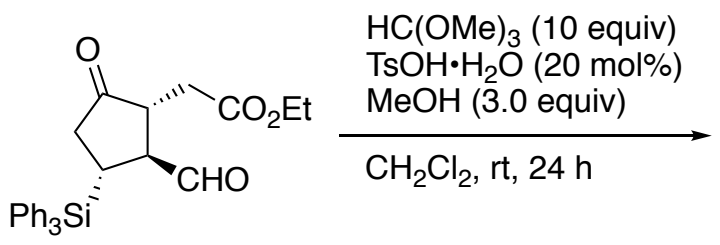

10

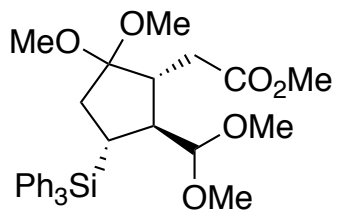

12

To a solution of aldehyde $10(45.6 \mathrm{mg}, 0.10 \mathrm{mmol}), \mathrm{HC}(\mathrm{OMe})_{3}(109 \mu \mathrm{L}, 1.0 \mathrm{mmol})$ and $\mathrm{MeOH}(12 \mu \mathrm{L}$, $0.30 \mathrm{mmol})$ in $\mathrm{CH}_{2} \mathrm{Cl}_{2}(250 \mu \mathrm{L})$, TsOH$\cdot \mathrm{H}_{2} \mathrm{O}(3.8 \mathrm{mg}, 0.020 \mathrm{mmol})$ was added at room temperature. After stirring the reaction mixture at this temperature for $24 \mathrm{~h}$, the reaction mixture was quenched by the addition of sat. aq. $\mathrm{NaHCO}_{3}(5 \mathrm{~mL})$. Upon completion, $\mathrm{H}_{2} \mathrm{O}(2 \mathrm{~mL})$ was added and the mixture was extracted with EtOAc $(3 \times 5 \mathrm{~mL})$. The combined organic extracts were washed with sat. aq. $\mathrm{NaHCO}_{3}(5 \mathrm{~mL})$ and sat. aq. $\mathrm{NaCl}$ solution $(5 \mathrm{~mL})$. Then, the combined organic extracts were dried with anhydrous $\mathrm{Na}_{2} \mathrm{SO}_{4}$, filtered, and concentrated under reduced pressure. After concentration in vacuo, the reaction mixture was purified by column chromatography on silica gel $(n$-Hexane: EtOAc $=6: 1)$ to give the target compound 12 in $85 \%$ yield (45.5 mg, $0.085 \mathrm{mmol})$.

Methyl 2-((1R,4R,5S)-5-(dimethoxymethyl)-2,2-dimethoxy-4-(triphenylsilyl)cyclopentyl)acetate (12)<smiles>COC(OC)[C@H]1[C@@H](CC(=O)O)CC(OC)(OC)[C@@H]1[SiH3]</smiles>

Yield: $85 \%(45.5 \mathrm{mg})$

Physical State: Yellow oil

${ }^{1}$ H NMR $\left(\mathbf{C D C l}_{3}, \mathbf{4 0 0} \mathbf{M H z}\right) \delta$ 7.59-7.71 (m, 5H), 7.33-7.43 (m, 10H), 3.99 (dd, 1H, J = 3.6, 11.2 Hz), 3.51 $(\mathrm{s}, 3 \mathrm{H}), 3.21(\mathrm{~s}, 3 \mathrm{H}), 3.12(\mathrm{~s}, 3 \mathrm{H}), 3.08(\mathrm{~s}, 3 \mathrm{H}), 2.93(\mathrm{~s}, 3 \mathrm{H}), 2.55-2.77(\mathrm{~m}, 3 \mathrm{H}), 2.31-2.45(\mathrm{~m}, 1 \mathrm{H}), 2.07-2.20$ $(\mathrm{m}, 2 \mathrm{H}), 1.95(\mathrm{ddd}, 1 \mathrm{H}, J=5.6,15.6,21.2 \mathrm{~Hz})$

${ }^{13} \mathbf{C}$ NMR (CDCl ${ }_{3}, \mathbf{1 0 0}$ MHz) $\delta 172.4,136.0,136.0,136.0,136.0,136.0,136.0,133.7,133.7,133.7,129.8$, $129.8,129.8,128.1,128.1$, 128.1 , 128.1 , 128.1 , 128.1 , 107.4, 106.5, 56.0, 55.8, 51.4, 49.0, 48.2 , 46.0, 45.7, $41.1,33.7,19.4$

HRMS (ESI): $[\mathrm{M}+\mathrm{Na}]^{+}$calcd for $\mathrm{C}_{31} \mathrm{H}_{38} \mathrm{O}_{6} \mathrm{SiNa}$ : 557.2330, found: 557.2330

IR(neat) $v 1739,1428,1197,1110,1073,741,702,513 \mathrm{~cm}^{-1}$

$[\alpha]_{\mathrm{D}}{ }^{26}-43.5\left(c 1.9, \mathrm{CHCl}_{3}\right)$

$\mathbf{R}_{\mathbf{f}}(n$-Hexane: EtOAc $=2: 1$, color reagent: Hanessian's stain reagent $): 0.65$ 


\subsection{Deprotection of compound 12}

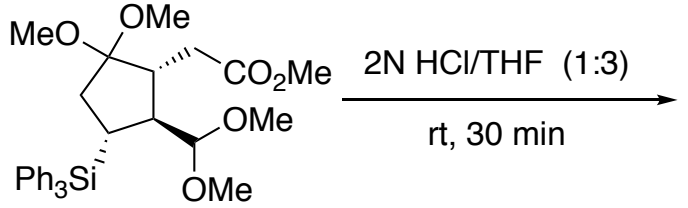

12

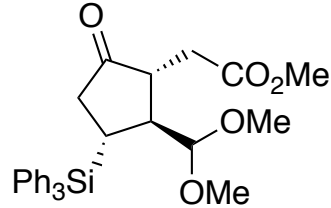

13

To a solution of ketal $12(53.4 \mathrm{mg}, 0.10 \mathrm{mmol})$ in THF $(300 \mu \mathrm{L}), 2 \mathrm{~N} \mathrm{HCl}(100 \mu \mathrm{L})$ was added at room temperature. After stirring the reaction mixture at this temperature for $30 \mathrm{~min}$, the reaction mixture was quenched by the addition of sat. aq. $\mathrm{NaHCO}_{3}(5 \mathrm{~mL})$. Upon completion, $\mathrm{H}_{2} \mathrm{O}(2 \mathrm{~mL})$ was added and the mixture was extracted with EtOAc $(3 \times 5 \mathrm{~mL})$. The combined organic extracts were washed with sat. aq. $\mathrm{NaHCO}_{3}(5 \mathrm{~mL})$ and sat. aq. $\mathrm{NaCl}$ solution $(5 \mathrm{~mL})$. Then, the combined organic extracts were dried with anhydrous $\mathrm{Na}_{2} \mathrm{SO}_{4}$, filtered, and concentrated under reduced pressure. After concentration in vacuo, the reaction mixture was purified by column chromatography on silica gel $(n$-Hexane: EtOAc $=6: 1)$ to give the target compound 13 in $79 \%$ yield (38.5 mg, $0.079 \mathrm{mmol}$ ).

Methyl 2-((1R,2S,3R)-2-(dimethoxymethyl)-5-oxo-3-(triphenylsilyl)cyclopentyl)acetate (13)<smiles>COC(OC)[C@H]1[C@@H](CC(C)=O)CC(=O)[C@@H]1[SiH2]c1ccccc1</smiles>

Yield: $79 \%(38.5 \mathrm{mg})$

Physical State: Colorless oil

${ }^{1} \mathrm{H}$ NMR $\left(\mathrm{CDCl}_{3}, \mathbf{4 0 0} \mathrm{MHz}\right)$ d 7.62-7.63 (m, 5H), 7.35-7.45 (m, 10H), $3.90(\mathrm{~d}, 1 \mathrm{H}, J=2.4 \mathrm{~Hz}), 3.53(\mathrm{~s}, 3 \mathrm{H})$, $3.19(\mathrm{~s}, 3 \mathrm{H}), 2.94(\mathrm{~s}, 3 \mathrm{H}), 2.72(\mathrm{dd}, 1 \mathrm{H}, J=5.6,11.2 \mathrm{~Hz}), 2.54-2.65(\mathrm{~m}, 4 \mathrm{H}), 2.27-2.40(\mathrm{~m}, 2 \mathrm{H})$

${ }^{13} \mathbf{C}$ NMR $\left(\mathbf{C D C l}_{3}, \mathbf{1 0 0} \mathrm{MHz}\right) \delta 218.4,172.4,136.0,136.0,136.0,136.0,136.0,136.0,133.7,133.7,133.7$, $129.8,129.8,129.8,128.1,128.1,128.1,128.1,128.1,128.1$, 106.5, 56.0, 55.8, 51.4, 46.0, 45.7, 41.1, 33.7, 19.4

HRMS (ESI): $[\mathrm{M}+\mathrm{Na}]^{+}$calcd for $\mathrm{C}_{29} \mathrm{H}_{32} \mathrm{O}_{5} \mathrm{SiNa}$ : 511.1911, found: 511.1911

IR(neat) $v 1738,1428,1178,1109,1074,702,512,413 \mathrm{~cm}^{-1}$

$[\alpha]_{\mathrm{D}}{ }^{26}-106\left(c 0.60, \mathrm{CHCl}_{3}\right)$

$\mathbf{R}_{\mathbf{f}}(n$-Hexane: EtOAc $=2: 1$, color reagent: Hanessian's stain reagent $): 0.60$

The enantiomeric ratio was determined by HPLC using CHIRALPACK ${ }^{\circledR} \mathrm{IC}(n$-Hexane $/ i$-PrOH $=10: 1$; flow rate $1.0 \mathrm{ml} / \mathrm{min}$, minor isomer $\mathrm{t}_{\mathrm{R}}=22.4 \mathrm{~min}$, major isomer $\left.\mathrm{t}_{\mathrm{R}}=18.1 \mathrm{~min}\right)(96 \%$ ee $)$. 


\subsection{One-pot procedure to synthesize compound 13}

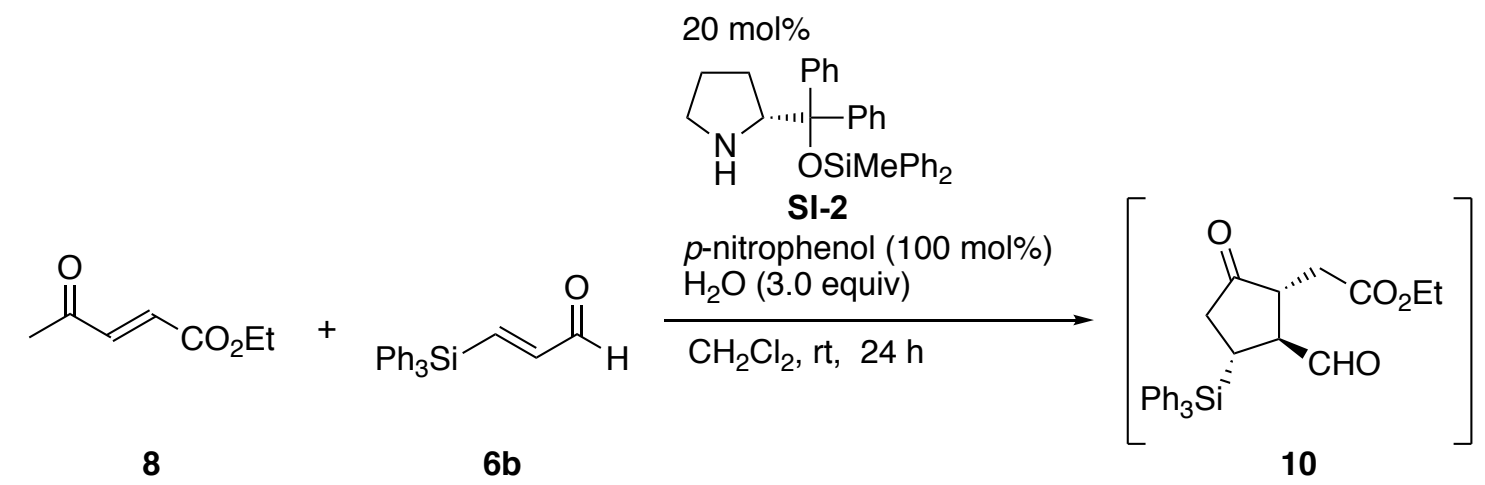

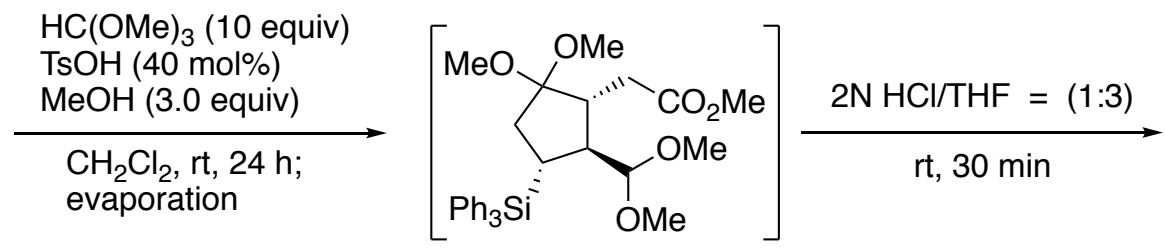

12<smiles>COC(OC)[C@@H]1[C@H](CC(C)=O)C(=O)C[C@H]1[SiH3]</smiles>

13

To a solution of ketone $\mathbf{8}(284 \mathrm{mg}, 2.0 \mathrm{mmol})$, aldehyde $\mathbf{6 b}(314 \mathrm{mg}, 1.0 \mathrm{mmol}), \mathrm{H}_{2} \mathrm{O}(540 \mu \mathrm{L}, 3.0 \mathrm{mmol})$ and $p$-nitrophenol $(139 \mathrm{mg}, 1.0 \mathrm{mmol})$ in $\mathrm{CH}_{2} \mathrm{Cl}_{2}(250 \mu \mathrm{L})$, organocatalyst $\mathbf{S I - 2}(90.0 \mathrm{mg}, 0.20 \mathrm{mmol})$ was added at room temperature. After stirring the reaction mixture at this temperature for $24 \mathrm{~h}, \mathrm{HC}(\mathrm{OMe})_{3}(1.09$ $\mathrm{mL}, 10 \mathrm{mmol})$ and $\mathrm{MeOH}(120 \mu \mathrm{L}, 3.0 \mathrm{mmol}), \mathrm{TsOH} \cdot \mathrm{H}_{2} \mathrm{O}(38 \mathrm{mg}, 0.20 \mathrm{mmol})$ was added at room temperature. After stirring the reaction mixture at this temperature for $24 \mathrm{~h}$, the reaction mixture was concentrated under reduced pressure. To a residue in THF $(3.0 \mathrm{~mL}), 2 \mathrm{~N} \mathrm{HCl}(1.0 \mathrm{~mL})$ was added at room temperature. After stirring the reaction mixture at this temperature for $30 \mathrm{~min}$, the reaction mixture was quenched by the addition of sat. aq. $\mathrm{NaHCO}_{3}(50 \mathrm{~mL})$. Upon completion, $\mathrm{H}_{2} \mathrm{O}(20 \mathrm{~mL})$ was added and the mixture was extracted with EtOAc $(3 \times 50 \mathrm{~mL})$. The combined organic extracts were washed with sat. aq. $\mathrm{NaHCO}_{3}(50 \mathrm{~mL})$ and sat. aq. $\mathrm{NaCl}$ solution $(50 \mathrm{~mL})$. Then, the combined organic extracts were dried with anhydrous $\mathrm{Na}_{2} \mathrm{SO}_{4}$, filtered, and concentrated under reduced pressure. After concentration in vacuo, the reaction mixture was purified by column chromatography on silica gel ( $n$-Hexane: EtOAc $=6: 1)$ to give the target compound 13 in $60 \%$ yield (265 mg, $0.60 \mathrm{mmol})$. 


\subsection{One-pot synthesis of compound $3 \mathrm{~b}$ from compound 13}

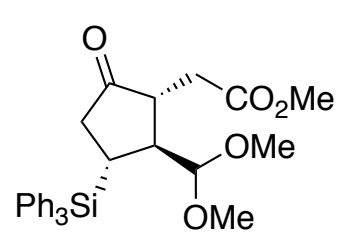

13

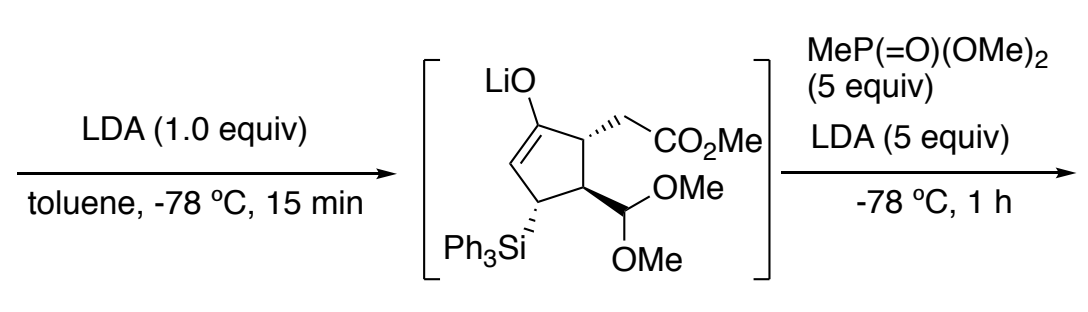

14

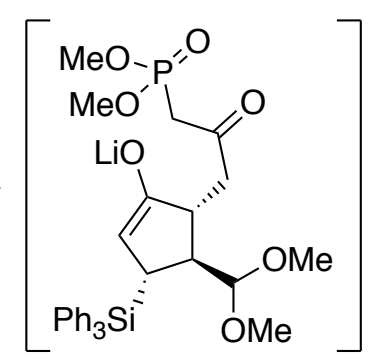

15

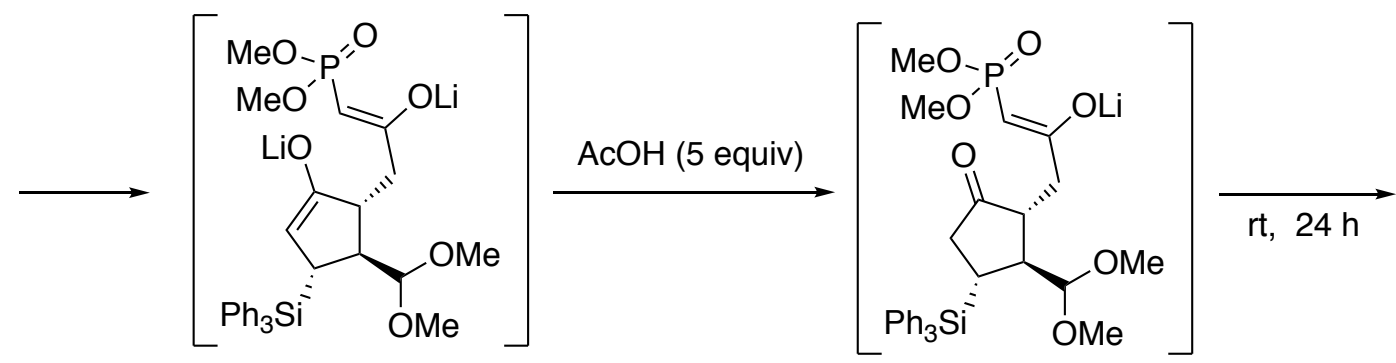

16

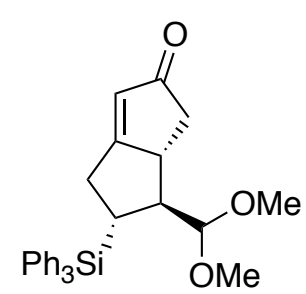

3b

To a solution of compound $13(97.6 \mathrm{mg}, 0.20 \mathrm{mmol})$ in toluene $(2.0 \mathrm{~mL})$, LDA (200 $\mu \mathrm{L}, 1.0 \mathrm{M}$ in THF) was added at $-78{ }^{\circ} \mathrm{C}$. After stirring the reaction mixture at this temperature for $15 \mathrm{~min}$, to a reaction mixture, dimethyl methylphosphonate $(124 \mathrm{mg}, 1.0 \mathrm{mmol})$ and LDA $\left(1.0 \mathrm{~mL}, 1.0 \mathrm{M}\right.$ in THF) were added at $-78^{\circ} \mathrm{C}$. After stirring the reaction mixture at this temperature for $1 \mathrm{~h}$, to a reaction mixture, actetic acid (60 mg, 1.0 $\mathrm{mmol})$ in toluene $(3.0 \mathrm{~mL})$ was added at $-78{ }^{\circ} \mathrm{C}$. After stirring the reaction mixture at room temperature for 24 $\mathrm{h}, \mathrm{H}_{2} \mathrm{O}(10 \mathrm{~mL})$ was added and the mixture was extracted with EtOAc $(3 \times 10 \mathrm{~mL})$. The combined organic extracts were washed with sat. aq. $\mathrm{NaCl}$ solution $(40 \mathrm{~mL})$. Then, the combined organic extracts were dried with anhydrous $\mathrm{Na}_{2} \mathrm{SO}_{4}$, filtered, and concentrated under reduced pressure. After concentration in vacuo, the reaction mixture was purified by column chromatography on silica gel ( $n$-Hexane: EtOAc $=3: 1)$ to give the target compound $\mathbf{3 b}$ in $86 \%$ yield $(78.0 \mathrm{mg}, 0.17 \mathrm{mmol})$.

(5R,6S,6aR)-6-(Dimethoxymethyl)-5-(triphenylsilyl)-4,5,6,6a-tetrahydropentalen-2(1H)-one (3b)<smiles>COC(OC)[C@@H]1[C@H]([SiH3])CC2=CC(=O)C[C@@H]21</smiles>

Yield: $86 \%(78.0 \mathrm{mg})$

Physical State: White amorphous

${ }^{1} \mathbf{H}$ NMR $\left(\mathbf{C D C l}_{3}, 400 \mathrm{MHz}\right) \delta$ 7.56-7.58 (m, 6H), 7.34-7.44 (m, 9H), $5.68(\mathrm{dt}, 1 \mathrm{H}, J=0.80,2.0 \mathrm{~Hz}), 3.67(\mathrm{~d}$, $1 \mathrm{H}, J=2.8 \mathrm{~Hz}), 3.22-3.25(\mathrm{~m}, 1 \mathrm{H}), 3.22(\mathrm{~s}, 3 \mathrm{H}), 3.09(\mathrm{dd}, 1 \mathrm{H}, J=12.8,18.0 \mathrm{~Hz}), 2.99(\mathrm{~s}, 3 \mathrm{H}), 2.75(\mathrm{dd}, 1 \mathrm{H}$, $J=6.8,18.0 \mathrm{~Hz}), 2.62(\mathrm{dd}, 1 \mathrm{H}, J=6.4,18.8 \mathrm{~Hz}), 2.57(\mathrm{ddd}, 1 \mathrm{H}, J=6.8,10.4,12.4 \mathrm{~Hz}), 2.05-2.11(\mathrm{~m}, 2 \mathrm{H})$

${ }^{13} \mathbf{C}$ NMR (CDCl ${ }_{3}, \mathbf{1 0 0}$ MHz) $\delta 211.3,189.6,135.8,135.8,135.8,135.8,135.8,135.8,133.7,133.7,133.7$, 
$129.7,129.7,129.7,128.0,128.0,128.0,128.0,128.0,128.0,124.9,106.4,56.4,56.1,49.5,46.3,43.2,30.2$, 24.4

HRMS (ESI): $[\mathrm{M}+\mathrm{Na}]^{+}$calcd for $\mathrm{C}_{29} \mathrm{H}_{30} \mathrm{O}_{3} \mathrm{SiNa}$ : 477.1856, found: 477.1859

IR(neat) $v 1701,1628,1428,1189,1110,1074,741,702,504 \mathrm{~cm}^{-1}$

$[\alpha]_{\mathrm{D}}{ }^{26}-85.9\left(c 10.0, \mathrm{CHCl}_{3}\right)$

$\mathbf{R}_{\mathbf{f}}(n$-Hexane: EtOAc $=2: 1$, color reagent: Hanessian's stain reagent $): 0.60$

\subsection{One-pot synthesis of compound 19 from compound $3 b$}

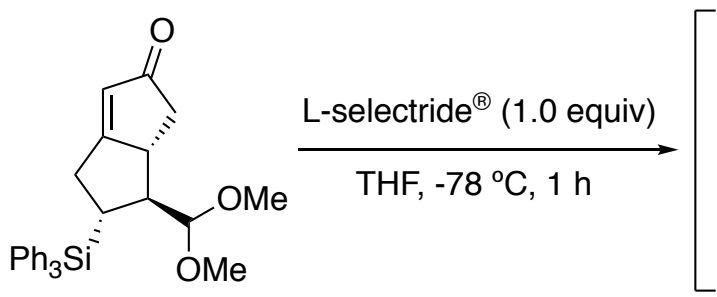

$3 \mathbf{b}$

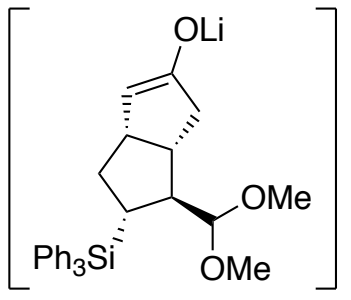

18

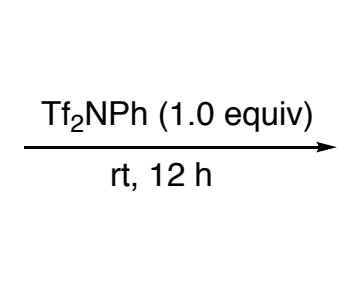

$\mathrm{h}_{3} \mathrm{Si}$

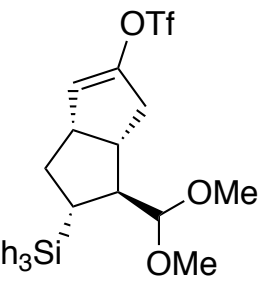

19

To a solution of compound $3 \mathbf{b}(45.4 \mathrm{mg}, 0.10 \mathrm{mmol})$ in THF $(1.0 \mathrm{~mL})$, L-selectride ${ }^{\circledR}(120 \mu \mathrm{L}, 1.0 \mathrm{M}$ in THF) was added at $-78{ }^{\circ} \mathrm{C}$. After stirring the reaction mixture at this temperature for $1 \mathrm{~h}$, to a reaction mixture, $\mathrm{Tf}_{2} \mathrm{NPh}\left(43 \mathrm{mg}, 0.10 \mathrm{mmol}\right.$ ) was added at $-78{ }^{\circ} \mathrm{C}$. After stirring the reaction mixture at this temperature for 1 $\mathrm{h}$, the reaction was quenched by the addition of buffer $(1.0 \mathrm{~mL})$ and extracted with EtOAc $(3 \times 5 \mathrm{~mL})$. The combined organic extracts were washed with sat. aq. $\mathrm{NaCl}$ solution $(10 \mathrm{~mL})$. Then, the combined organic extracts were dried with anhydrous $\mathrm{Na}_{2} \mathrm{SO}_{4}$, filtered, and concentrated under reduced pressure. After concentration in vacuo, the reaction mixture was purified by column chromatography on silica gel ( $n$-Hexane: EtOAc $=20: 1)$ to give the target compound 19 in $71 \%$ yield $(41.7 \mathrm{mg}, 0.071 \mathrm{mmol})$.

\section{(3aS,5R,6S)-6-(Dimethoxymethyl)-5-(triphenylsilyl)-1,3a,4,5,6,6a-hexahydropentalen-2-yl}

\section{trifluoromethanesulfonate (19)}

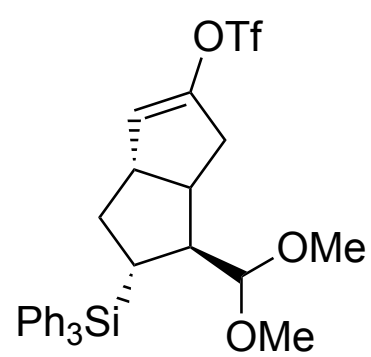

Yield: $71 \%(41.9 \mathrm{mg})$

Physical State: White amorphous

${ }^{1} \mathbf{H}$ NMR $\left(\mathbf{C D C l}_{3}, 400 \mathrm{MHz}\right) \delta$ 7.59-7.62 (m, 6H), 7.37-7.43 (m, 9H), $5.47(\mathrm{~d}, 1 \mathrm{H}, J=2.0), 3.90(\mathrm{~d}, 1 \mathrm{H}, J=$ $2.4 \mathrm{~Hz}), 3.16(\mathrm{~s}, 3 \mathrm{H}), 3.09-3.19(\mathrm{~m}, 1 \mathrm{H}), 2.95(\mathrm{~s}, 3 \mathrm{H}), 2.85-2.96(\mathrm{~m}, 1 \mathrm{H}), 2.76(\mathrm{tdd}, 1 \mathrm{H}, J=2.0,9.2,16.8 \mathrm{~Hz})$, 2.25-2.37 (m, 2H), 1.95-2.06 (m, 1H), $2.01(\mathrm{dd}, 1 \mathrm{H}, J=6.4,11.6 \mathrm{~Hz}), 1.37(\mathrm{dt}, 1 \mathrm{H}, J=8.4,12.8 \mathrm{~Hz})$

${ }^{13} \mathbf{C} \mathbf{N M R}\left(\mathbf{C D C l}_{3}, \mathbf{1 0 0} \mathbf{M H z}\right) \delta 146.8,135.9,135.9,135.9,135.9,135.9,135.9,134.4,134.4,134.4,129.5$, $129.5,129.5$, 127.9, 127.9, 127.9, 127.9, 127.9, 127.9, 127.7, 121.2, 106.7, 55.8, 55.4, 53.1 , 46.0 , 40.2, 37.7, 
$36.7,25.5$

HRMS (ESI): [M+Na] ${ }^{+}$calcd for $\mathrm{C}_{30} \mathrm{H}_{31} \mathrm{~F}_{3} \mathrm{O}_{5} \mathrm{SSiNa}$ : 611.1505, found: 611.1510

$\operatorname{IR}\left(\right.$ neat) $v 1422,1241,1213,1142,1108,1082,907,741,702,610,516 \mathrm{~cm}^{-1}$

$[\alpha]_{\mathrm{D}}{ }^{26}-50.0\left(c 1.6, \mathrm{CHCl}_{3}\right)$

$\mathbf{R}_{\mathbf{f}}(n$-Hexane: EtOAc $=10: 1$, color reagent: Hanessian's stain reagent $): 0.70$

\subsection{Suzuki-Miyaura reaction of compound 19}

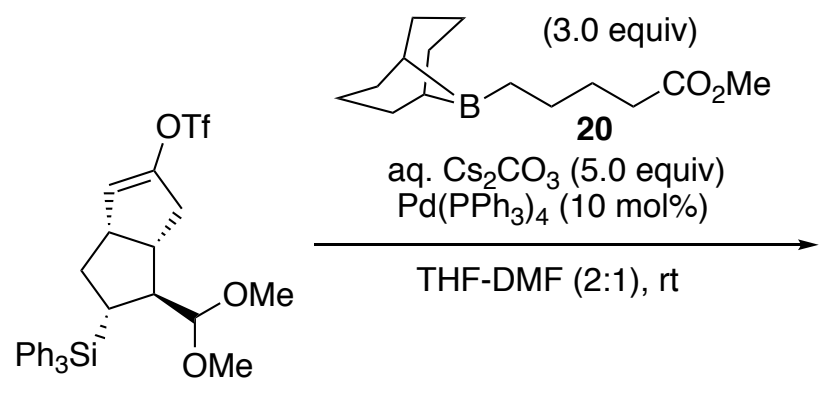

19

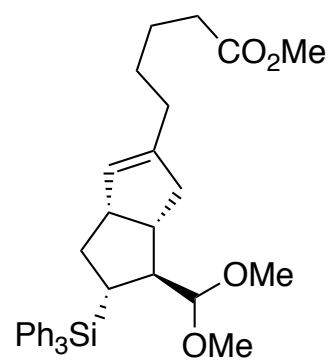

21

To a solution of methyl pent-4-enoate ( $52 \mathrm{mg}, 0.46 \mathrm{mmol})$ in THF (2 mL), 9-BBN (3.0 mL, $0.5 \mathrm{M}$ in THF) was added at room temperature. After stirring the reaction mixture at this temperature for $1 \mathrm{~h}$, to a reaction mixture, aq. $\mathrm{Cs}_{2} \mathrm{CO}_{3}\left(91 \mu \mathrm{L}, 3.0 \mathrm{M}\right.$ in $\left.\mathrm{H}_{2} \mathrm{O}, 2.73 \mathrm{mmol}\right)$ was added. Then, to a reaction micture, compound 19 (270 mg, $0.46 \mathrm{mmol})$ in DMF (2 mL) and $\mathrm{Pd}\left(\mathrm{PPh}_{3}\right)_{4}(79 \mathrm{mg}, 0.068 \mathrm{mmol})$ in THF $(2.0 \mathrm{~mL})$ were added at room temperature. After stirring the reaction mixture at this temperature for $2 \mathrm{~h}$, the reaction was quenched by the addition of water $(5.0 \mathrm{~mL})$ and extracted with EtOAc $(3 \times 20 \mathrm{~mL})$. The combined organic extracts were washed with sat. aq. $\mathrm{NaCl}$ solution $(60 \mathrm{~mL})$. Then, the combined organic extracts were dried with anhydrous $\mathrm{Na}_{2} \mathrm{SO}_{4}$, filtered, and concentrated under reduced pressure. After concentration in vacuo, the reaction mixture was purified by column chromatography on silica gel $(n$-Hexane: EtOAc $=12: 1)$ to give the target compound 21 in $82 \%$ yield (209 $\mathrm{mg}, 0.38 \mathrm{mmol})$.

\section{Methyl 5-((3aS,5R,6S)-6-(dimethoxymethyl)-5-(triphenylsilyl)-1,3a,4,5,6,6a-hexahydropentalen-2-} yl)pentanoate (21)<smiles>COC(=O)CCCCC1=C[C@H]2CC([SiH2]c3ccccc3)[C@@H](C(OC)OC)[C@H]2C1</smiles>

Yield: $82 \%(209 \mathrm{mg})$

Physical State: Colorless oil

${ }^{1} \mathbf{H}$ NMR $\left(\mathbf{C D C l}_{3}, \mathbf{4 0 0} \mathbf{M H z}\right)$ d 7.59-7.61 (m, 5H), 7.32-7.40 (m, 10H), $5.14(\mathrm{~s}, 1 \mathrm{H}), 3.90(\mathrm{~d}, 1 \mathrm{H}, J=3.2 \mathrm{~Hz})$, 
$3.66(\mathrm{~s}, 3 \mathrm{H}), 3.15(\mathrm{~s}, 3 \mathrm{H}), 3.07-3.12(\mathrm{~m}, 1 \mathrm{H}), 2.84(\mathrm{ddd}, 1 \mathrm{H}, J=3.6,9.2,18.0 \mathrm{~Hz}), 2.29(\mathrm{t}, 2 \mathrm{H}, J=7.6 \mathrm{~Hz})$, $1.63-1.70(\mathrm{~m}, 3 \mathrm{H}), 1.80-2.00(\mathrm{~m}, 6 \mathrm{H}), 1.29-1.58(\mathrm{~m}, 6 \mathrm{H})$

${ }^{13} \mathbf{C}$ NMR $\left(\mathbf{C D C l}_{3}, \mathbf{1 0 0} \mathbf{M H z}\right) \delta 174.1,141.6,135.9,135.9,135.9,135.9,135.9,135.9,135.8,135.1,135.1$, 135.1 129.0, 129.0, 129.0, 127.6, 127.6, 127.6, 127.6, 127.6, 127.6, 107.6, 70.8, 55.4, 51.5, 43.5, 41.6, 37.3, $33.8,31.9,30.4,27.1,26.1,24.5,21.9$

HRMS (ESI): [M+Na] $]^{+}$calcd for $\mathrm{C}_{35} \mathrm{H}_{42} \mathrm{O}_{4} \mathrm{SiNa}: 577.2744$, found: 577.2740

IR(neat) $v 1739,1428,1108,741,702,515 \mathrm{~cm}^{-1}$

$[\alpha]_{\mathrm{D}}{ }^{26}-61.2\left(c 0.50, \mathrm{CHCl}_{3}\right)$

$\mathbf{R}_{\mathbf{f}}(n$-Hexane: EtOAc $=3: 1$, color reagent: Hanessian's stain reagent $): 0.50$

\subsection{Tamao-Fleming oxidation of compound 21}

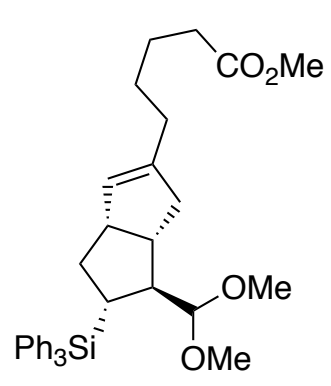

21

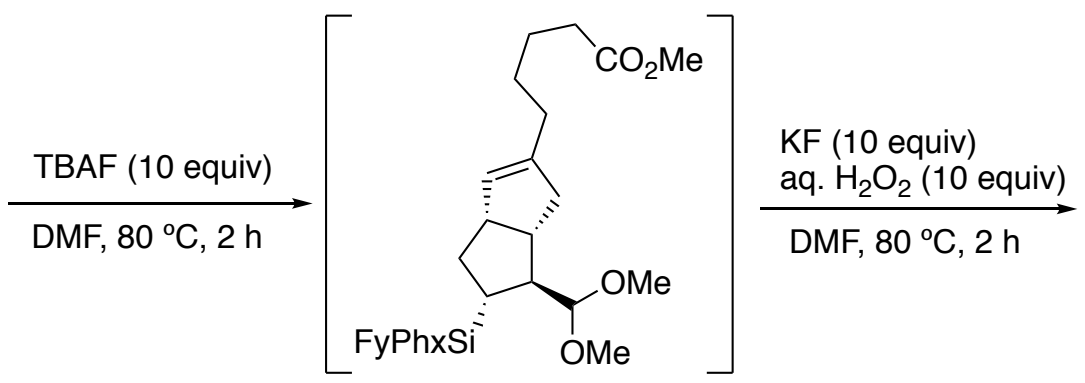

$(x, y)=(0,3),(1,2),(2,1)$
KF (10 equiv)

a. $\mathrm{H}_{2} \mathrm{O}_{2}$ (10 equiv)

$\mathrm{DMF}, 80^{\circ} \mathrm{C}, 2 \mathrm{~h}$<smiles>COC(=O)CCCCC1=C[C@@H]2C[C@H](O)[C@H](C(OC)OC)[C@@H]2C1</smiles>

23

\section{Mel (3.0 eq.) \\ DMF, $80{ }^{\circ} \mathrm{C}, 10 \mathrm{~min}$}

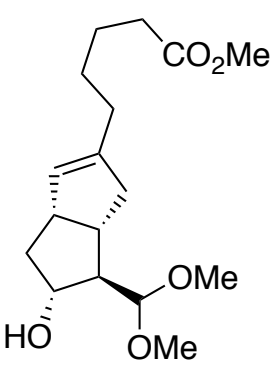

24

To a solution of compound $21(27.7 \mathrm{mg}, 0.050 \mathrm{mmol})$ in DMF $(100 \mu \mathrm{L})$, TBAF $(500 \mu \mathrm{L}, 1.0 \mathrm{M}$ in DMF, $0.50 \mathrm{mmol}$ ) was added at $80{ }^{\circ} \mathrm{C}$, which was heated by an oil bath. After stirring the reaction mixture at this temperature for $2 \mathrm{~h}$, to a reaction mixture, $\mathrm{KF}(28 \mathrm{mg}, 0.5 \mathrm{mmol})$ and aq. $\mathrm{H}_{2} \mathrm{O}_{2}(17.5 \mu \mathrm{L}, 0.50 \mathrm{mmol}, 35 \mathrm{wt}$ \% in $\mathrm{H}_{2} \mathrm{O}$ ) were added at $80{ }^{\circ} \mathrm{C}$, which was heated by an oil bath. After stirring the reaction mixture at this temperature for $2 \mathrm{~h}$, to a reaction mixture, $\mathrm{MeI}(9.3 \mu \mathrm{L}, 0.15 \mathrm{mmol})$ was added at $80{ }^{\circ} \mathrm{C}$, which was heated by an oil bath. After stirring the reaction mixture at this temperature for $10 \mathrm{~min}$, the reaction mixture was quenched by the addition of aq. sat. sodium thiosulfate $(1.0 \mathrm{~mL})$ and aq. sat. $\mathrm{NH}_{4} \mathrm{Cl}(1.0 \mathrm{~mL})$ at $0{ }^{\circ} \mathrm{C}$ and extracted with EtOAc $(3 \times 5 \mathrm{~mL})$. The combined organic extracts were washed with sat. aq. $\mathrm{NaCl}$ solution $(10$ $\mathrm{mL}$ ). Then, the combined organic extracts were dried with anhydrous $\mathrm{Na}_{2} \mathrm{SO}_{4}$, filtered, and concentrated under reduced pressure. After concentration in vacuo, the reaction mixture was purified by column chromatography on silica gel $(n$-Hexane: EtOAc $=4: 1)$ to give the target compound $\mathbf{2 4}$ in $81 \%$ yield $(12.6 \mathrm{mg}, 0.041 \mathrm{mmol})$. 
yl)pentanoate (24)

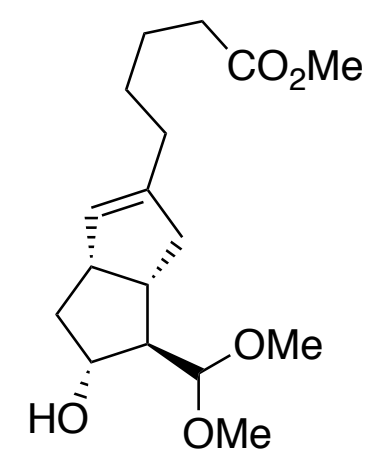

Yield: $81 \%(12.6 \mathrm{mg})$

Physical State: Colorless oil

${ }^{1} \mathbf{H}$ NMR $\left(\mathbf{C D C l}_{3}, 400 \mathrm{MHz}\right) \delta 5.28(\mathrm{~d}, 1 \mathrm{H}, J=1.6 \mathrm{~Hz}), 4.30$ (d, 1H, $\left.J=2.8 \mathrm{~Hz}\right), 3.89$ (ddd, 1H, $J=6.8,8.8$, $9.6 \mathrm{~Hz}), 3.65(\mathrm{~s}, 3 \mathrm{H}), 3.41(\mathrm{~s}, 3 \mathrm{H}), 3.37$ (s, 3H), $2.96(\mathrm{q}, 2 \mathrm{H}, J=8.4,15.6 \mathrm{~Hz}), 2.51(\mathrm{dd}, 1 \mathrm{H}, J=9.2,16.4 \mathrm{~Hz})$, $2.37(\mathrm{ddd}, 1 \mathrm{H}, J=2.8,9.2,18.4 \mathrm{~Hz}), 2.30(\mathrm{t}, 2 \mathrm{H}, J=7.6 \mathrm{~Hz}), 2.23(\mathrm{ddd}, 1 \mathrm{H}, J=6.8,8.0,12.0 \mathrm{~Hz}), 2.15(\mathrm{dd}$, $1 \mathrm{H}, J=0.80,16.4 \mathrm{~Hz}), 2.04(\mathrm{t}, 2 \mathrm{H}, J=7.6 \mathrm{~Hz}), 1.81(\mathrm{q}, 1 \mathrm{H}, J=8.8 \mathrm{~Hz}), 1.63($ quin, $2 \mathrm{H}, J=8.0 \mathrm{~Hz}), 1.44$ (quin, $2 \mathrm{H}, J=7.6 \mathrm{~Hz}), 1.31(\mathrm{ddd}, 1 \mathrm{H}, J=8.4,9.6,12.0 \mathrm{~Hz})$

${ }^{13} \mathbf{C} \mathbf{N M R}\left(\mathbf{C D C l}_{3}, \mathbf{1 0 0} \mathrm{MHz}\right) \delta 174.1,141.6,127.7,109.1,74.7,55.6,54.8,53.5,51.4,46.3,41.4,41.2,39.6$, $33.9,30.5,27.1,24.6$

HRMS (ESI): [M+Na] $]^{+}$calcd for $\mathrm{C}_{17} \mathrm{H}_{28} \mathrm{O}_{5} \mathrm{Na}: 335.1829$, found: 335.1825

IR(neat) $v 2920,1736,1457,1260,1064,800 \mathrm{~cm}^{-1}$

$[\alpha]_{\mathrm{D}}{ }^{26}-0.80\left(c 0.40, \mathrm{CHCl}_{3}\right)$

$\mathbf{R}_{\mathbf{f}}(n$-Hexane: EtOAc $=2: 1$, color reagent: Hanessian's stain reagent $): 0.30$

\subsection{Deprotection \& $\mathrm{HWE}$ reaction of compound 24}

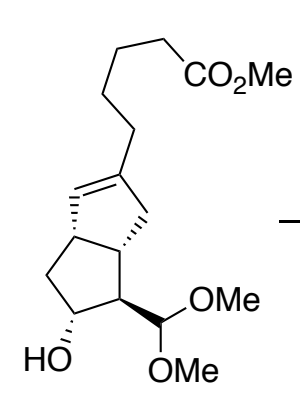

24

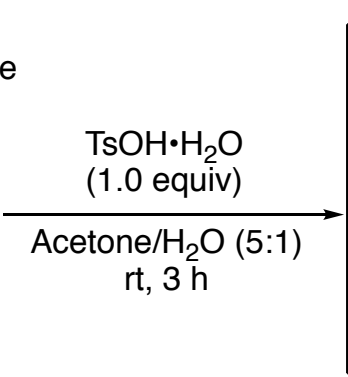

HO

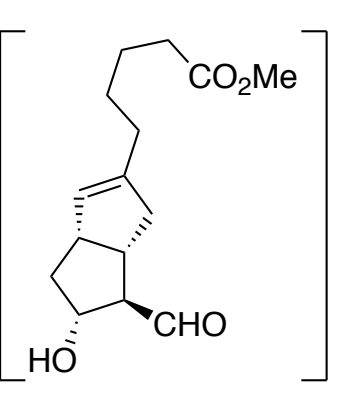

25

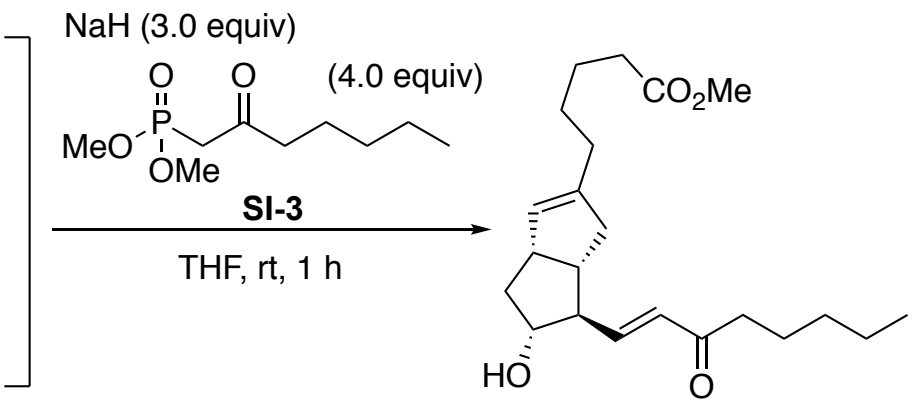

26

To a solution of compound $24(6.0 \mathrm{mg}, 0.019 \mathrm{mmol})$ in actetone $/ \mathrm{H}_{2} \mathrm{O}(5: 1,500 \mu \mathrm{L}), \mathrm{TsOH} \cdot \mathrm{H}_{2} \mathrm{O}(3.7 \mathrm{mg}$, 0.019) was added at room temperature. After stirring the reaction mixture at this temperature for $3 \mathrm{~h}$, to a reaction mixtute, a solution of compound $\mathbf{S I - 3 ^ { [ 3 ] }}(16.8 \mathrm{mg}, 0.076 \mathrm{mmol})$ and $\mathrm{NaH}(1.36 \mathrm{mg}, 0.057 \mathrm{mmol})$ in THF (1.0 mL) was added at room temperature. After stirring the reaction mixture at this temperature for $1 \mathrm{~h}$, the reaction mixture was quenched by the addition of aq. sat. $\mathrm{NH}_{4} \mathrm{Cl}(1.0 \mathrm{~mL})$ at $0{ }^{\circ} \mathrm{C}$ and extracted with 
EtOAc $(3 \times 5 \mathrm{~mL})$. The combined organic extracts were washed with sat. aq. $\mathrm{NaCl}$ solution $(10 \mathrm{~mL})$. Then, the combined organic extracts were dried with anhydrous $\mathrm{Na}_{2} \mathrm{SO}_{4}$, filtered, and concentrated under reduced pressure. After concentration in vacuo, the reaction mixture was purified by column chromatography on silica gel ( $n$-Hexane: EtOAc $=4: 1)$ to give the target compound 26 in $81 \%$ yield $(5.57 \mathrm{mg}, 0.015 \mathrm{mmol})$.

Methyl 5-((3aS,5R,6R)-5-hydroxy-6-((E)-3-oxooct-1-en-1-yl)-1,3a,4,5,6,6a-hexahydropentalen-2yl)pentanoate (26)

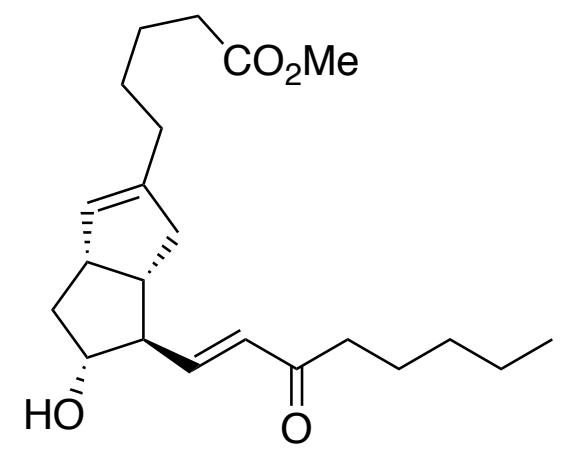

Yield: $81 \%(5.57 \mathrm{mg})$

Physical State: Colorless oil

${ }^{1} \mathbf{H}$ NMR $\left(\mathbf{C D C l}_{3}, 400 \mathrm{MHz}\right) \delta 6.76(\mathrm{~d}, 1 \mathrm{H}, J=8.8,15.6 \mathrm{~Hz}), 6.20(\mathrm{dd}, 1 \mathrm{H}, J=0.80,15.6 \mathrm{~Hz}), 5.31(\mathrm{~d}, 1 \mathrm{H}$, $J=1.6 \mathrm{~Hz}), 3.93(\mathrm{q}, 1 \mathrm{H}, J=9.2 \mathrm{~Hz}), 3.67(\mathrm{~s}, 3 \mathrm{H}), 3.07(\mathrm{~d}, 1 \mathrm{H}, J=6.8 \mathrm{~Hz}), 2.55(\mathrm{t}, 2 \mathrm{H}, J=7.6 \mathrm{~Hz}), 2.43-2.47$ $(\mathrm{m}, 2 \mathrm{H}), 2.31(\mathrm{t}, 2 \mathrm{H}, J=7.6 \mathrm{~Hz}), 2.29-2.39(\mathrm{~m}, 1 \mathrm{H}), 2.16(\mathrm{q}, 2 \mathrm{H}, J=8.4 \mathrm{~Hz}), 2.04(\mathrm{q}, 2 \mathrm{H}, J=7.6 \mathrm{~Hz}), 1.62$ (quin, $5 \mathrm{H}, J=7.6 \mathrm{~Hz}), 1.47(\mathrm{q}, 2 \mathrm{H}, J=7.2 \mathrm{~Hz}), 1.25-1.39(\mathrm{~m}, 5 \mathrm{H}), 0.878-0.912(\mathrm{~m}, 3 \mathrm{H})$

${ }^{13} \mathrm{C}$ NMR (CDCl ${ }_{3}, 100$ MHz) $\delta$ 200.5, 174.1, 147.3, 141.5, 131.1, 128.1, 77.2, 58.1, 51.5, 46.0, 44.3, 40.5, $40.2,39.8,33.9,31.4,30.5,27.1,24.6,23.8,22.5,13.9$

HRMS (ESI): $[\mathrm{M}+\mathrm{Na}]^{+}$calcd for $\mathrm{C}_{22} \mathrm{H}_{34} \mathrm{O}_{4} \mathrm{Na}$ : 385.2349 , found: 385.2354

$\operatorname{IR}($ neat $) v 2928,1739,1669,1437,1175,1092,476,447,435,411 \mathrm{~cm}^{-1}$

$[\alpha]_{\mathrm{D}}{ }^{26}+7.75\left(c 0.55, \mathrm{CHCl}_{3}\right)$

$\mathbf{R}_{\mathbf{f}}(n$-Hexane: EtOAc $=2: 1$, color reagent: Hanessian's stain reagent $): 0.50$

\subsection{Stereoselective reduction of compound 26}

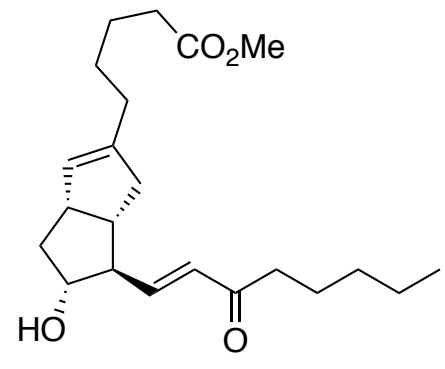

26

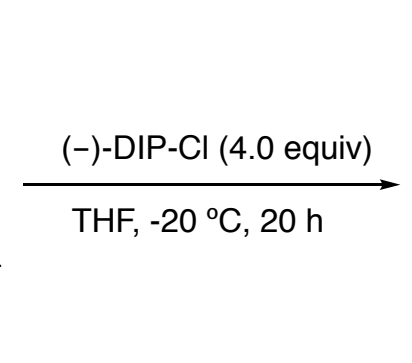

$\mathrm{HO}$

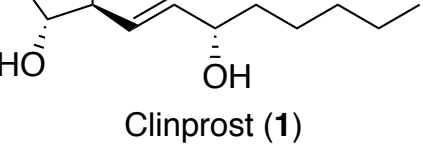

To a solution of compound 26 (5.0 mg, $0.013 \mathrm{mmol})$ in THF (1.0 mL), (-)-DIPCl (53 $\mu \mathrm{L}, 0.053 \mathrm{mmol}, 1.7$ $\mathrm{M}$ in $n$-Hexane) was added at $-20^{\circ} \mathrm{C}$. After stirring the reaction mixture at this temperature for $1 \mathrm{~h}$, the reaction 
mixture was quenched by the addition of aq. sat. $\mathrm{NH}_{4} \mathrm{Cl}(1.0 \mathrm{~mL})$ at $-20{ }^{\circ} \mathrm{C}$ and extracted with EtOAc $(3 \times 5$ $\mathrm{mL})$. The combined organic extracts were washed with sat. aq. $\mathrm{NaCl}$ solution $(10 \mathrm{~mL})$. Then, the combined organic extracts were dried with anhydrous $\mathrm{Na}_{2} \mathrm{SO}_{4}$, filtered, and concentrated under reduced pressure. After concentration in vacuo, the reaction mixture was purified by column chromatography on silica gel ( $n$-Hexane: EtOAc $=1: 1)$ to give the target compound 1 in $85 \%$ yield $(4.0 \mathrm{mg}, 0.011 \mathrm{mmol})$. All spectrum date was matched to reported one. ${ }^{[4](a)}$

Methyl 5-((3aS,5R,6R)-5-hydroxy-6-((S,E)-3-hydroxyoct-1-en-1-yl)-1,3a,4,5,6,6a-hexahydropentalen-2yl)pentanoate (1)

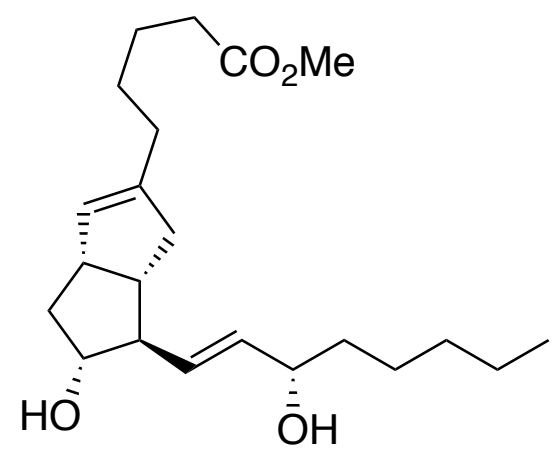

Yield: $85 \%(4.0 \mathrm{mg})$

Physical State: Colorless oil

${ }^{1} \mathbf{H}$ NMR $\left(\mathbf{C D C l}_{3}, 400 \mathrm{MHz}\right) \delta$ 5.53-5.64 (m, 2H), $5.29(\mathrm{~d}, 1 \mathrm{H}, J=1.6 \mathrm{~Hz}), 4.11$ (quin, $\left.1 \mathrm{H}, J=7.2 \mathrm{~Hz}\right), 3.77$ $(\mathrm{q}, 1 \mathrm{H}, J=9.2 \mathrm{~Hz}), 3.67(\mathrm{~s}, 3 \mathrm{H}), 3.01(\mathrm{q}, 1 \mathrm{H}, J=9.2 \mathrm{~Hz}), 2.43(\mathrm{~m}, 1 \mathrm{H}, J=8.8 \mathrm{~Hz}), 2.32(\mathrm{t}, 2 \mathrm{H}, J=7.6 \mathrm{~Hz})$, 2.27-2.37 (m, 2H), 2.00-2.06 (m, 3H), $1.94(\mathrm{dt}, 1 \mathrm{H}, J=7.2,9.2 \mathrm{~Hz}), 1.41-1.66(\mathrm{~m}, 6 \mathrm{H}), 1.24-1.34(\mathrm{~m}, 9 \mathrm{H})$, 0.882-0.905 (m, 3H)

${ }^{13} \mathrm{C}$ NMR $\left(\mathbf{C D C l}_{3}, \mathbf{1 0 0} \mathbf{M H z}\right) \delta 174.2,141.5,135.4,132.2,128.3,77.5,72.8,58.1,51.5,45.7,44.3,39.8$, $39.7,37.4,33.9,31.6,30.5,27.2,25.2,24.7,22.6,14.1$

HRMS (ESI): [M+Na] $]^{+}$calcd for $\mathrm{C}_{22} \mathrm{H}_{36} \mathrm{O}_{4} \mathrm{Na}$ : 387.2505 , found: 387.2505

IR(neat) $v$ 3385, 2927, 2859, 1740, 1438, 1258, 1201, 1173, 1089, $912 \mathrm{~cm}^{-1}$

$[\alpha]_{\mathrm{D}}{ }^{26}+9.50(c 0.40, \mathrm{MeOH})$, literature ${ }^{[4](b)} ;[\alpha]_{\mathrm{D}}{ }^{20}+10(c 0.55, \mathrm{MeOH})$

$\mathbf{R}_{\mathbf{f}}(n$-Hexane: EtOAc $=2: 1$, color reagent: Hanessian's stain reagent $): 0.30$ 


\subsection{Failed synthetic route of clinprost (Part 1)}

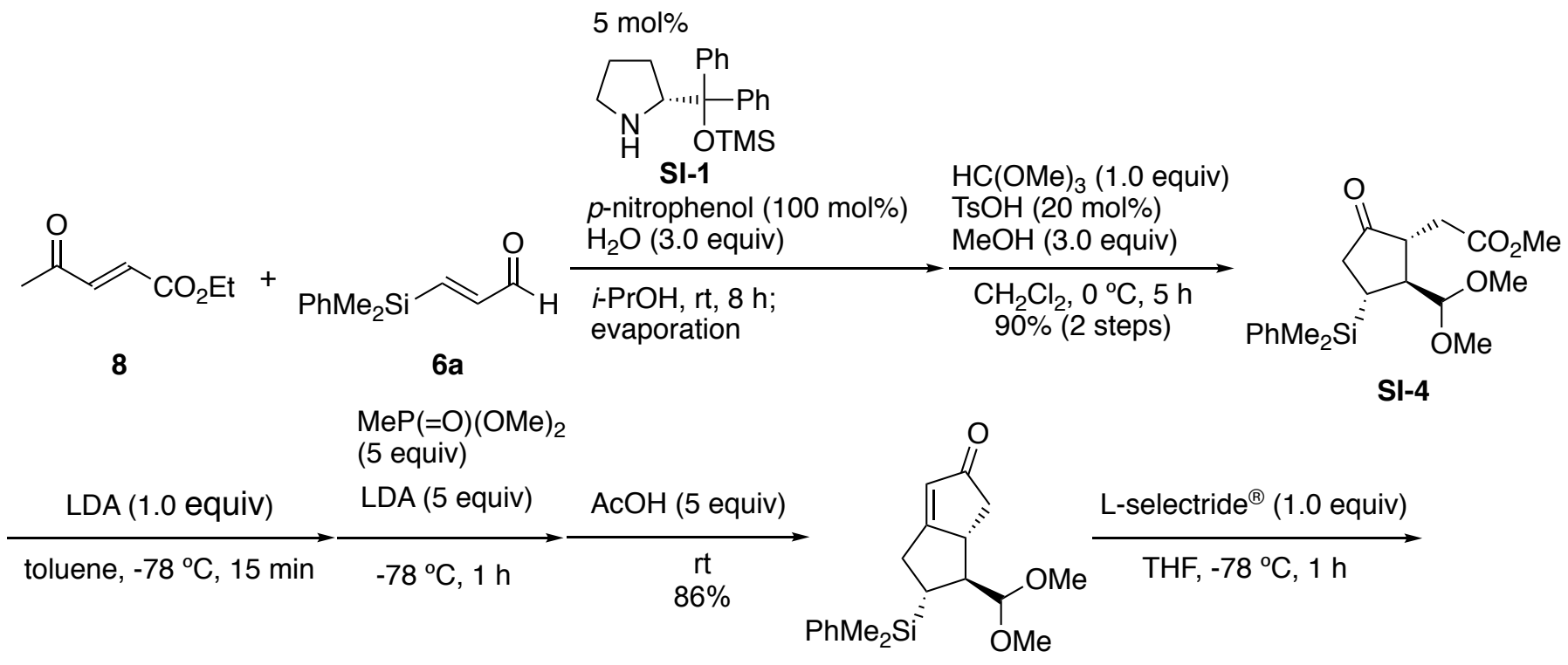

SI-5

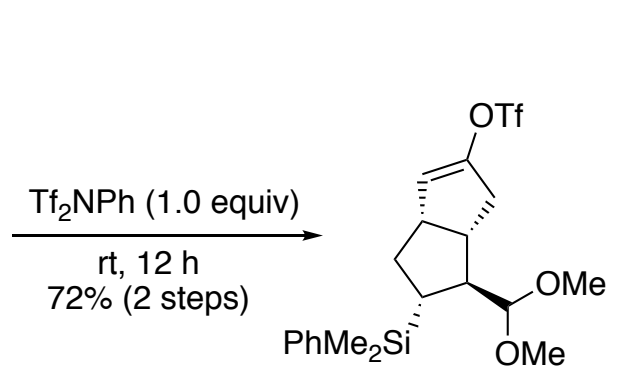

SI-6

$\mathrm{NaH}$ (3.0 equiv)
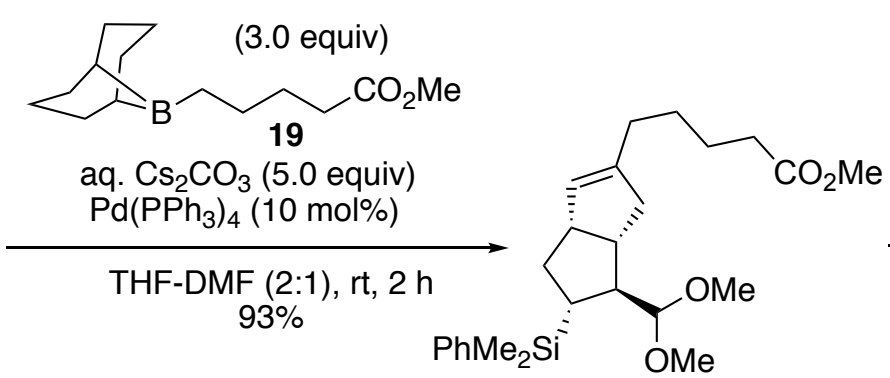

2N HCl/THF (1:3)

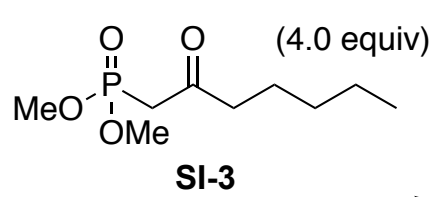

THF, rt, $8 \mathrm{~h}$

$86 \%$ (2 steps)
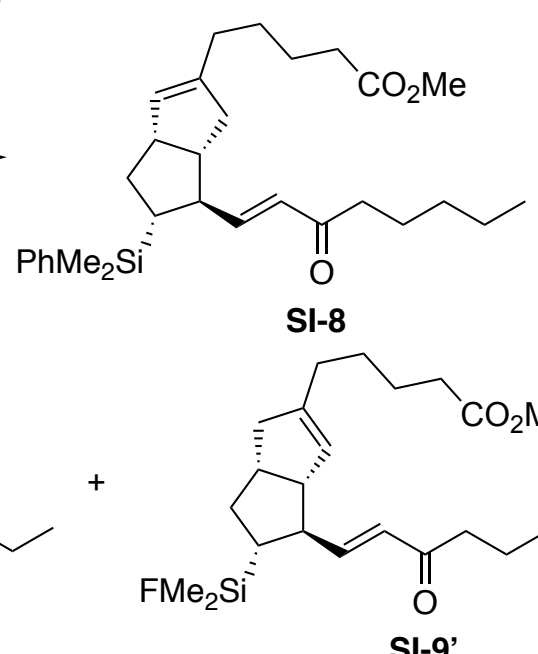

$\underset{\mathrm{CH}_{2} \mathrm{Cl}_{2}, \mathrm{rt}, 2 \mathrm{~h}}{\stackrel{\mathrm{BF}_{3} \cdot 2 \mathrm{AcOH}(10 \text { equiv })}{\longrightarrow}}$

SI-8
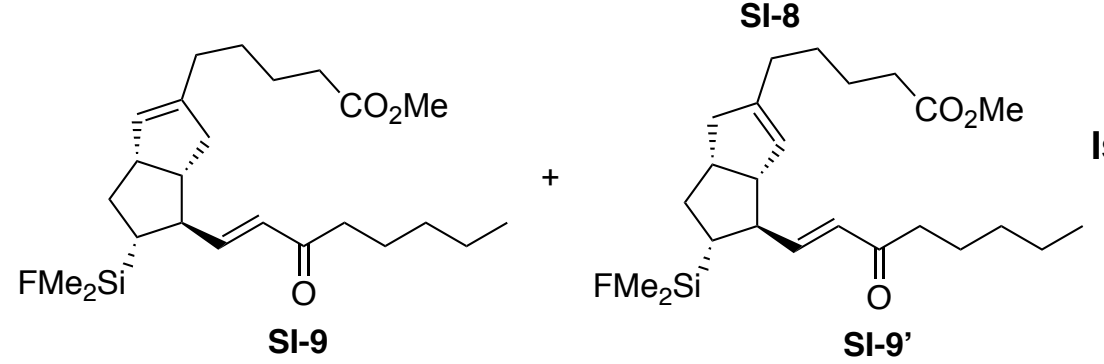

Isomerization of double-bond was observed in crude-NMR. 


\subsection{Asymmetric domino Michael/Michael reaction between ketone 8 and aldehyde $6 a$}

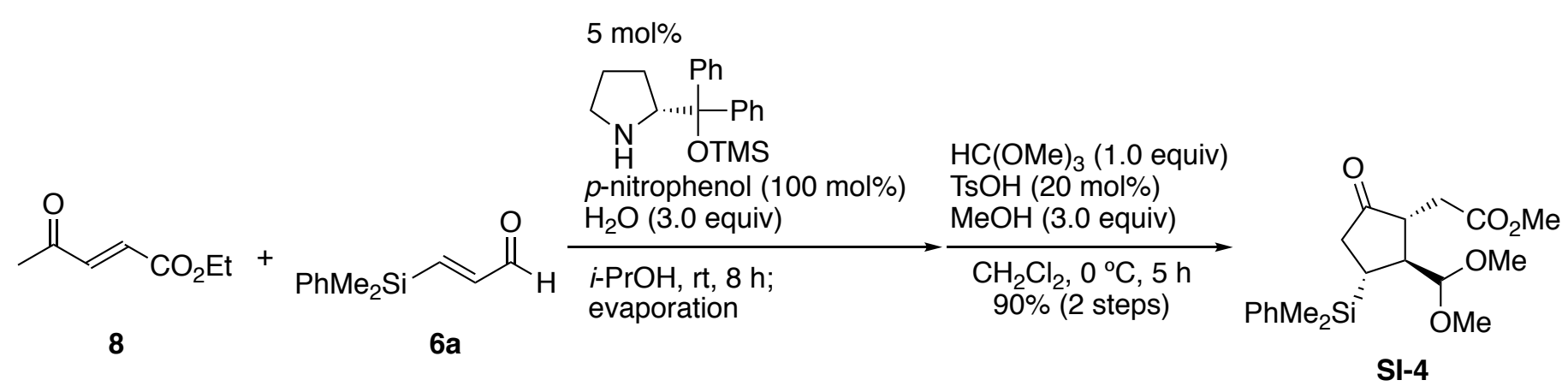

To a solution of ketone $\mathbf{8}^{[2]}(284 \mathrm{mg}, 2.0 \mathrm{mmol})$, aldehyde $\mathbf{6 a}^{[2]}(190 \mathrm{mg}, 1.0 \mathrm{mmol}), \mathrm{H}_{2} \mathrm{O}(540 \mu \mathrm{L}, 3.0$ mmol) and $p$-nitrophenol (139 mg, $1.0 \mathrm{mmol})$ in $i$-PrOH $(250 \mu \mathrm{L})$, organocatalyst SI-1 (16.5 mg, $0.050 \mathrm{mmol})$ was added at room temperature. After stirring the reaction mixture at this temperature for $8 \mathrm{~h}$, concentrated under reduced pressure. To a solution of residue in $\mathrm{CH}_{2} \mathrm{Cl}_{2}(2.5 \mathrm{~mL}), \mathrm{HC}(\mathrm{OMe})_{3}(109 \mu \mathrm{L}, 1.0 \mathrm{mmol}), \mathrm{MeOH}$ $(41 \mu \mathrm{L}, 1.0 \mathrm{mmol})$ and $\mathrm{TsOH} \cdot \mathrm{H}_{2} \mathrm{O}(36 \mathrm{mg}, 0.20 \mathrm{mmol})$ were added at $0{ }^{\circ} \mathrm{C}$. After stirring the reaction mixture at this temperature for $5 \mathrm{~h}$, the reaction mixture was quenched by the addition of sat. aq. $\mathrm{NaHCO}_{3}(5 \mathrm{~mL})$. Upon completion, $\mathrm{H}_{2} \mathrm{O}(2 \mathrm{~mL})$ was added and the mixture was extracted with EtOAc $(3 \times 5 \mathrm{~mL})$. The combined organic extracts were washed with sat. aq. $\mathrm{NaHCO}_{3}(5 \mathrm{~mL})$ and sat. aq. $\mathrm{NaCl}$ solution $(5 \mathrm{~mL})$. Then, the combined organic extracts were dried with anhydrous $\mathrm{Na}_{2} \mathrm{SO}_{4}$, filtered, and concentrated under reduced pressure. After concentration in vacuo, the reaction mixture was purified by column chromatography on silica gel $(n$-Hexane: EtOAc $=6: 1)$ to give the target compound SI-4 in 90\% yield (340 mg, $0.90 \mathrm{mmol})$.

\section{Methyl 2-((1R,2S,3R)-2-(dimethoxymethyl)-3-(dimethyl(phenyl)silyl)-5-oxocyclopentyl)acetate (SI-4)}<smiles>COC(OC)[C@H]1[C@@H](CC(C)=O)CC(=O)[C@H]1[SiH2]O</smiles>

Yield: $90 \%(340 \mathrm{mg})$

Physical State: Yellow oil

${ }^{1}$ H NMR (CDCl ${ }_{3}, 400$ MHz) $\delta 7.53$ (brs, 2H), 7.37 (brs, 3H), 3.91 (d, 1H, J = 3.6 Hz), 3.59 (s, 3H), 3.15 (s, $6 \mathrm{H}), 2.65-2.78(\mathrm{~m}, 2 \mathrm{H}), 2.56-2.59(\mathrm{~m}, 1 \mathrm{H}), 2.35-2.44(\mathrm{~m}, 2 \mathrm{H}), 2.18(\mathrm{dd}, 1 \mathrm{H}, J=13.2,18.8 \mathrm{~Hz}), 1.52(\mathrm{q}, 1 \mathrm{H}$, $J=12.4 \mathrm{~Hz}), 0.388(\mathrm{~s}, 3 \mathrm{H}), 0.330(\mathrm{~s}, 3 \mathrm{H})$

${ }^{13} \mathbf{C} \mathbf{N M R}\left(\mathbf{C D C l}_{3}, \mathbf{1 0 0} \mathrm{MHz}\right) \delta$ 219.0, 172.6, 138.0, 133.7, 129.3, 129.3, 127.9, 127.9, 107.3, 56.3, 56.1, 51.4, $46.5,45.6,40.0,33.7,21.7,-3.47,-5.26$

HRMS (ESI): $[\mathrm{M}+\mathrm{Na}]^{+}$calcd for $\mathrm{C}_{19} \mathrm{H}_{28} \mathrm{O}_{5} \mathrm{SiNa}: 387.1598$, found: 387.1598

IR(neat) $v 1739,1253,1197,1117,1075,839,738,702 \mathrm{~cm}^{-1}$

$[\alpha]_{\mathrm{D}}{ }^{26}-99.6\left(c 0.50, \mathrm{CHCl}_{3}\right)$

$\mathbf{R}_{\mathbf{f}}(n$-Hexane: EtOAc $=2: 1$, color reagent: Hanessian's stain reagent $): 0.60$ 


\subsection{One-pot procedure to synthesize compound SI-5}

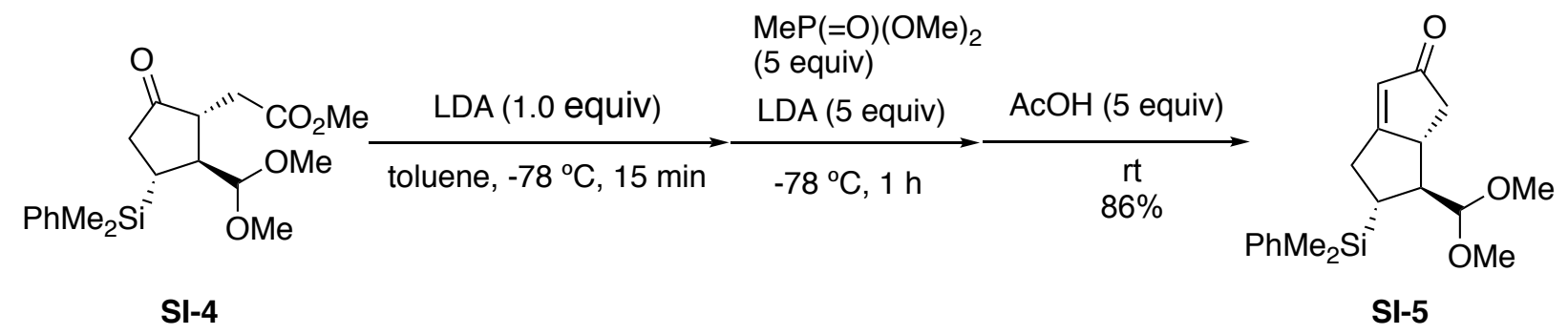

To a solution of compound SI-4 $(75.6 \mathrm{mg}, 0.20 \mathrm{mmol})$ in toluene $(2.0 \mathrm{~mL})$, LDA (200 $\mu \mathrm{L}, 1.0 \mathrm{M}$ in THF) was added at $-78{ }^{\circ} \mathrm{C}$. After stirring the reaction mixture at this temperature for $15 \mathrm{~min}$, to a reaction mixture, dimethyl methylphosphonate $(124 \mathrm{mg}, 1.0 \mathrm{mmol})$ and LDA $\left(1.0 \mathrm{~mL}, 1.0 \mathrm{M}\right.$ in THF) were added at $-78^{\circ} \mathrm{C}$. After stirring the reaction mixture at this temperature for $1 \mathrm{~h}$, to a reaction mixture, actetic acid (60 $\mathrm{mg}, 1.0$ $\mathrm{mmol})$ in toluene $(3.0 \mathrm{~mL})$ was added at $-78^{\circ} \mathrm{C}$. After stirring the reaction mixture at room temperature for 24 $\mathrm{h}, \mathrm{H}_{2} \mathrm{O}(5 \mathrm{~mL})$ was added and the mixture was extracted with EtOAc $(3 \times 10 \mathrm{~mL})$. The combined organic extracts were washed with sat. aq. $\mathrm{NaCl}$ solution $(40 \mathrm{~mL})$. Then, the combined organic extracts were dried with anhydrous $\mathrm{Na}_{2} \mathrm{SO}_{4}$, filtered, and concentrated under reduced pressure. After concentration in vacuo, the reaction mixture was purified by column chromatography on silica gel $(n$-Hexane: EtOAc $=3: 1)$ to give the target compound SI-5 in $86 \%$ yield (57.0 mg, $0.17 \mathrm{mmol})$.

\section{(5R,6S,6aR)-6-(Dimethoxymethyl)-5-(dimethyl(phenyl)silyl)-4,5,6,6a-tetrahydropentalen-2(1H)-one} (SI-5)

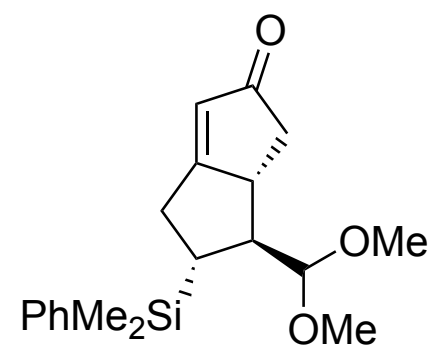

Yield: $86 \%(57.0 \mathrm{mg})$

Physical State: Yellow oil

${ }^{1} \mathbf{H}$ NMR (CDCl ${ }_{3}, 400$ MHz) 87.49-7.51 (m, 2H), 7.34-7.39 (m, 3H), 5.77 (dt, 1H, J = 1.2, $\left.2.0 \mathrm{~Hz}\right), 3.90$ (d, $1 \mathrm{H}, J=3.6 \mathrm{~Hz}), 3.27(\mathrm{~s}, 3 \mathrm{H}), 3.21(\mathrm{~s}, 3 \mathrm{H}), 3.03-3.09(\mathrm{~m}, 1 \mathrm{H}), 2.81(\mathrm{dd}, 1 \mathrm{H}, J=11.6,18.8 \mathrm{~Hz}), 2.62(\mathrm{dd}, 1 \mathrm{H}$, $J=6.4,18.4 \mathrm{~Hz}), 2.48(\mathrm{dd}, 1 \mathrm{H}, J=5.6,18.0 \mathrm{~Hz}), 2.08(\mathrm{dd}, 1 \mathrm{H}, J=3.2,18.0 \mathrm{~Hz}), 1.76-1.86(\mathrm{~m}, 2 \mathrm{H}), 0.309$ $(\mathrm{s}, 3 \mathrm{H}), 0.300(\mathrm{~s}, 3 \mathrm{H})$

${ }^{13} \mathbf{C} \mathbf{N M R}\left(\mathbf{C D C l}_{3}, \mathbf{1 0 0} \mathbf{M H z}\right) \delta 211.4,190.1,137.8,137.8,133.8,129.3,127.9,124.8,124.8,107.5,56.1$, $56.0,49.4,47.6,43.1,29.2,26.9,-4.05,-4.78$

HRMS (ESI): $[\mathrm{M}+\mathrm{Na}]^{+}$calcd for $\mathrm{C}_{19} \mathrm{H}_{26} \mathrm{O}_{3} \mathrm{SiNa}$ : 353.1543, found: 353.1543

IR(neat) $v 1705,1628,1251,1182,1115,1075,814,775,737,702 \mathrm{~cm}^{-1}$

$[\alpha]_{\mathrm{D}}{ }^{26}-75.2\left(c 2.1, \mathrm{CHCl}_{3}\right)$

$\mathbf{R}_{\mathbf{f}}(n$-Hexane: EtOAc $=2: 1$, color reagent: Hanessian's stain reagent $): 0.50$ 


\subsection{One-pot synthesis of compound SI-6 from compound SI-5}

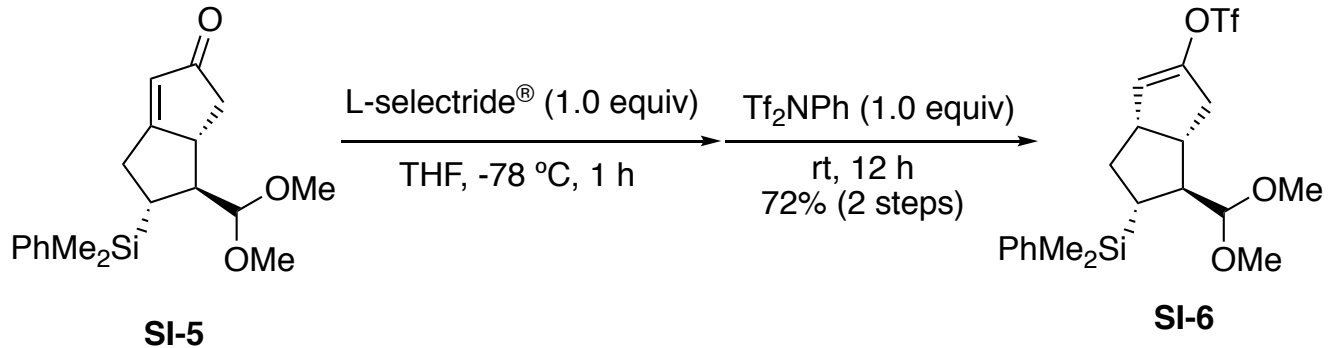

To a solution of compound SI-5 $(33.0 \mathrm{mg}, 0.10 \mathrm{mmol})$ in THF $(1.0 \mathrm{~mL})$, L-selectride ${ }^{\circledR}(120 \mu \mathrm{L}, 1.0 \mathrm{M}$ in THF) was added at $-78{ }^{\circ} \mathrm{C}$. After stirring the reaction mixture at this temperature for $1 \mathrm{~h}$, to a reaction mixture, $\mathrm{Tf}_{2} \mathrm{NPh}(43 \mathrm{mg}, 0.10 \mathrm{mmol})$ was added at $-78^{\circ} \mathrm{C}$. After stirring the reaction mixture at this temperature for 1 $\mathrm{h}$, the reaction was quenched by the addition of buffer $(1.0 \mathrm{~mL})$ and extracted with EtOAc $(3 \times 5 \mathrm{~mL})$. The combined organic extracts were washed with sat. aq. $\mathrm{NaCl}$ solution $(10 \mathrm{~mL})$. Then, the combined organic extracts were dried with anhydrous $\mathrm{Na}_{2} \mathrm{SO}_{4}$, filtered, and concentrated under reduced pressure. After concentration in vacuo, the reaction mixture was purified by column chromatography on silica gel ( $n$-Hexane: EtOAc $=20: 1)$ to give the target compound SI-6 in $72 \%$ yield $(33.4 \mathrm{mg}, 0.072 \mathrm{mmol})$.

\section{(3aS,5R,6S)-6-(Dimethoxymethyl)-5-(triphenylsilyl)-1,3a,4,5,6,6a-hexahydropentalen-2-yl}

\section{trifluoromethanesulfonate (SI-6)}<smiles>CCC[C@@H]1C=C([Al-])C[C@@H]1C(OC)OC</smiles>

Yield: $72 \%(33.4 \mathrm{mg})$

Physical State: Colorless oil

${ }^{1} \mathbf{H}$ NMR $\left(\mathbf{C D C l}_{\mathbf{3}}, \mathbf{4 0 0} \mathbf{M H z}\right) \delta$ 7.48-7.54 (m, 2H), 7.33-7.37 (m, 3H), $5.54(\mathrm{~d}, 1 \mathrm{H}, J=2.4 \mathrm{~Hz}), 3.94(\mathrm{~d}, 1 \mathrm{H}$, $J=4.0 \mathrm{~Hz}), 3.19$ (s, 3H), 3.14 (s, 3H), 3.01-3.08 (m, 1H), 2.72-2.81 (m, 2H), 2.31-2.39 (m, 1H), 2.06-2.13 $(\mathrm{m}, 1 \mathrm{H}), 1.81(\mathrm{sep}, 1 \mathrm{H}, J=3.6 \mathrm{~Hz}), 1.14-1.28(\mathrm{~m}, 2 \mathrm{H}), 0.318(\mathrm{~s}, 3 \mathrm{H}), 0.273(\mathrm{~s}, 3 \mathrm{H})$

${ }^{13} \mathbf{C}$ NMR $\left(\mathbf{C D C l}_{3}, \mathbf{1 0 0} \mathbf{M H z}\right) \delta$ 146.8, 138.9, 133.7, 132.1, 131.0, 130.0, 128.9, 127.8, 121.2, 107.6, 56.0, $55.8,55.3,46.1,41.2,37.6,36.0,28.4,-3.34,-4.95$

HRMS (ESI): [M+Na] $]^{+}$calcd for $\mathrm{C}_{20} \mathrm{H}_{27} \mathrm{~F}_{3} \mathrm{O}_{5} \mathrm{SSiNa}$ : 487.1193, found: 487.1193

IR(neat) $v 2954,1658,1422,1212,1142,907,736,701,608,521 \mathrm{~cm}^{-1}$

$[\alpha]_{\mathrm{D}}{ }^{26}-34.6\left(c 1.3, \mathrm{CHCl}_{3}\right)$

$\mathbf{R}_{\mathbf{f}}(n$-Hexane: EtOAc $=10: 1$, color reagent: Hanessian's stain reagent): 0.75 


\subsection{Suzuki-Miyaura reaction of compound SI-6}

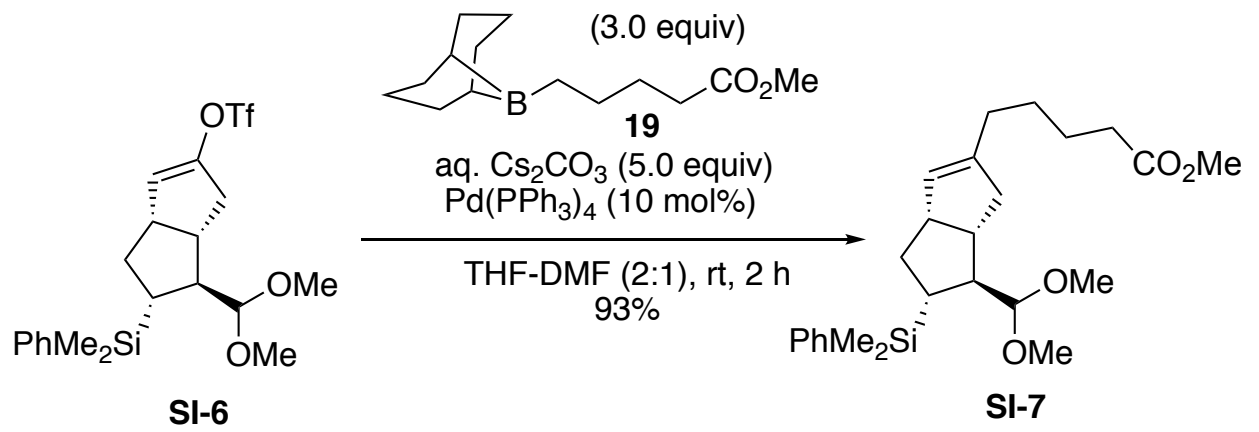

To a solution of methyl pent-4-enoate (52 mg, $0.46 \mathrm{mmol})$ in THF (2 mL), 9-BBN (3.0 mL, $0.5 \mathrm{M}$ in THF) was added at room temperature. After stirring the reaction mixture at this temperature for $1 \mathrm{~h}$, to a reaction mixture, aq. $\mathrm{Cs}_{2} \mathrm{CO}_{3}\left(91 \mu \mathrm{L}, 3.0 \mathrm{M}\right.$ in $\left.\mathrm{H}_{2} \mathrm{O}, 2.73 \mathrm{mmol}\right)$ was added. Then, to a reaction micture, compound SI-6 (214 mg, $0.46 \mathrm{mmol})$ in DMF (2 mL) and $\mathrm{Pd}\left(\mathrm{PPh}_{3}\right)_{4}(79 \mathrm{mg}, 0.068 \mathrm{mmol})$ in THF (2.0 mL) were added at room temperature. After stirring the reaction mixture at this temperature for $2 \mathrm{~h}$, the reaction was quenched by the addition of water $(5.0 \mathrm{~mL})$ and extracted with EtOAc $(3 \times 20 \mathrm{~mL})$. The combined organic extracts were washed with sat. aq. $\mathrm{NaCl}$ solution $(60 \mathrm{~mL})$. Then, the combined organic extracts were dried with anhydrous $\mathrm{Na}_{2} \mathrm{SO}_{4}$, filtered, and concentrated under reduced pressure. After concentration in vacuo, the reaction mixture was purified by column chromatography on silica gel $(n$-Hexane: EtOAc $=12: 1)$ to give the target compound SI-7 in $93 \%$ yield (180 mg, $0.42 \mathrm{mmol})$.

Methyl

5-((3aS,5R,6S,6aS)-6-(dimethoxymethyl)-5-(dimethyl(phenyl)silyl)-1,3a,4,5,6,6ahexahydropentalen-2-yl)pentanoate (SI-7)

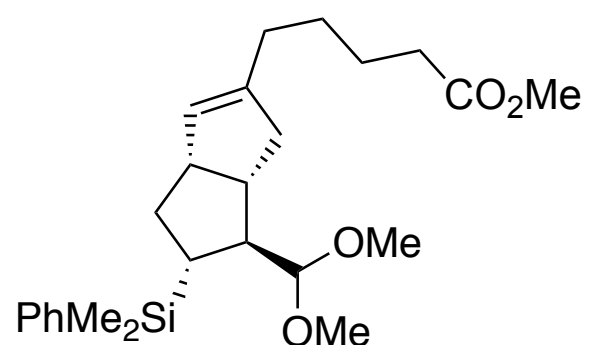

Yield: $93 \%$ (180 mg)

Physical State: Colorless oil

${ }^{1} \mathbf{H}$ NMR $\left(\mathbf{C D C l}_{3}, \mathbf{4 0 0} \mathbf{M H z}\right) \delta$ 7.48-7.51 (m, 2H), 7.31-7.33 (m, 3H), $5.18(\mathrm{~d}, 1 \mathrm{H}, J=1.6 \mathrm{~Hz}), 3.94(\mathrm{~d}, 1 \mathrm{H}$, $J=4.0 \mathrm{~Hz}), 3.66(\mathrm{~s}, 3 \mathrm{H}), 3.16(\mathrm{~s}, 3 \mathrm{H}), 3.16(\mathrm{~s}, 3 \mathrm{H}), 2.39-2.42(\mathrm{~m}, 2 \mathrm{H}), 2.30(\mathrm{t}, 2 \mathrm{H}, J=7.6 \mathrm{~Hz}), 1.95-2.02(\mathrm{~m}$, $2 \mathrm{H}), 1.84-1.90(\mathrm{~m}, 2 \mathrm{H}), 1.72-1.77(\mathrm{~m}, 1 \mathrm{H}), 1.51-1.61(\mathrm{~m}, 3 \mathrm{H}), 1.37-1.46(\mathrm{~m}, 2 \mathrm{H}), 1.25(\mathrm{ddd}, 1 \mathrm{H}, J=6.8,11.6$, $13.2 \mathrm{~Hz}), 1.10(\mathrm{dt}, 1 \mathrm{H}, J=8.0,12.8 \mathrm{~Hz}), 0.283(\mathrm{~s}, 3 \mathrm{H}), 0.231(\mathrm{~s}, 3 \mathrm{H})$,

${ }^{13} \mathbf{C ~ N M R}\left(\mathbf{C D C l}_{3}, \mathbf{1 0 0} \mathbf{M H z}\right) \delta$ 174.2, 141.6, 139.9, 133.7, 133.7, 128.5, 127.5, 127.5, 108.6, 55.6, 55.3, 53.2, $51.5,44.5,41.9,41.5,36.5,33.9,30.6,29.4,27.1,25.6,24.6,-3.25,-4.60$

HRMS (ESI): $[\mathrm{M}+\mathrm{Na}]^{+}$calcd for $\mathrm{C}_{25} \mathrm{H}_{38} \mathrm{O}_{4} \mathrm{SiNa}$ : 453.2432, found: 453.2437

IR(neat) $v 1740,1437,1083,817,735,701 \mathrm{~cm}^{-1}$

$[\alpha]_{\mathrm{D}}{ }^{26}-42.1\left(\right.$ c $\left.1.5, \mathrm{CHCl}_{3}\right)$ 


\subsection{Deprotection \& HWE reaction of compound SI-7}

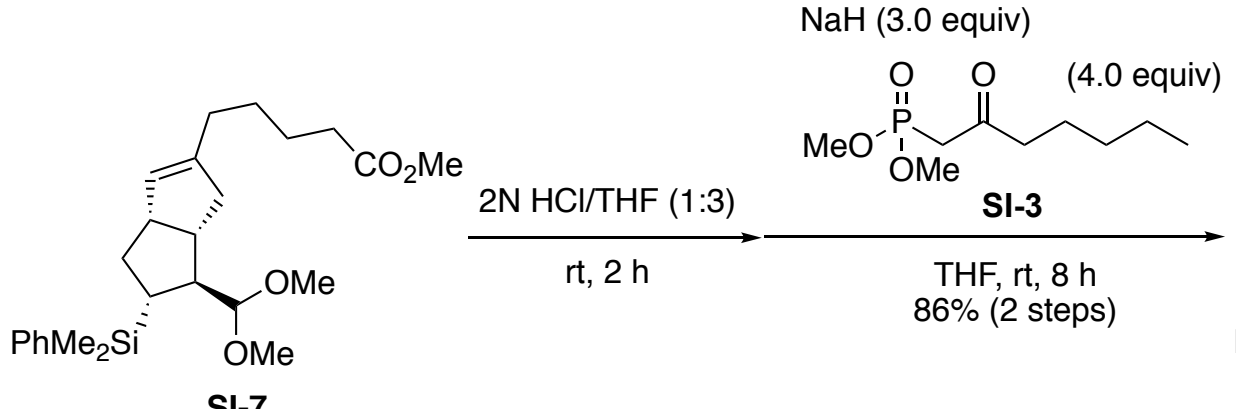

SI-7

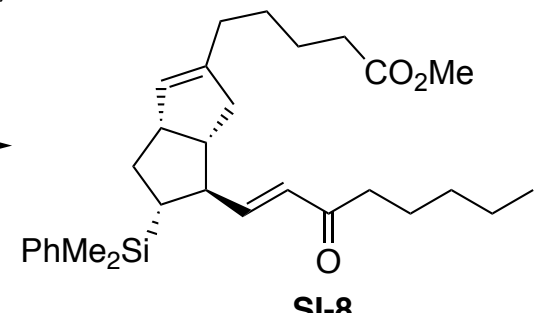

SI-8

To a solution of compound SI-7 $(86.0 \mathrm{mg}, 0.20 \mathrm{mmol})$ in actetone $/ \mathrm{H}_{2} \mathrm{O}(5: 1,5.0 \mathrm{~mL}), \mathrm{TsOH} \cdot \mathrm{H}_{2} \mathrm{O}(37 \mathrm{mg}$, 0.20) was added at room temperature. After stirring the reaction mixture at this temperature for $3 \mathrm{~h}$, to a reaction mixtute, a solution of compound $\mathbf{S I - 3}{ }^{[3]}(168 \mathrm{mg}, 0.76 \mathrm{mmol})$ and $\mathrm{NaH}(13.6 \mathrm{mg}, 0.57 \mathrm{mmol})$ in THF $(10 \mathrm{~mL})$ were added at room temperature. After stirring the reaction mixture at this temperature for $1 \mathrm{~h}$, the reaction mixture was quenched by the addition of aq. sat. $\mathrm{NH}_{4} \mathrm{Cl}(10 \mathrm{~mL})$ at $0{ }^{\circ} \mathrm{C}$ and extracted with EtOAc $(3 \times 10 \mathrm{~mL})$. The combined organic extracts were washed with sat. aq. $\mathrm{NaCl}$ solution $(30 \mathrm{~mL})$. Then, the combined organic extracts were dried with anhydrous $\mathrm{Na}_{2} \mathrm{SO}_{4}$, filtered, and concentrated under reduced pressure. After concentration in vacuo, the reaction mixture was purified by column chromatography on silica gel ( $n$-Hexane: EtOAc $=7: 1)$ to give the target compound $\mathbf{S I - 8}$ in $86 \%$ yield $(82.7 \mathrm{mg}, 0.17 \mathrm{mmol})$.

Methyl 5-((3aS,5R,6R,6aS)-5-(dimethyl(phenyl)silyl)-6-((E)-3-oxooct-1-en-1-yl)-1,3a,4,5,6,6ahexahydropentalen-2-yl)pentanoate (SI-8)

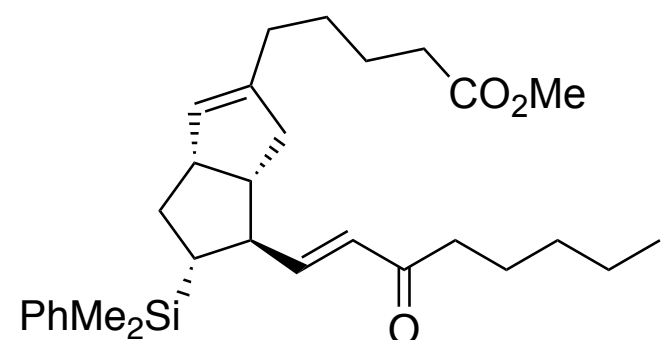

Yield: $86 \%(82.7 \mathrm{mg})$

Physical State: Colorless oil

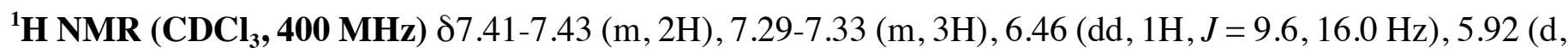
$1 \mathrm{H}, J=16.0 \mathrm{~Hz}), 5.78(\mathrm{~s}, 1 \mathrm{H}), 3.67(\mathrm{~s}, 3 \mathrm{H}), 3.17$ (brs, 1H), $2.30(\mathrm{t}, 2 \mathrm{H}, J=7.6 \mathrm{~Hz}), 2.08-2.12(\mathrm{~m}, 1 \mathrm{H}), 1.98$ $2.02(\mathrm{~m}, 2 \mathrm{H}), 1.82-1.90(\mathrm{~m}, 2 \mathrm{H}), 1.50-1.60(\mathrm{~m}, 5 \mathrm{H}), 1.42(\mathrm{q}, 2 \mathrm{H}, J=7.2 \mathrm{~Hz}), 1.22-1.31(\mathrm{~m}, 8 \mathrm{H}), 0.890(\mathrm{q}$, $4 \mathrm{H}, J=6.8 \mathrm{~Hz}), 0.227(\mathrm{~s}, 3 \mathrm{H}), 0.218(\mathrm{~s}, 3 \mathrm{H})$

${ }^{13} \mathbf{C} \mathbf{N M R}\left(\mathbf{C D C l}_{3}, \mathbf{1 0 0} \mathbf{M H z}\right) \delta 200.9,174.1,151.0,141.1,138.4,133.8,133.8,129.7,128.9,128.1,127.6$, $127.6,53.8,51.5,51.0,39.1,38.3,35.7,34.7,33.9,31.6,31.5,30.6,27.1,24.6,23.8,22.5,14.0,-3.92,-4.65$ HRMS (ESI): $[\mathrm{M}+\mathrm{Na}]^{+}$calcd for $\mathrm{C}_{30} \mathrm{H}_{44} \mathrm{O}_{3} \mathrm{SiNa}$ : 503.2952, found: 503.2952 $\operatorname{IR}\left(\right.$ neat) $v 1740,1673,1626,1428,1250,816,735,701 \mathrm{~cm}^{-1}$ 
$[\alpha]_{\mathrm{D}}{ }^{26}-40.4\left(c 1.1, \mathrm{CHCl}_{3}\right)$

$\mathbf{R}_{\mathbf{f}}(n$-Hexane: EtOAc $=2: 1$, color reagent: Hanessian's stain reagent $): 0.63$

\subsection{Failed synthetic route of clinprost (Part 2)}

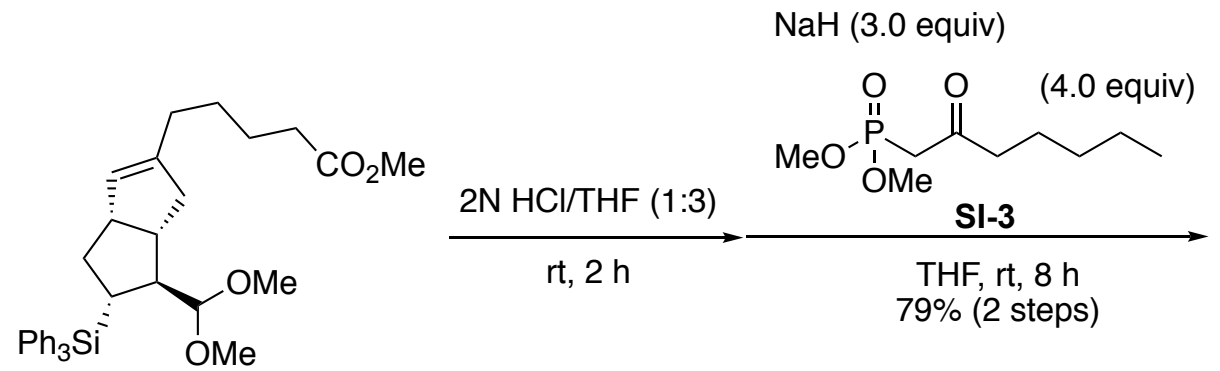

21

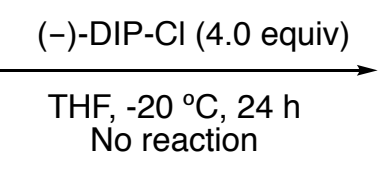

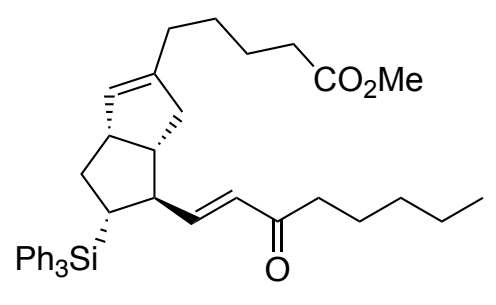

SI-10

\subsection{Deprotection \& $\mathrm{HWE}$ reaction of compound 21}

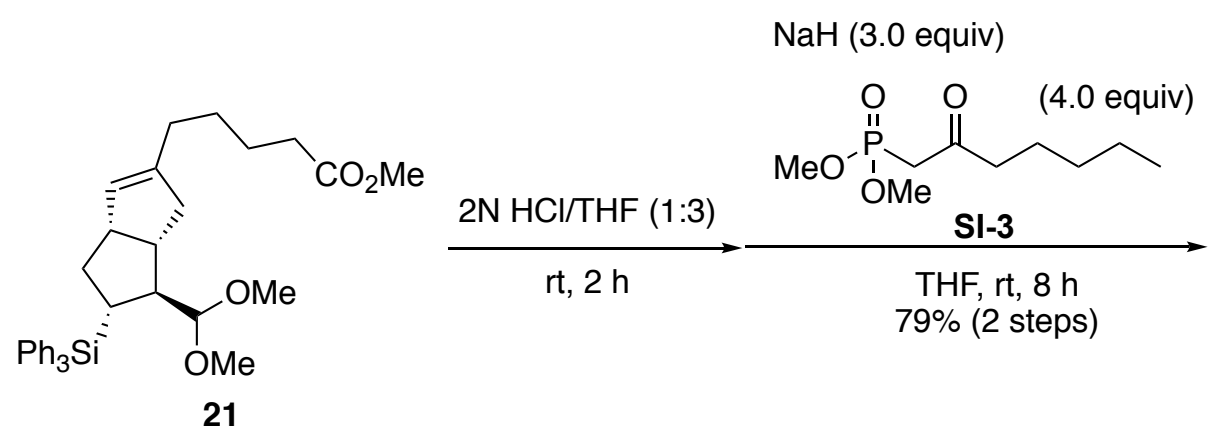

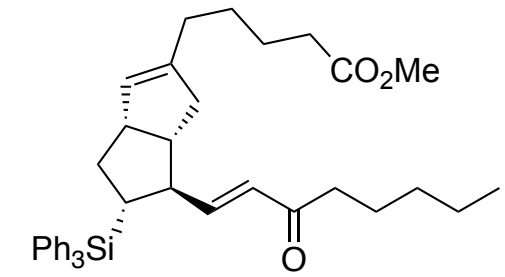

27

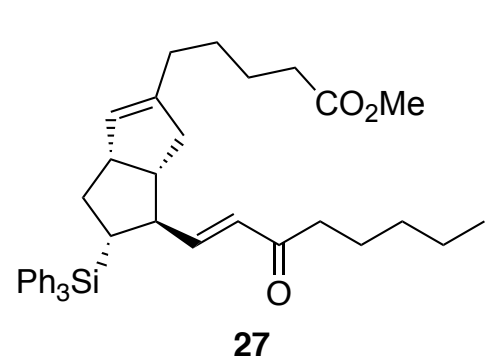

To a solution of compound $21(11.1 \mathrm{mg}, 0.020 \mathrm{mmol})$ in actetone $/ \mathrm{H}_{2} \mathrm{O}(5: 1,500 \mu \mathrm{L})$, TsOH$\cdot \mathrm{H}_{2} \mathrm{O}(3.8 \mathrm{mg}$, 0.020) was added at room temperature. After stirring the reaction mixture at this temperature for $3 \mathrm{~h}$, to a reaction mixtute, a solution of compound $\mathbf{S I - 3}{ }^{[3]}(17.6 \mathrm{mg}, 0.080 \mathrm{mmol})$ and $\mathrm{NaH}(1.40 \mathrm{mg}, 0.060 \mathrm{mmol})$ in THF $(1.0 \mathrm{~mL})$ was added at room temperature. After stirring the reaction mixture at this temperature for $1 \mathrm{~h}$, the reaction mixture was quenched by the addition of aq. sat. $\mathrm{NH}_{4} \mathrm{Cl}(1.0 \mathrm{~mL})$ at $0{ }^{\circ} \mathrm{C}$ and extracted with EtOAc $(3 \times 5 \mathrm{~mL})$. The combined organic extracts were washed with sat. aq. $\mathrm{NaCl}$ solution $(10 \mathrm{~mL})$. Then, the combined organic extracts were dried with anhydrous $\mathrm{Na}_{2} \mathrm{SO}_{4}$, filtered, and concentrated under reduced pressure. After concentration in vacuo, the reaction mixture was purified by column chromatography on silica gel $(n$-Hexane: EtOAc $=4: 1)$ to give the target compound 27 in $79 \%$ yield $(9.5 \mathrm{mg}, 0.016 \mathrm{mmol})$. 


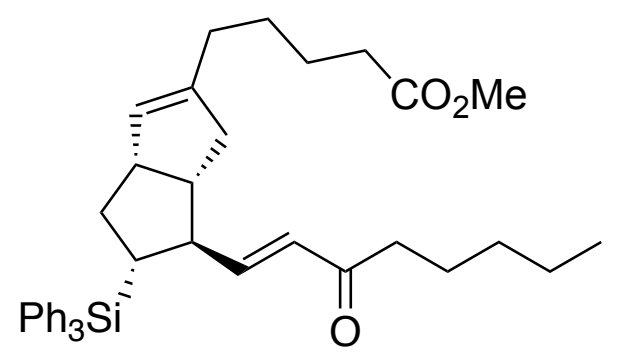

Yield: $79 \%(9.5 \mathrm{mg})$

Physical State: Colorless oil

${ }^{1}$ H NMR $\left(\mathbf{C D C l}_{3}, \mathbf{4 0 0} \mathbf{M H z}\right) \delta$ 7.50-7.63 (m, 6H), 7.36-7.40 (m, 9H), $6.45(\mathrm{dd}, 1 \mathrm{H}, J=8.0,16.0 \mathrm{~Hz}), 5.64$ $(\mathrm{d}, 1 \mathrm{H}, J=16.0 \mathrm{~Hz}), 5.10(\mathrm{~s}, 1 \mathrm{H}), 4.34($ quin, $1 \mathrm{H}, J=4.4 \mathrm{~Hz}), 4.06-4.16(\mathrm{~m}, 2 \mathrm{H}), 3.67(\mathrm{~s}, 3 \mathrm{H}), 2.27-2.42(\mathrm{~m}$, $5 \mathrm{H}), 1.78-2.04(\mathrm{~m}, 5 \mathrm{H}), 1.08-1.31(\mathrm{~m}, 15 \mathrm{H})$

${ }^{13} \mathbf{C}$ NMR (CDCl ${ }_{3}, 100$ MHz) $\delta$ 200.7, 174.1, 150.3, 141.1, 136.0, 136.0, 136.0, 136.0, 136.0, 136.0, 134.2, 134.2, 134.2, 129.4, 129.4, 129.4, 127.7, 127.7, 127.7, 127.7, 127.7, 127.7, 84.9, 53.8, 51.5, 47.2, 42.1, 40.8, $38.9,38.4,33.5,32.5,31.4,27.3,24.5,23.6,22.5,21.4,14.0$

HRMS (ESI): [M+Na] $]^{+}$calcd for $\mathrm{C}_{40} \mathrm{H}_{48} \mathrm{O}_{3} \mathrm{SiNa}$ : 627.3265, found: 627.3270

IR(neat) $v 1737,1428,1367,1109,741,702,514 \mathrm{~cm}^{-1}$

$[\alpha]_{\mathrm{D}}{ }^{26}-50.2\left(c 1.1, \mathrm{CHCl}_{3}\right)$

$\mathbf{R}_{\mathbf{f}}(n$-Hexane: EtOAc $=3: 1$, color reagent: Hanessian's stain reagent $): 0.55$

\subsection{Synthesis of aldehyde $6 \mathrm{~b}$}

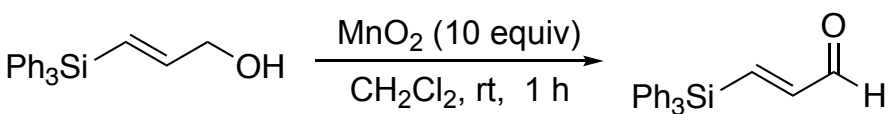

SI-11
$6 b$

To a solution of compound SI-11 ${ }^{[5]}(3.16 \mathrm{~g}, 10 \mathrm{mmol})$ in $\mathrm{CH}_{2} \mathrm{Cl}_{2}(100 \mathrm{~mL}), \mathrm{MnO}_{2}(8.6 \mathrm{~g}, 100 \mathrm{mmol})$ was added at room temperature. After stirring the reaction mixture at this temperature for $1 \mathrm{~h}$, the reaction micture was concentrated under reduced pressure. The residue was filtrated by celite ${ }^{\circledR}$, which solvent was EtOAc (5 $\times 30 \mathrm{~mL}$ ). The residue was concentrated under reduced pressure and directly purified by column chromatography on silica gel $(n$-Hexane: EtOAc $=10: 1)$ to give the target compound $\mathbf{6 b}(2.7 \mathrm{~g}, 8.7 \mathrm{mmol})$ in $87 \%$ yield.

(E)-3-(Triphenylsilyl)acrylaldehyde (6b)<smiles>O=C/C=C/[SbH2]</smiles>

Yield: $87 \%(2.7 \mathrm{~g})$

Physical State: White amorphous

${ }^{1} \mathbf{H}$ NMR $\left(\mathbf{C D C l}_{3}, \mathbf{4 0 0}\right.$ MHz) $\delta 9.66(\mathrm{~d}, 1 \mathrm{H}, J=7.6), 7.66(\mathrm{~d}, 1 \mathrm{H}, J=18.4 \mathrm{~Hz}), 7.51-7.53(\mathrm{~m}, 6 \mathrm{H}), 7.45-7.50$ $(\mathrm{m}, 3 \mathrm{H}), 7.39-7.43(\mathrm{~m}, 6 \mathrm{H}), 6.59(\mathrm{dd}, 1 \mathrm{H}, J=7.6,18.4 \mathrm{~Hz})$ 
${ }^{13} \mathbf{C} \mathbf{N M R}\left(\mathbf{C D C l}_{3}, \mathbf{1 0 0} \mathbf{M H z}\right) \delta 194.3,152.3,147.7,135.9,135.9,135.9,135.9,135.9,135.9,132.0,132.0$, $132.0,130.3,130.3,130.3,128.2,128.2,128.2,128.2,128.2,128.2$

HRMS (ESI): $[\mathrm{M}+\mathrm{Na}]^{+}$calcd for $\mathrm{C}_{21} \mathrm{H}_{18} \mathrm{OSiNa}$ : 337.1019, found: 337.1019

IR(neat) $v 1683,1428,1113,740,700,548 \mathrm{~cm}^{-1}$

$\mathbf{R}_{\mathbf{f}}(n$-Hexane: EtOAc $=3: 1$, color reagent: Hanessian's stain reagent $): 0.30$

\subsection{Information of compound 11}

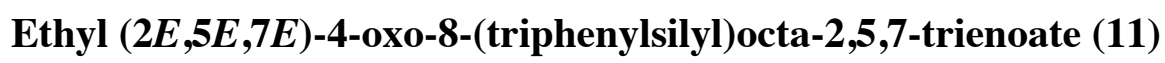<smiles>CCOC(=O)C=CC(=O)C=CC=C[SiH2]c1ccccc1</smiles>

Yield: 30\% (19.7 mg) (Table 1, entry 4)

Physical State: yellow oil

${ }^{1} \mathbf{H}$ NMR $\left(\mathbf{C D C l}_{3}, 400 \mathrm{MHz}\right) \delta$ 7.64-7.66 (m, 2H), 7.53-7.55 (m, 5H), 7.35-7.49 (m, $\left.10 \mathrm{H}\right), 7.03(\mathrm{~d}, 1 \mathrm{H}, J=$ $18.0 \mathrm{~Hz}), 6.76(\mathrm{dd}, 1 \mathrm{H}, J=10.8,18.4 \mathrm{~Hz}), 6.74(\mathrm{~d}, 1 \mathrm{H}, J=15.6 \mathrm{~Hz}), 6.37(\mathrm{~d}, 1 \mathrm{H}, J=15.6 \mathrm{~Hz}), 4.27(\mathrm{q}, 2 \mathrm{H}$, $J=7.2 \mathrm{~Hz}), 1.33(\mathrm{t}, 3 \mathrm{H}, J=7.2 \mathrm{~Hz})$,

${ }^{13} \mathbf{C}$ NMR $\left(\mathbf{C D C l}_{3}, \mathbf{1 0 0}\right.$ MHz) $\delta 188.9,165.5,146.0,141.7,138.2,135.9,135.9,135.9,135.9,135.9,135.9$, $135.0,135.0,135.0,133.2,130.0,129.9,128.0,128.0,128.0,128.0,128.0,128.0,127.9,127.9,127.9,61.3$, 14.1

HRMS (ESI): $[\mathrm{M}+\mathrm{Na}]^{+}$calcd for $\mathrm{C}_{28} \mathrm{H}_{26} \mathrm{O}_{3} \mathrm{SiNa}: 461.1543$, found: 461.1543

$\operatorname{IR}($ neat $) v 3069,1722,1662,1597,1428,1368,1303,1184,909,781,701 \mathrm{~cm}^{-1}$

$\mathbf{R}_{\mathbf{f}}(n$-Hexane: EtOAc $=3: 1$, color reagent: Hanessian's stain reagent $): 0.70$

\section{References}

[1] Umekubo, N.; Rohei, I.; Hayashi, Y. One-pot Synthesis of Chiral cis-Hydrindanes via Diphenylprolinol Silyl Ether Mediated Domino Reaction and Aldol Condensation. Chem. Lett. 2020, 49, 867-869.

[2] Umekubo, N.; Suga, Y.; Hayashi, Y. Pot and time economies in the total synthesis of Corey lactone. Chem. Sci. 2020, 11, 1205-1210.

[3] Umemiya, S.; Hayashi, Y. Pot Economy in the Synthesis of Prostaglandin $\mathrm{A}_{1}$ and $\mathrm{E}_{1}$ Methyl Esters. Angew. Chem. Int. Ed., 2013, 52, 3450 .

[4] (a) Nagy, E. E.; Hyatt, I. F. D.; Gettys, K. E.; Yeazell, S. T.; Frempong, Jr. S. K.; Croatt, J.-M. P. Sequential Pd(0)-, Rh(I)-, and Ru(II)-Catalyzed Re-actions in a Nine-Step Synthesis of Clinprost. Org. Lett. 2013, 15, 586-589. (b) Ogawa, Y.; Shibasaki, M. The intramolecular thermal ene reaction route to (+)-9(O)-methano$\Delta^{6(9 \alpha)}-\mathrm{PGI}_{1}$. Tetrahedron Lett. 1984, 25, 1067-1070.

[5] Murphy, P. I.; Spencer, J. L.; Procter, G. Allylsilanes in organic synthesis; convenient preparation of synthetic intermediates by catalytic hydrosilation of acetylenic alcohols. Tetrahedron Lett., 1990, 31, 1051. 


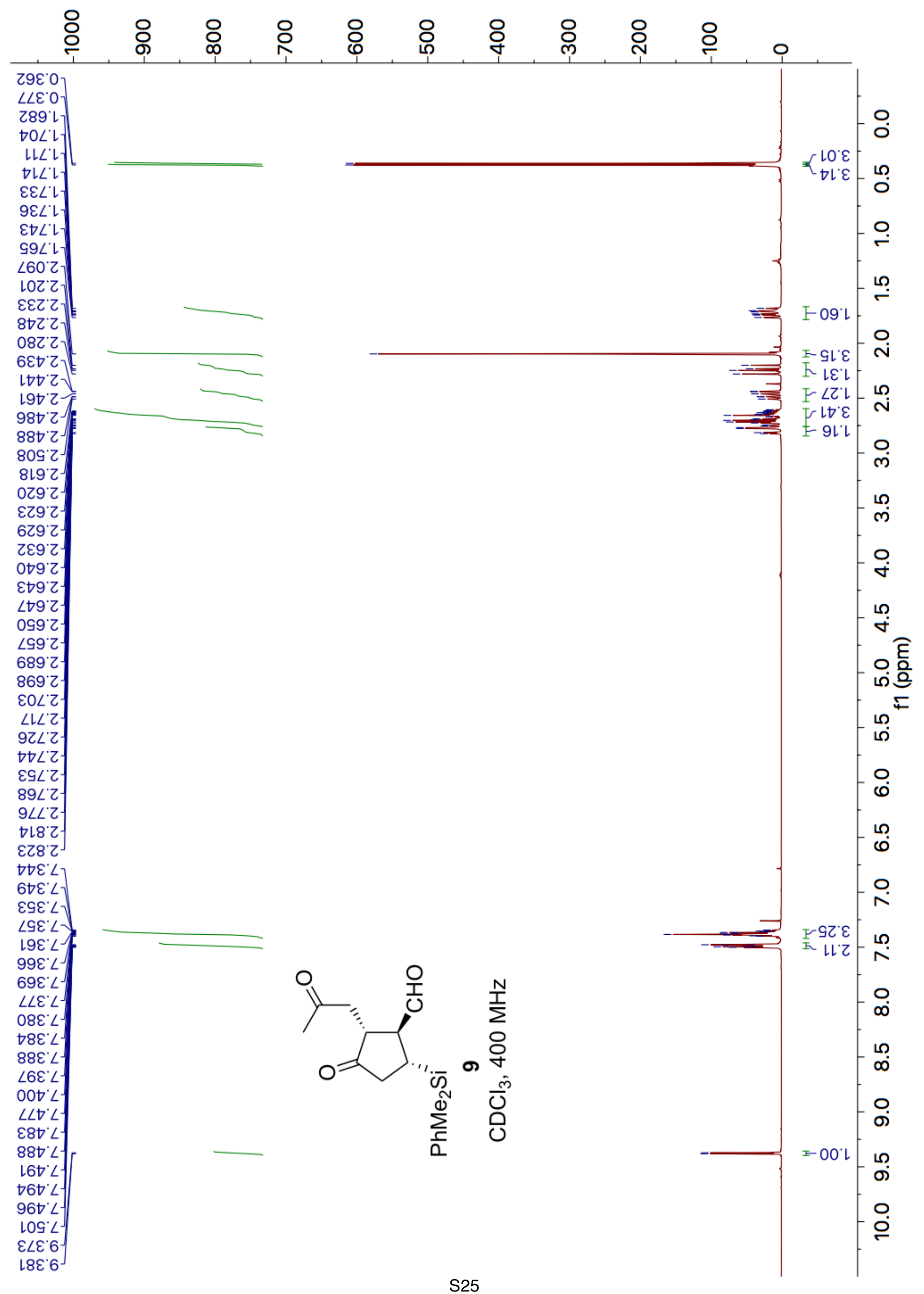




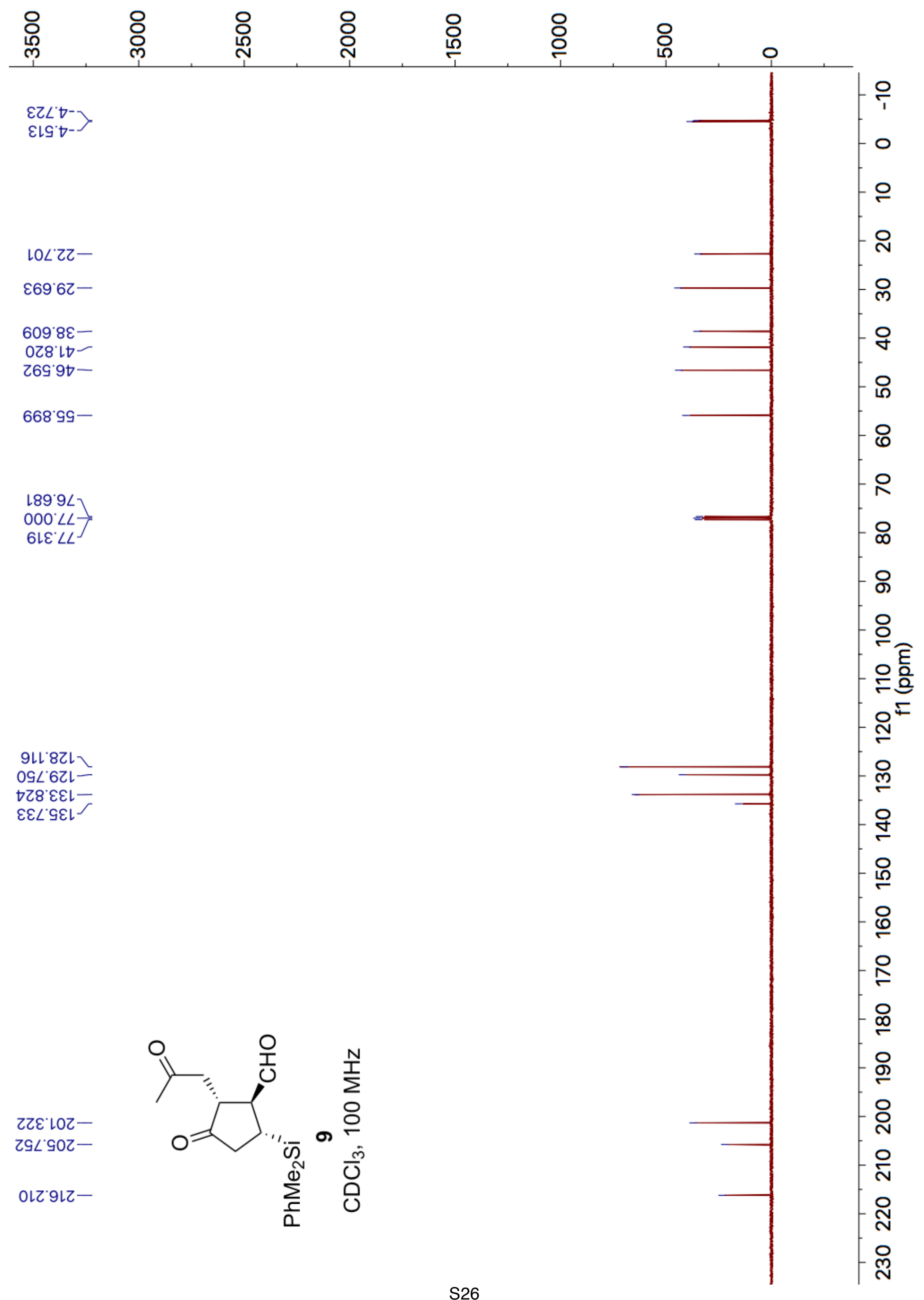




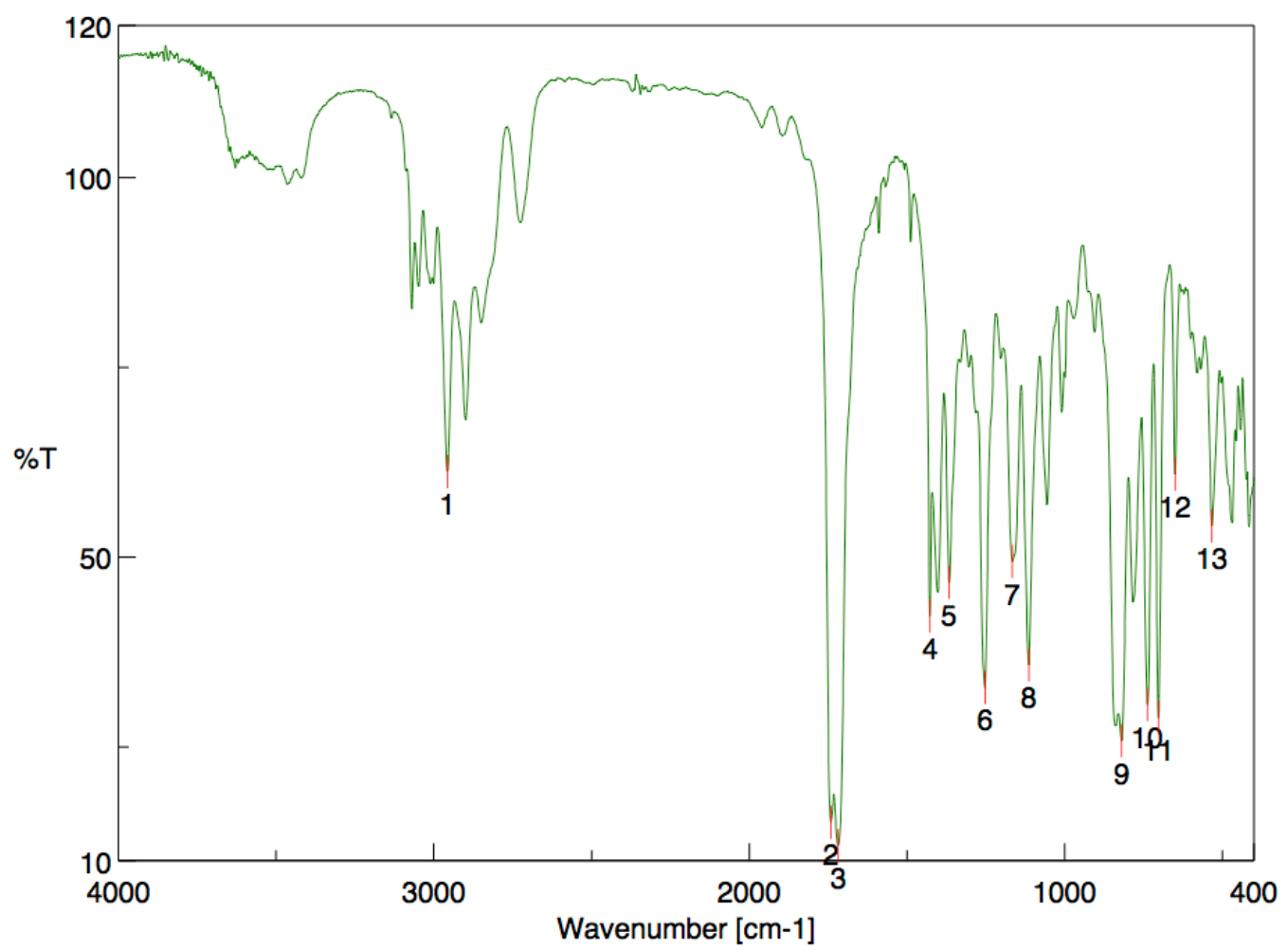

\begin{tabular}{llllll}
\multicolumn{2}{l}{ [ピーク検出結果 ] } & & & & \\
No. & 位置 & 強度 & No. & 位置 & 強度 \\
1 & 2956.34 & 61.2413 & 2 & 1741.41 & 15.058 \\
3 & 1718.26 & 11.9538 & 4 & 1427.07 & 42.2042 \\
5 & 1366.32 & 46.6522 & 6 & 1252.54 & 32.8038 \\
7 & 1165.76 & 49.3905 & 8 & 1113.69 & 35.7869 \\
9 & 819.598 & 25.7743 & 10 & 737.639 & 30.5363 \\
11 & 702.926 & 28.7953 & 12 & 649.893 & 60.8944 \\
13 & 533.221 & 54.0602 & & &
\end{tabular}<smiles>CC(=O)C[C@H]1C(=O)C[C@H](C)[C@@H]1C=O</smiles>

$\mathrm{PhMe}_{2} \mathrm{Si}$

\section{9}




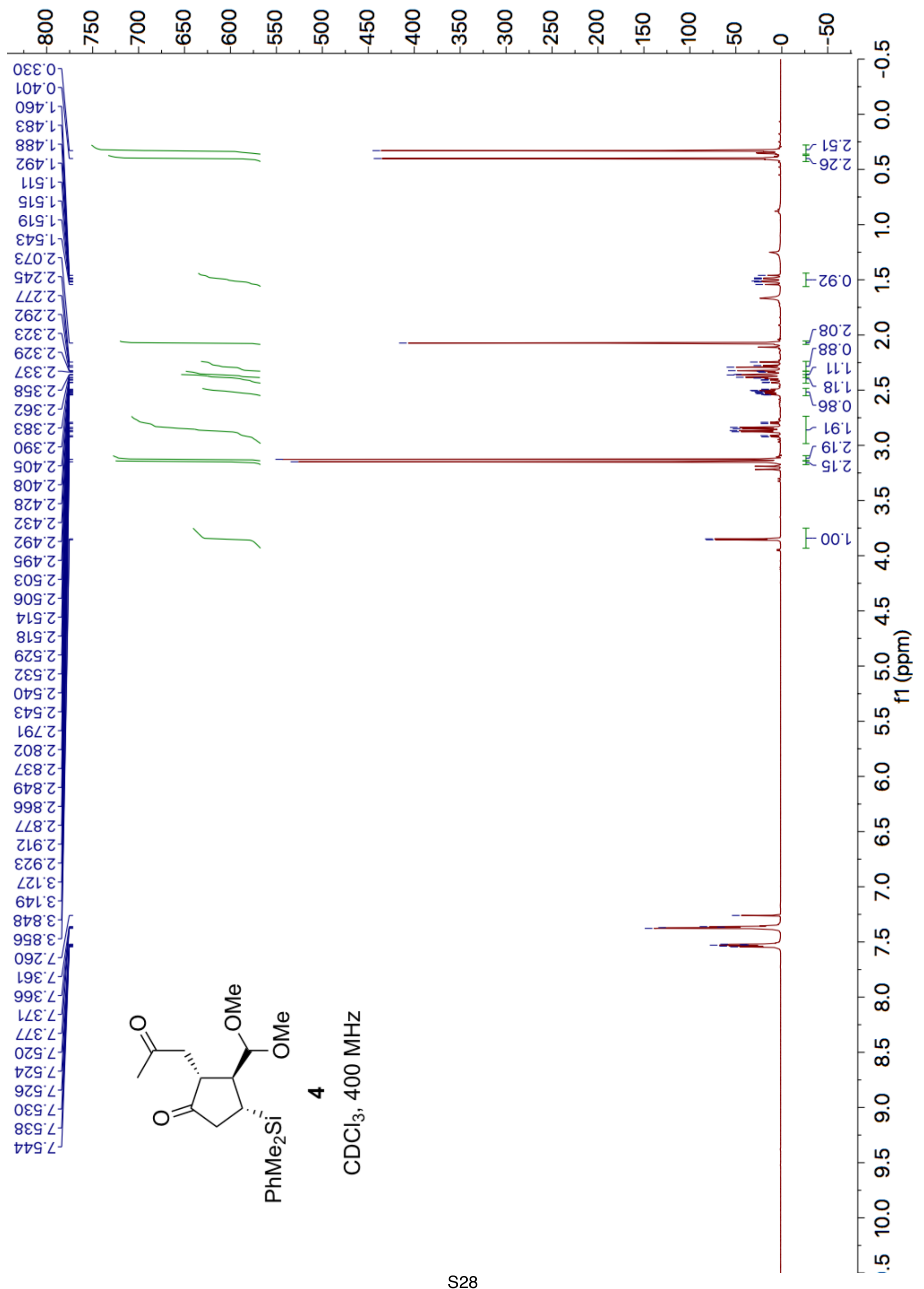




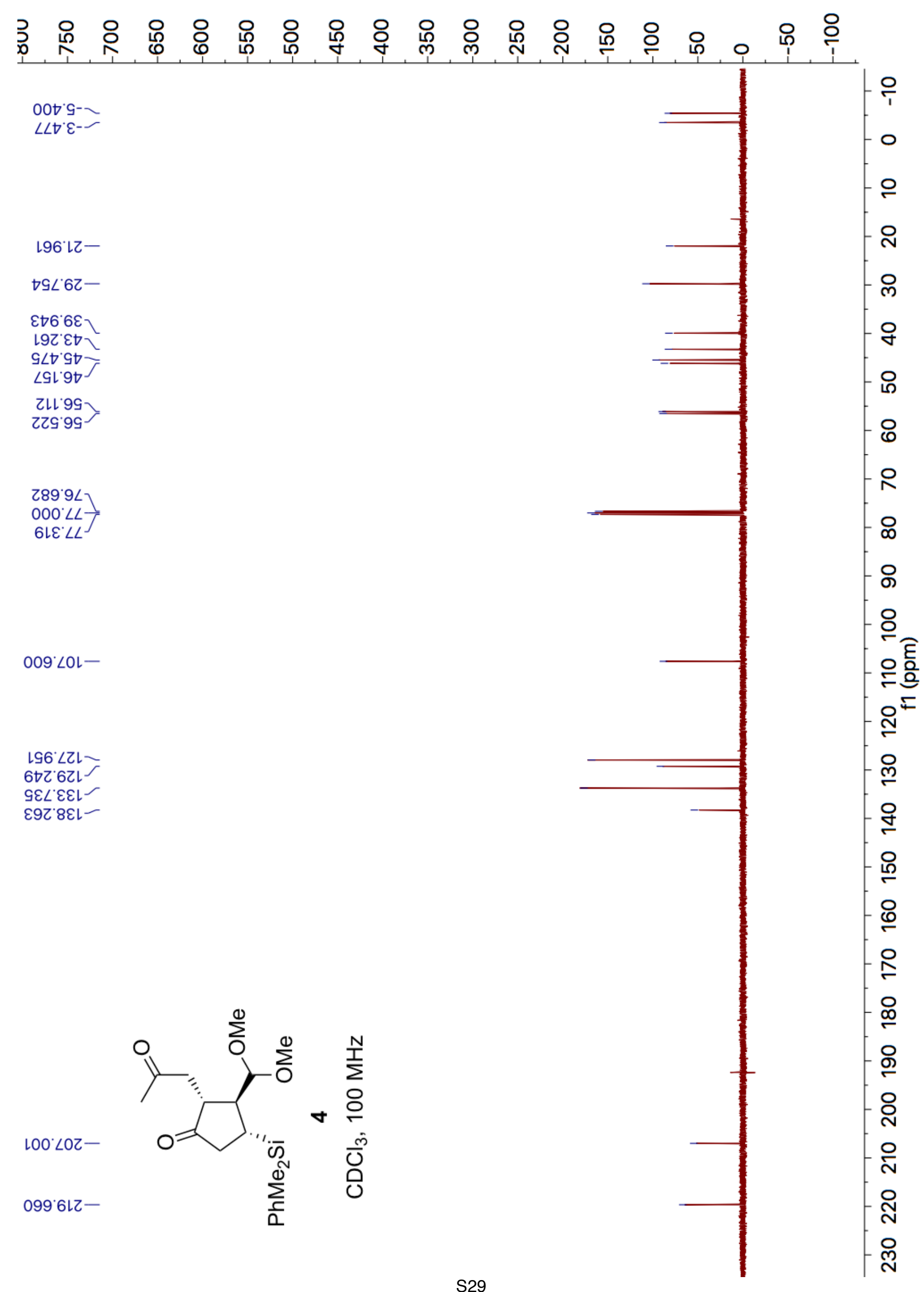




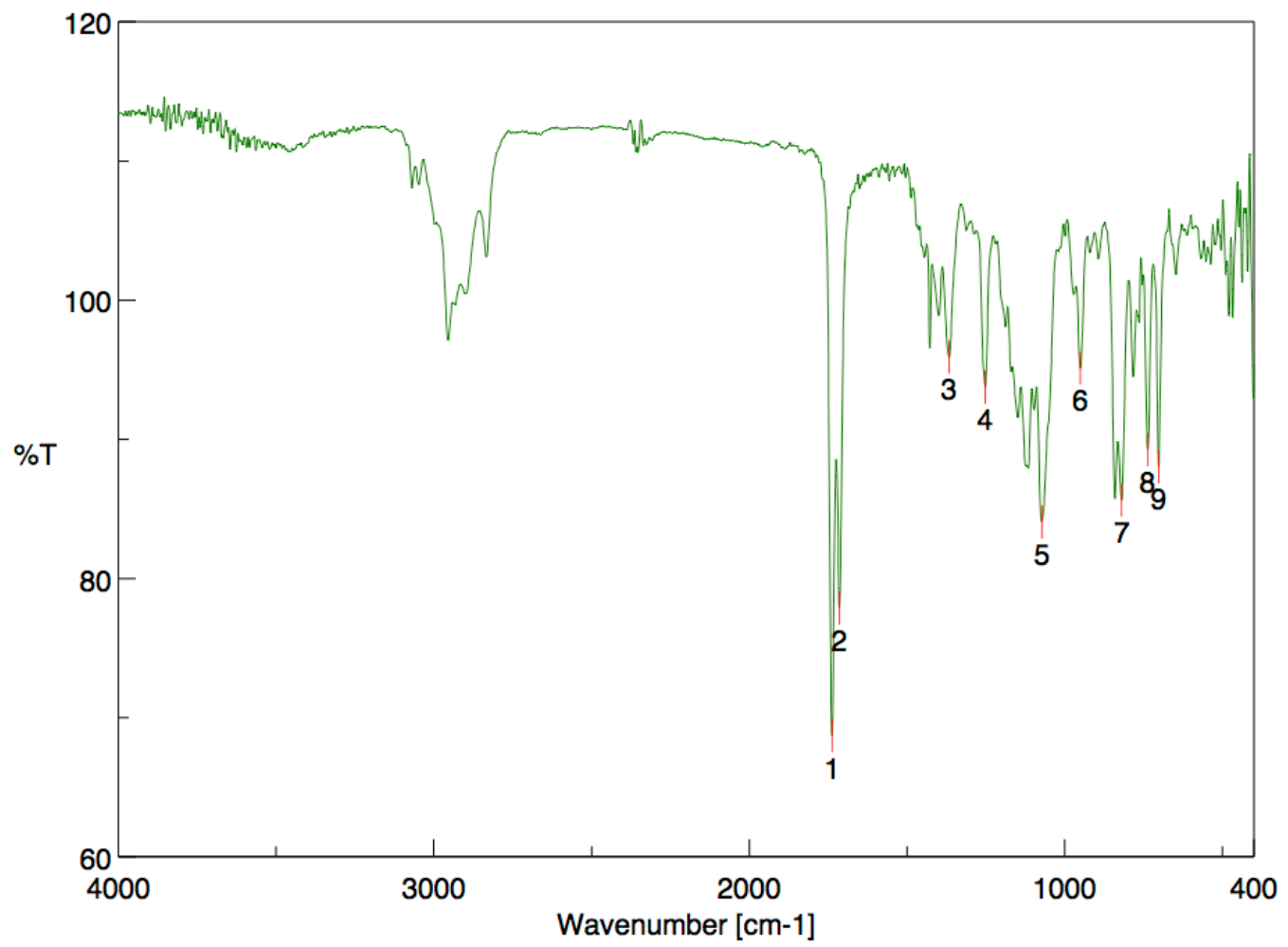

\begin{tabular}{|c|c|c|c|c|c|}
\hline \multicolumn{6}{|c|}{ [ ピーク検出結果 ] } \\
\hline No. & 位置 & 強度 & No. & 位置 & 強度 \\
\hline 1 & 1737.55 & 68.706 & 2 & 1714.41 & 77.8652 \\
\hline 3 & 1366.32 & 95.9023 & 4 & 1251.58 & 93.7251 \\
\hline 5 & 1073.19 & 84.0376 & 6 & 950.734 & 95.1095 \\
\hline 7 & 819.598 & 85.6203 & 8 & 736.674 & 89.2426 \\
\hline 9 & 701.962 & 88.0059 & & & \\
\hline
\end{tabular}<smiles>CO[SiH2][C@@H]1CC(=O)[C@H](CC(C)=O)[C@@H]1C(OC)OC</smiles> 


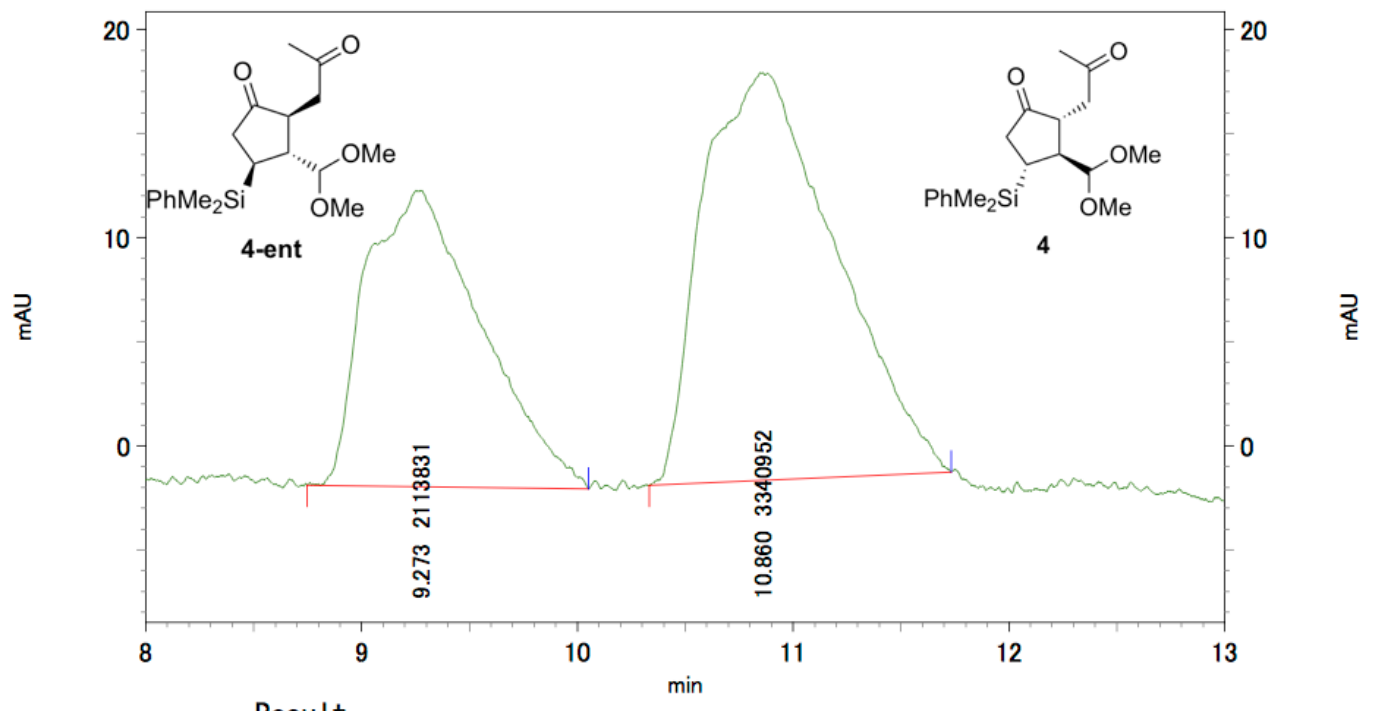

1: $214 \mathrm{~nm}, 4$ nm結果

Pk \# Retention time / min

Integration/\%

10.860

38.752
61.248

\begin{tabular}{|r|r|r|}
\hline$r-$ s l & 100.000 \\
\hline
\end{tabular} 


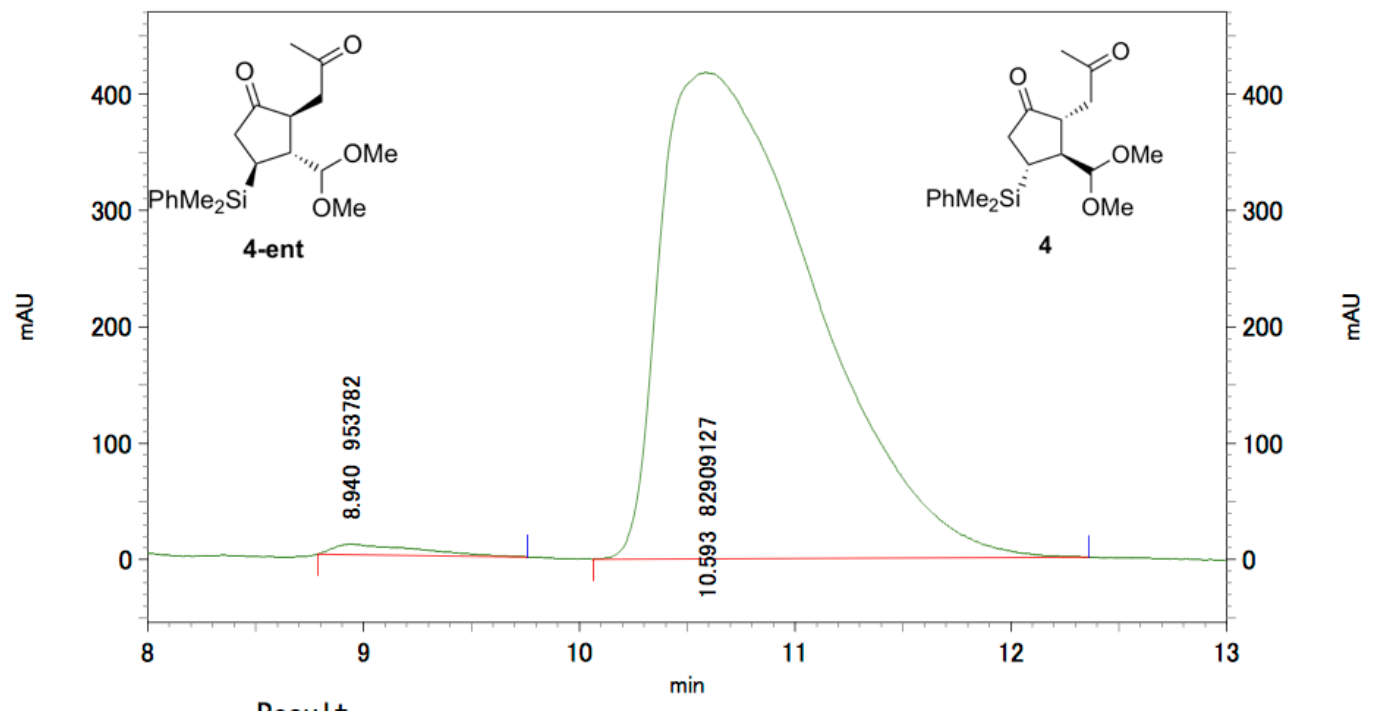

1: $214 \mathrm{~nm}, 4 \mathrm{~nm}$ 結果

Pk \# $\quad$ Retention time / min

Integration/\%

10.593

98.863

\begin{tabular}{|r|r|r|}
\hline$r-$ gll & 100.000 \\
\hline
\end{tabular}




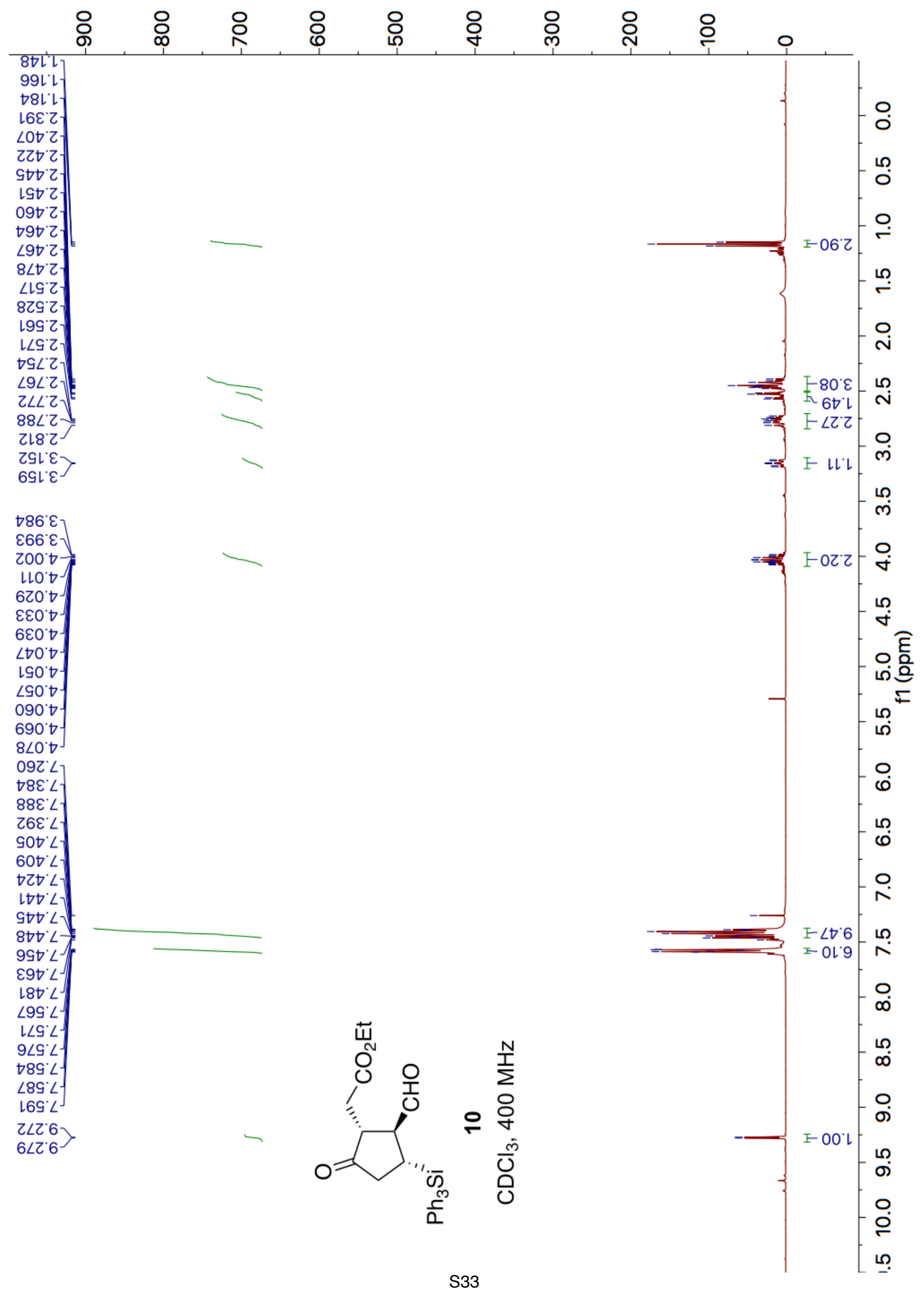




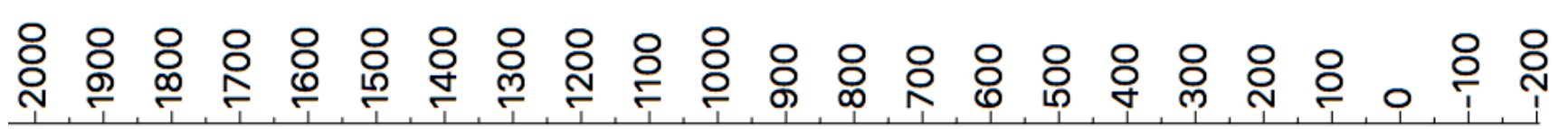

て00ナレー

เ8LレZー

$\angle L G \cdot \varepsilon^{\int}$

$\nabla 0 \angle{ }^{\circ} 6 \varepsilon-$

$0 Z L^{\circ} \angle \nabla \backslash$

$\angle S L G S-$

$0 \angle 8^{\circ} 09-$

$289^{\circ} 9 /$

$000 \angle L T$

$6 L \varepsilon^{\prime} L L$

$\angle 0 \varepsilon^{\prime} 8 Z \mathrm{~L}$

GE乙 OعL-

99l乙EL

ع96.9عا

99l:LLL-

乙29.00乙-

カレOGLZー

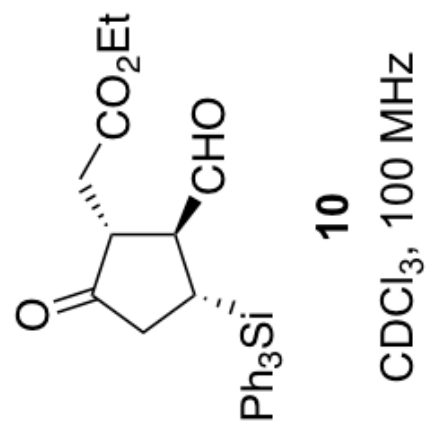

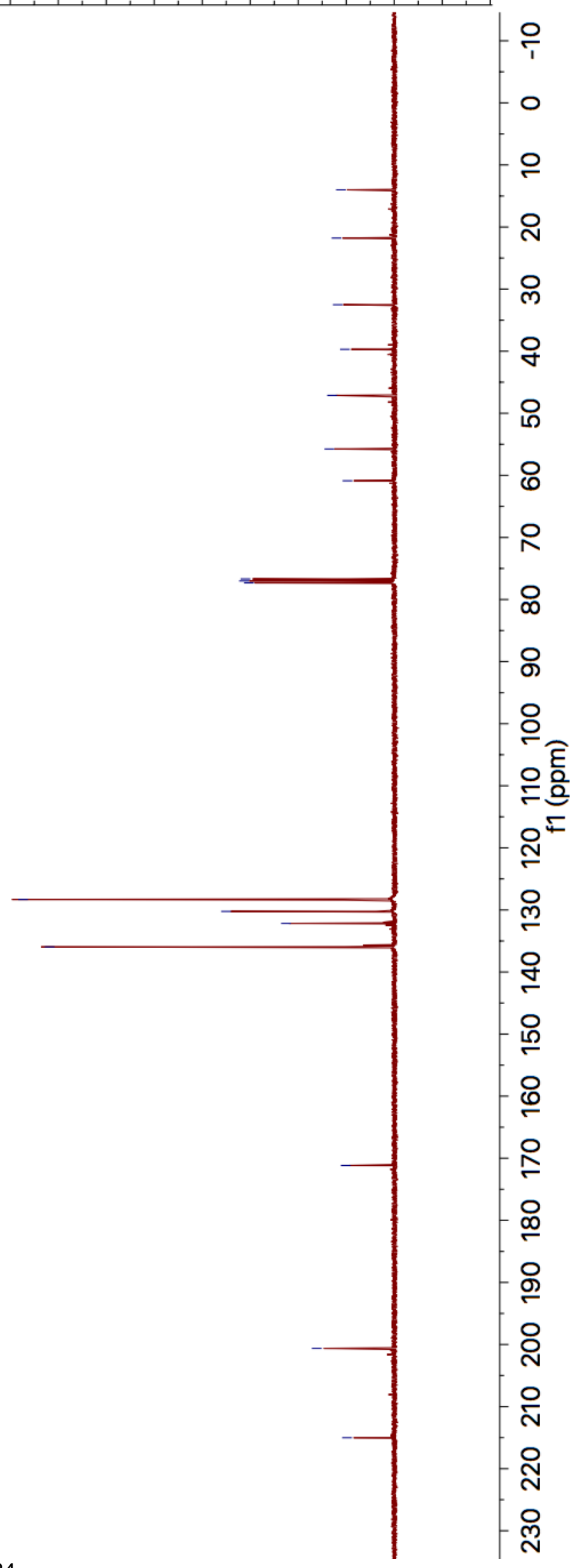




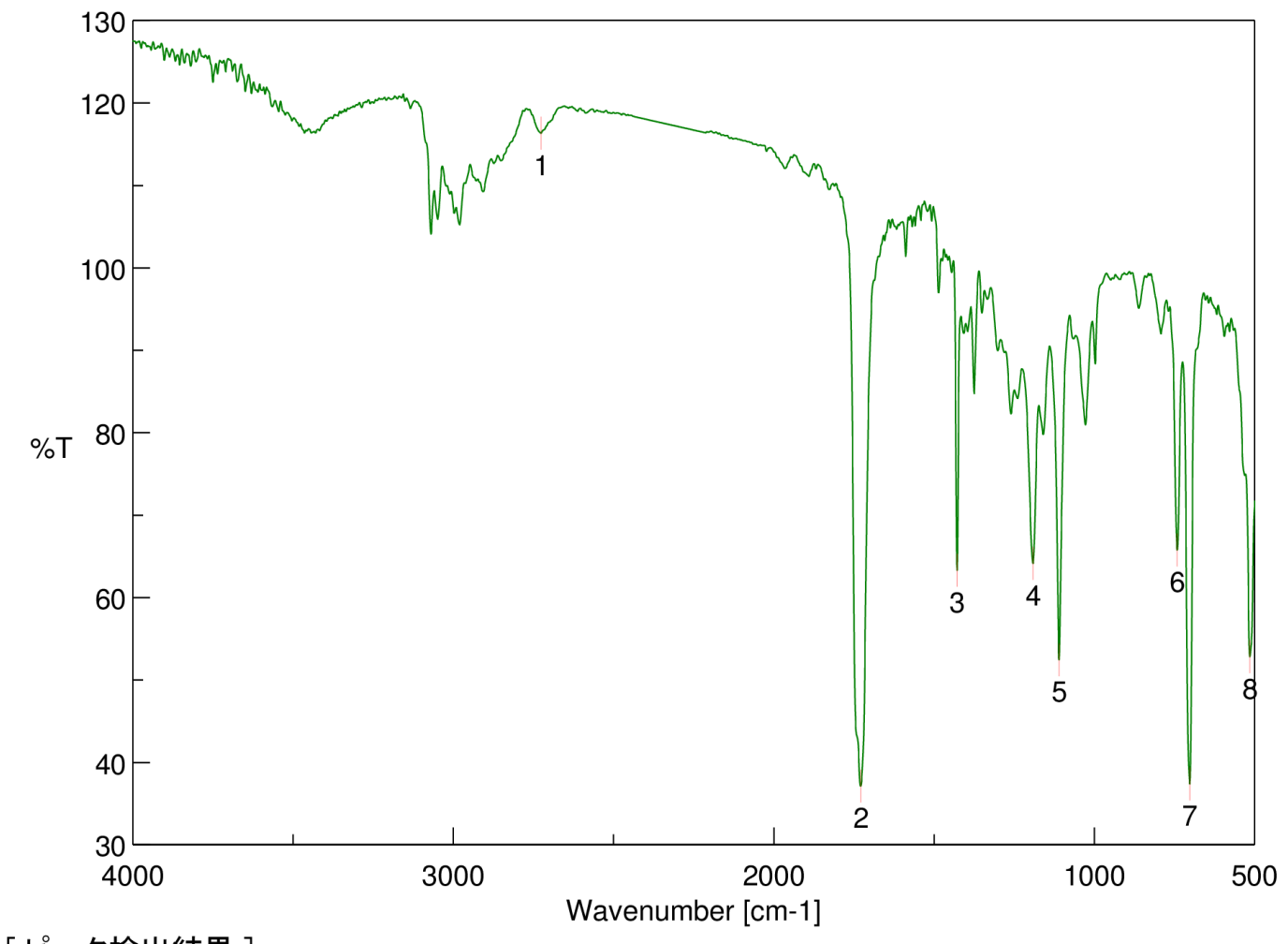

[ピーク検出結果 ]

$\begin{array}{llllll}\text { No. } & \text { 位置 } & \text { 強度 } & \text { No. } & \text { 位置 } & \text { 強度 } \\ 1 & 2725.89 & 116.327 & 2 & 1728.87 & 37.1229 \\ 3 & 1428.03 & 63.2978 & 4 & 1191.79 & 64.1286 \\ 5 & 1109.83 & 52.4138 & 6 & 741.496 & 65.7588 \\ 7 & 701.962 & 37.3836 & 8 & 514.901 & 52.823\end{array}$

$\sum_{\mathrm{CHO}} \mathrm{CO}_{2} \mathrm{Et}$

$\mathrm{Ph}_{3} \mathrm{Si}^{-}$

10 


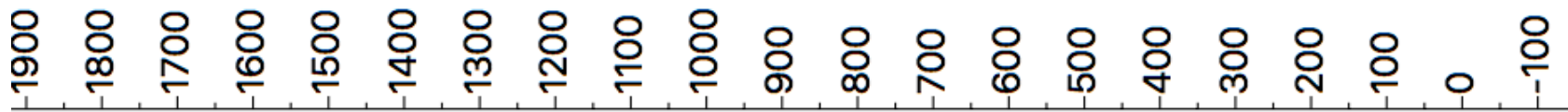

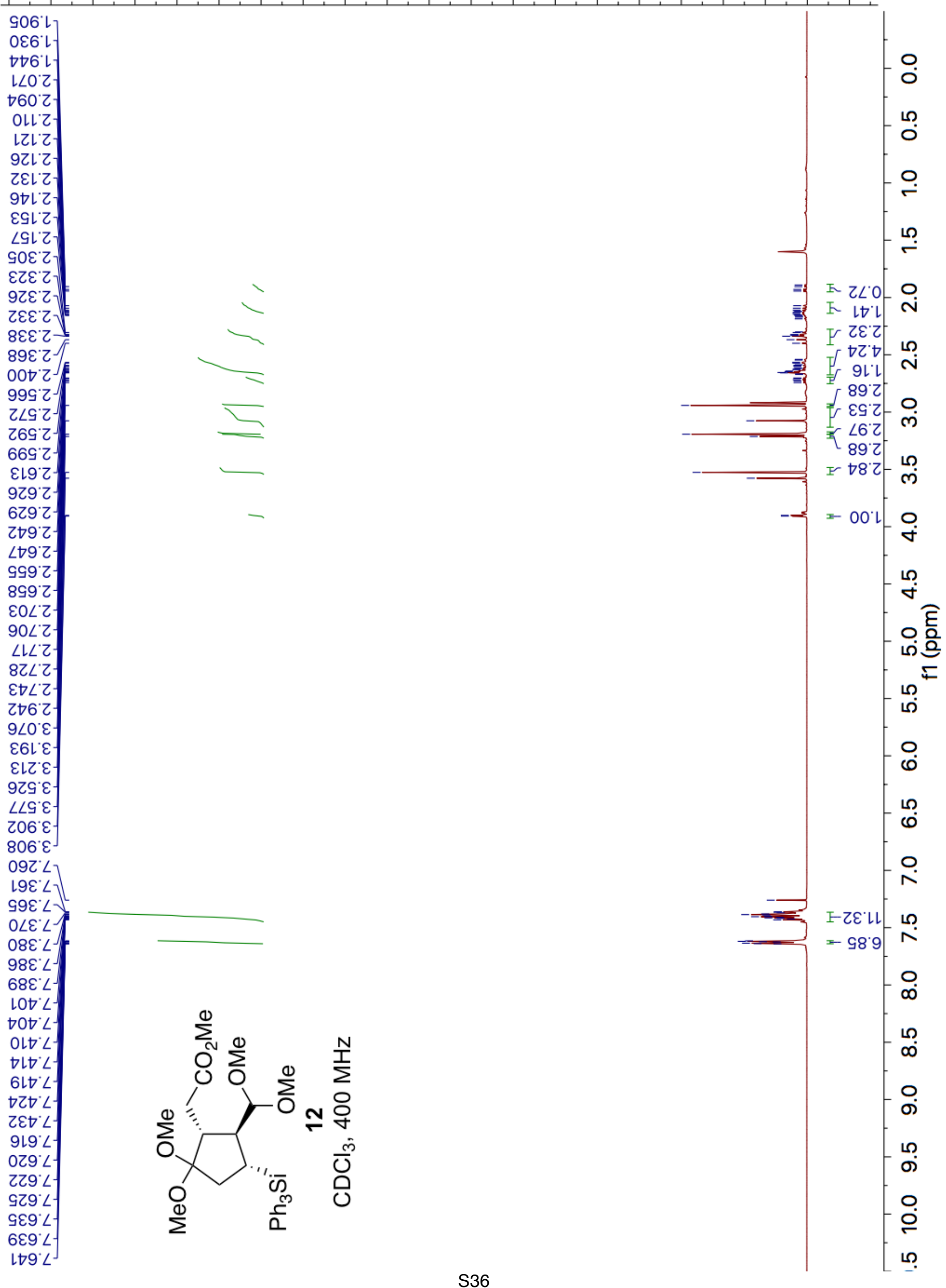




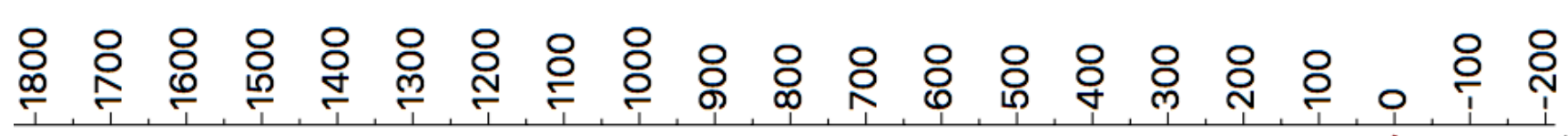

Zてヤ6レー

8LL'E\&

$\nabla \varepsilon\llcorner\cdot\llcorner\nabla$

LG9'Gt

\&86.97]

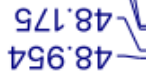

Z6E'เS

OSL'GST

286.99

$189.9 /$

$000 \angle L \frac{Y}{5}$

$81 \varepsilon^{\prime} \angle L$

89t $90 \mathrm{~L}$

$9 \nabla t \mathrm{LOL}^{\circ}$

$7900^{\circ} 8 \mathrm{~V}$ 乙

ISL'6ZL-

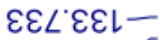

เG6'GEL

งカザてレー

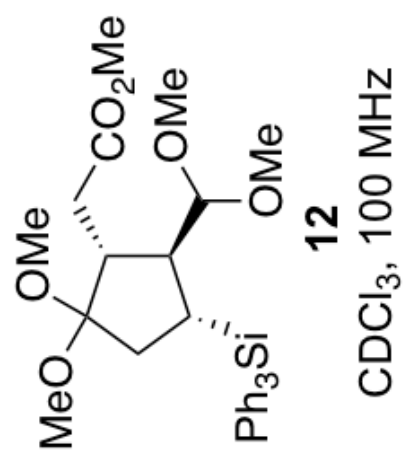

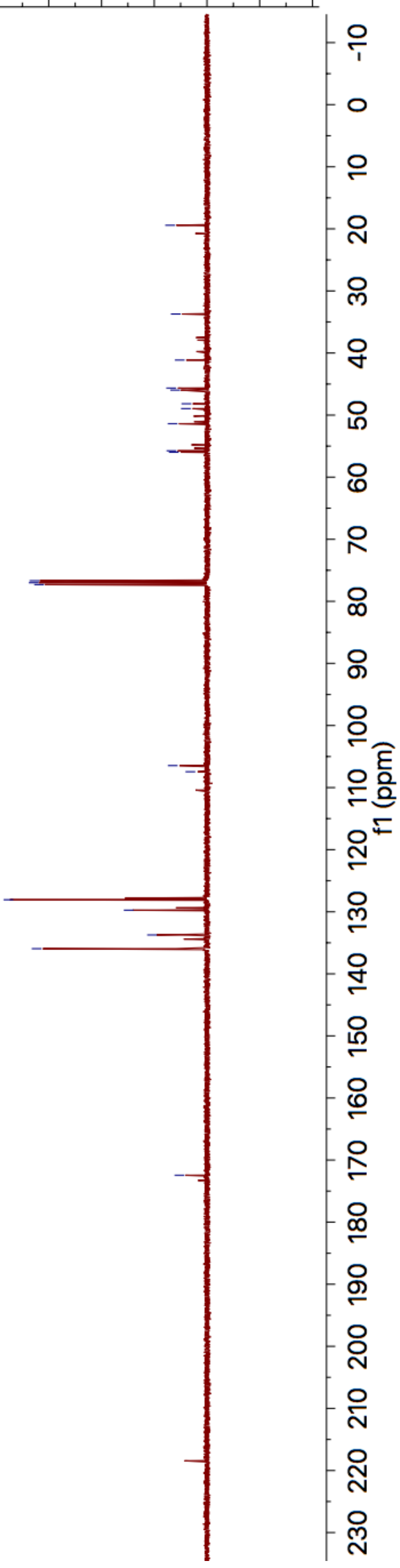




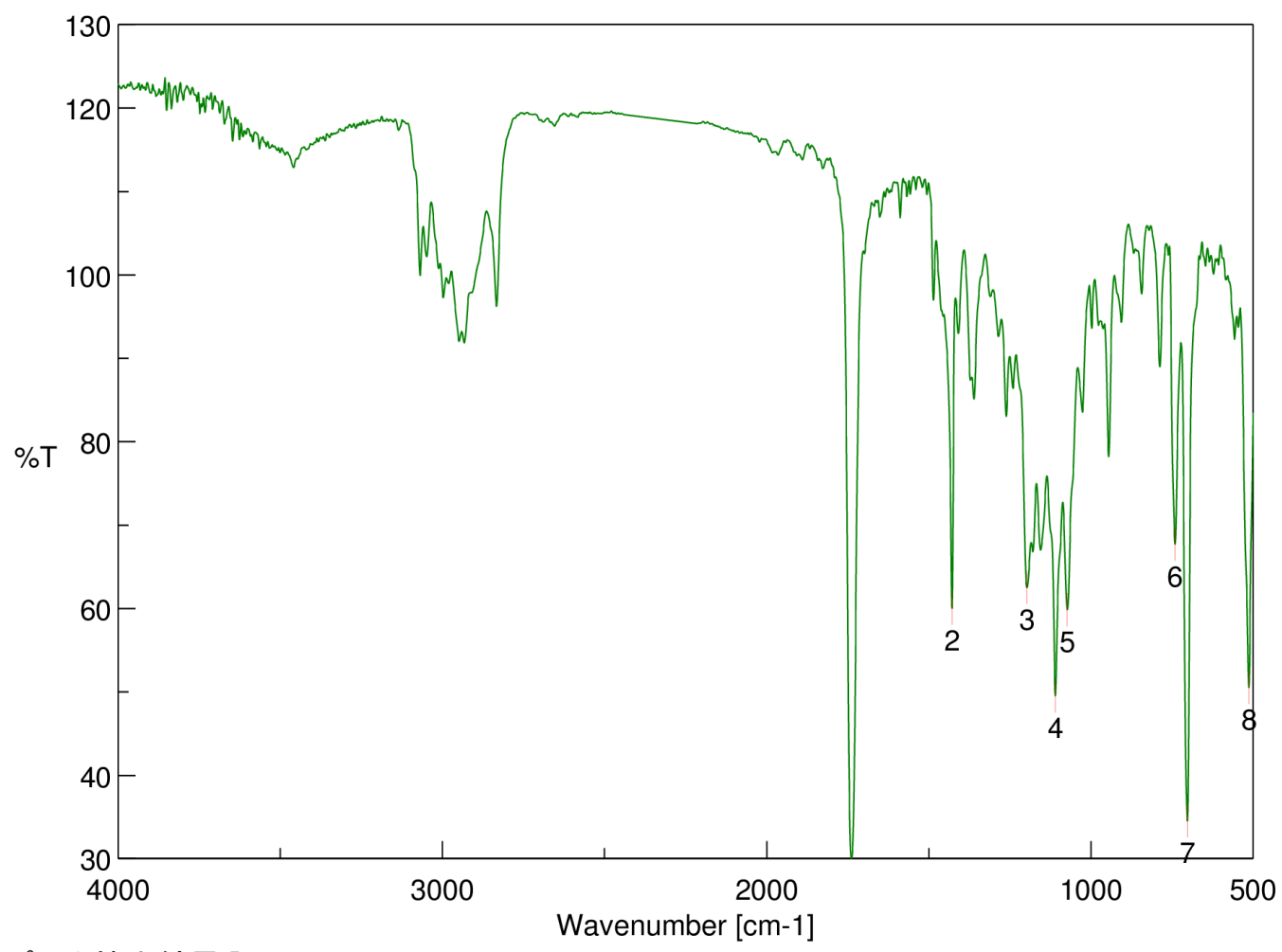

[ピーク検出結果 ]

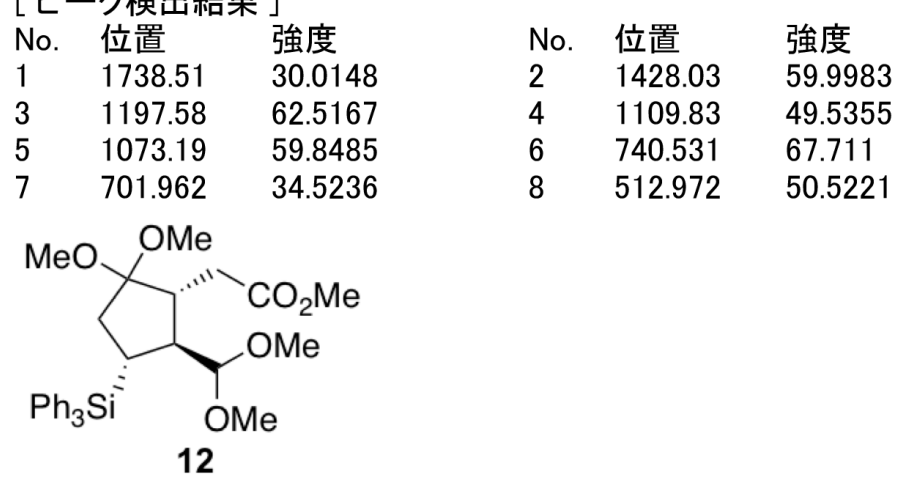




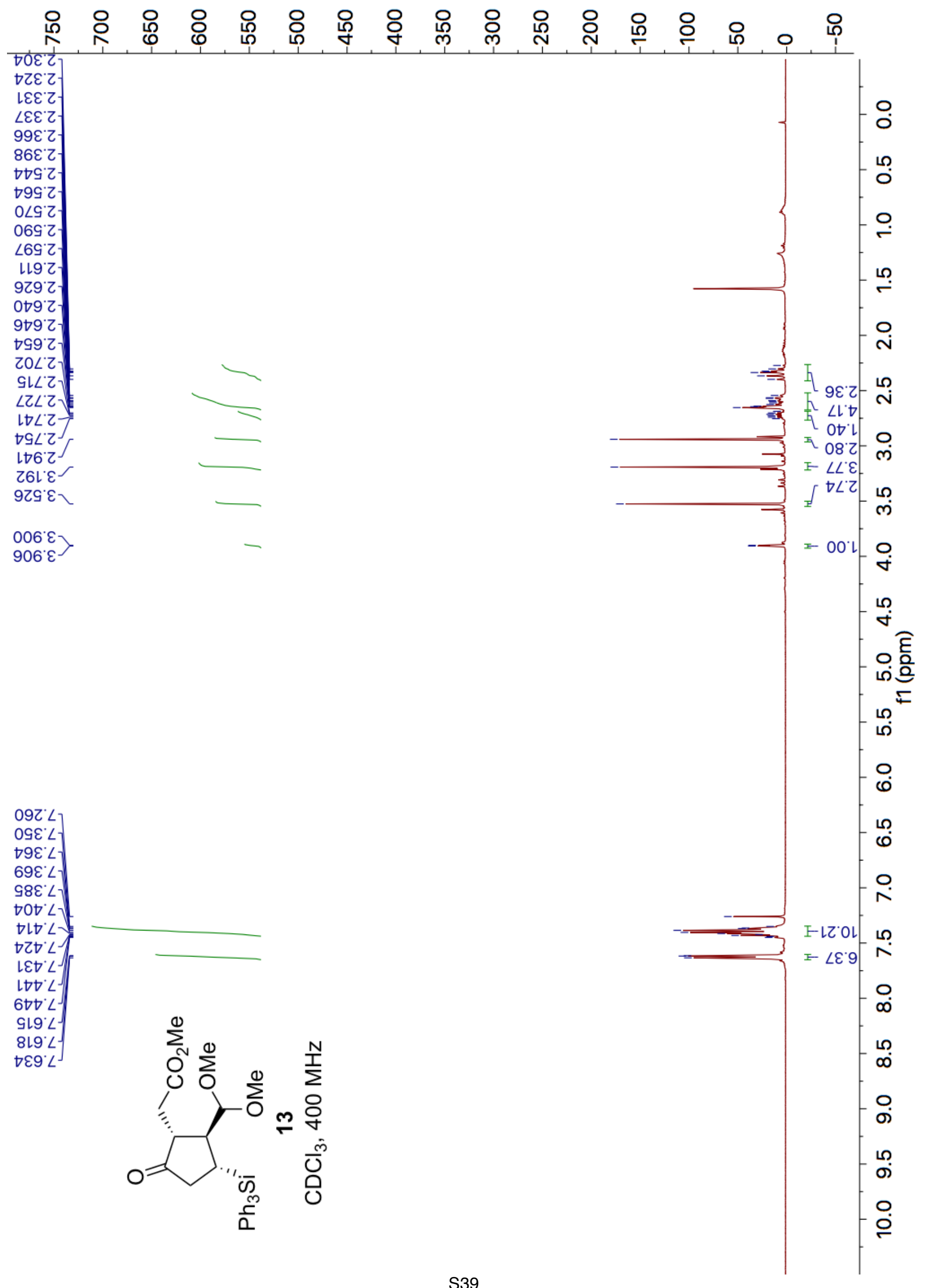




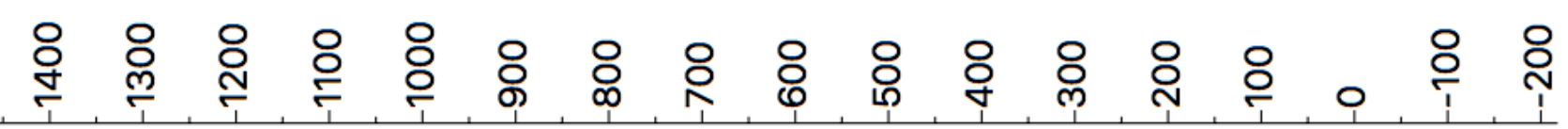

$\angle Z \nabla 6 L-$

$\nabla 乙 L \mathcal{E \varepsilon - ~}$

6ยl'Lt

$\left.899^{\circ} 97\right]^{2}$

$\angle 6 \varepsilon^{\circ}$ '

9GL'G9

886.99

$289^{\circ} 9 L$

$000 \angle L \frac{1}{5}$

$6 L \varepsilon^{\prime} \angle L$

$\triangleright 9 \nabla^{\circ} 90 L-$

6908ZL

GSL6ZL-

$0 \nabla L ' \varepsilon \varepsilon\llcorner-$

996'งع/

6カサてடLー

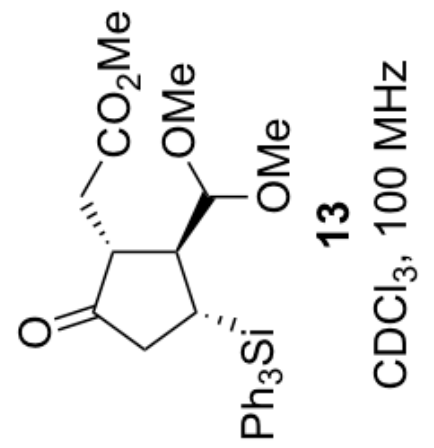

งカ78เZ- 


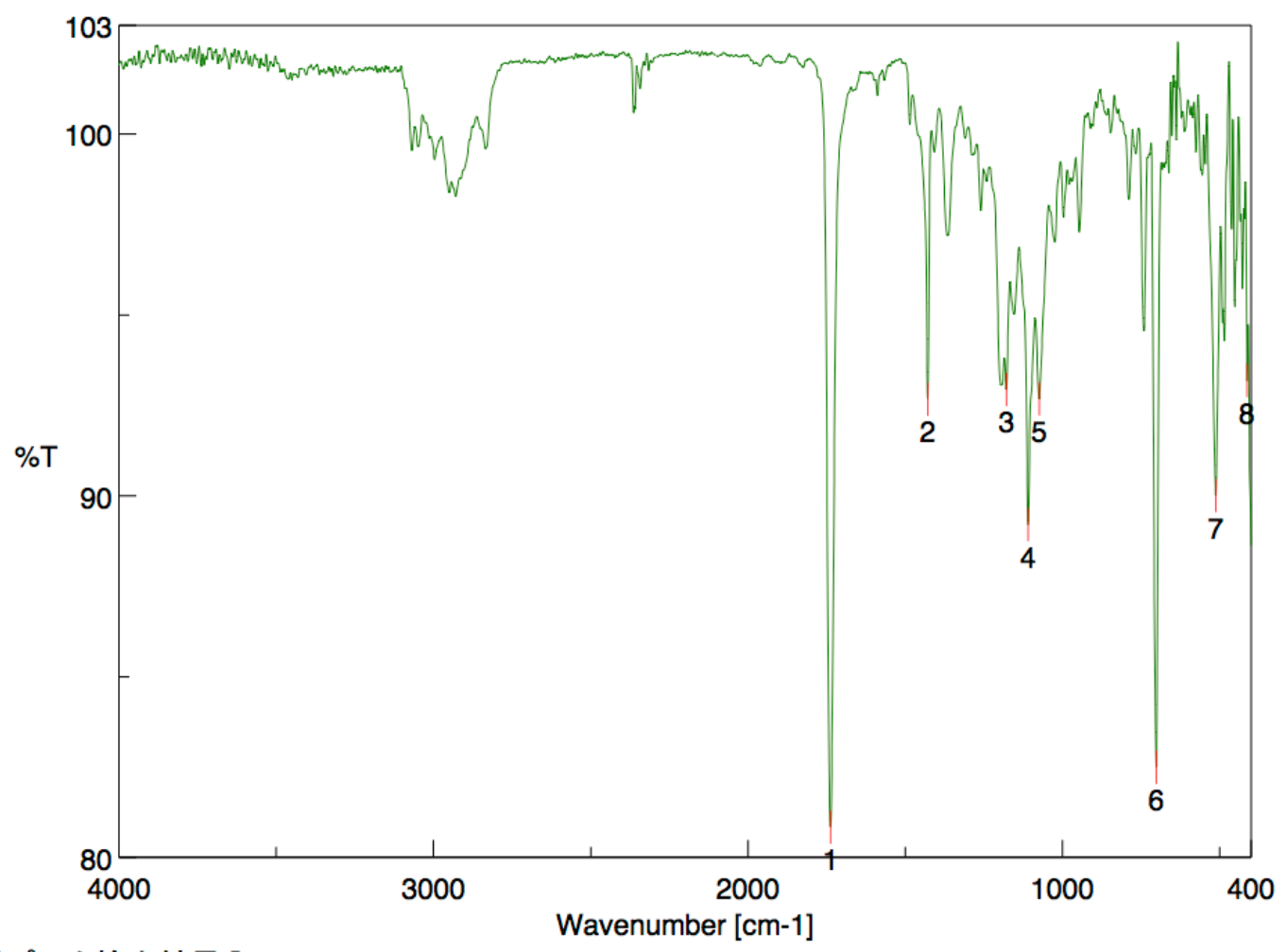

[ピーク検出結果 ]

$\begin{array}{llllll}\text { No. } & \text { 位置 } & \text { 強度 } & \text { No. } & \text { 位置 } & \text { 強度 } \\ 1 & 1737.55 & 80.8339 & 2 & 1428.03 & 92.6639 \\ 3 & 1178.29 & 92.9281 & 4 & 1108.87 & 89.1996 \\ 5 & 1074.16 & 92.6711 & 6 & 701.962 & 82.4883 \\ 7 & 512.008 & 90.0063 & 8 & 412.692 & 93.1772\end{array}$<smiles>COC(OC)[C@H]1[C@@H](CC(C)=O)CC(=O)[C@H]1[SiH2]c1ccccc1</smiles>

13 


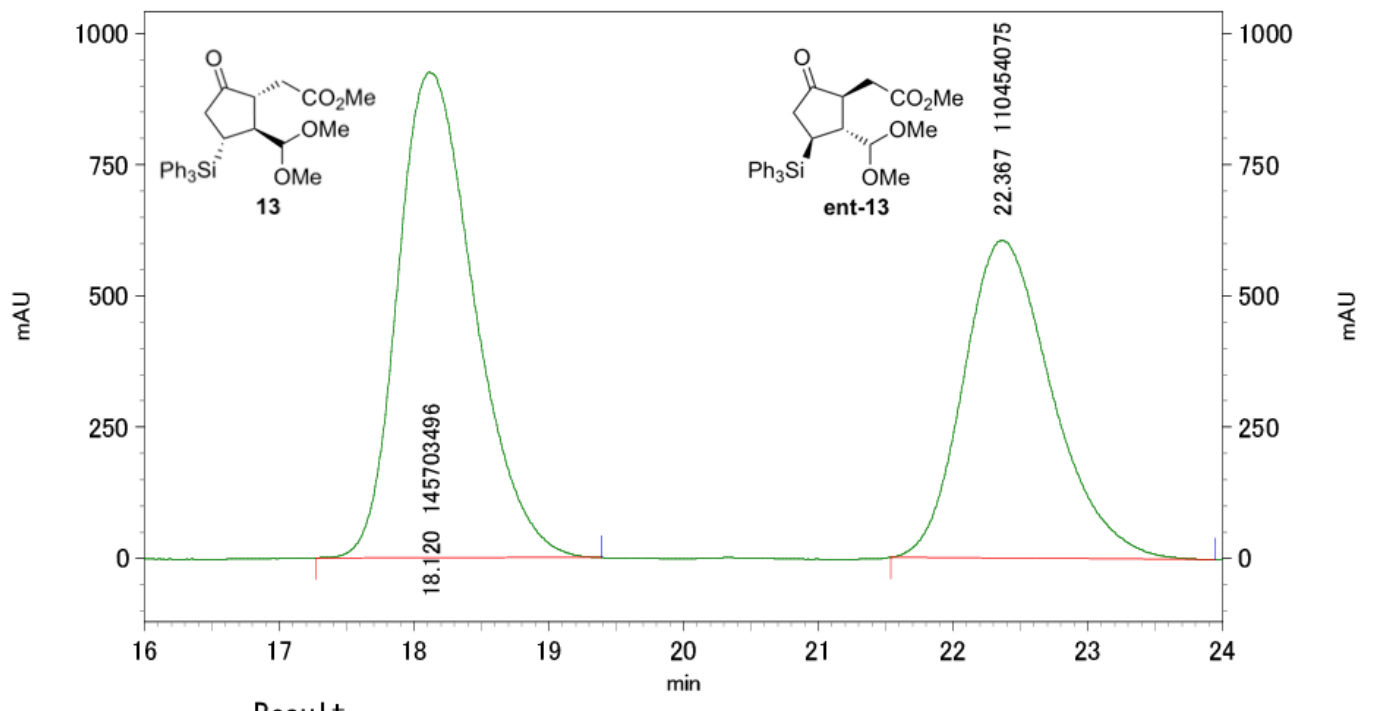

Result

1: $214 \mathrm{~nm}, 4$ nm結果

\begin{tabular}{|r|r|r|}
\hline$r-$ 舛 & 100.000 \\
\hline
\end{tabular}




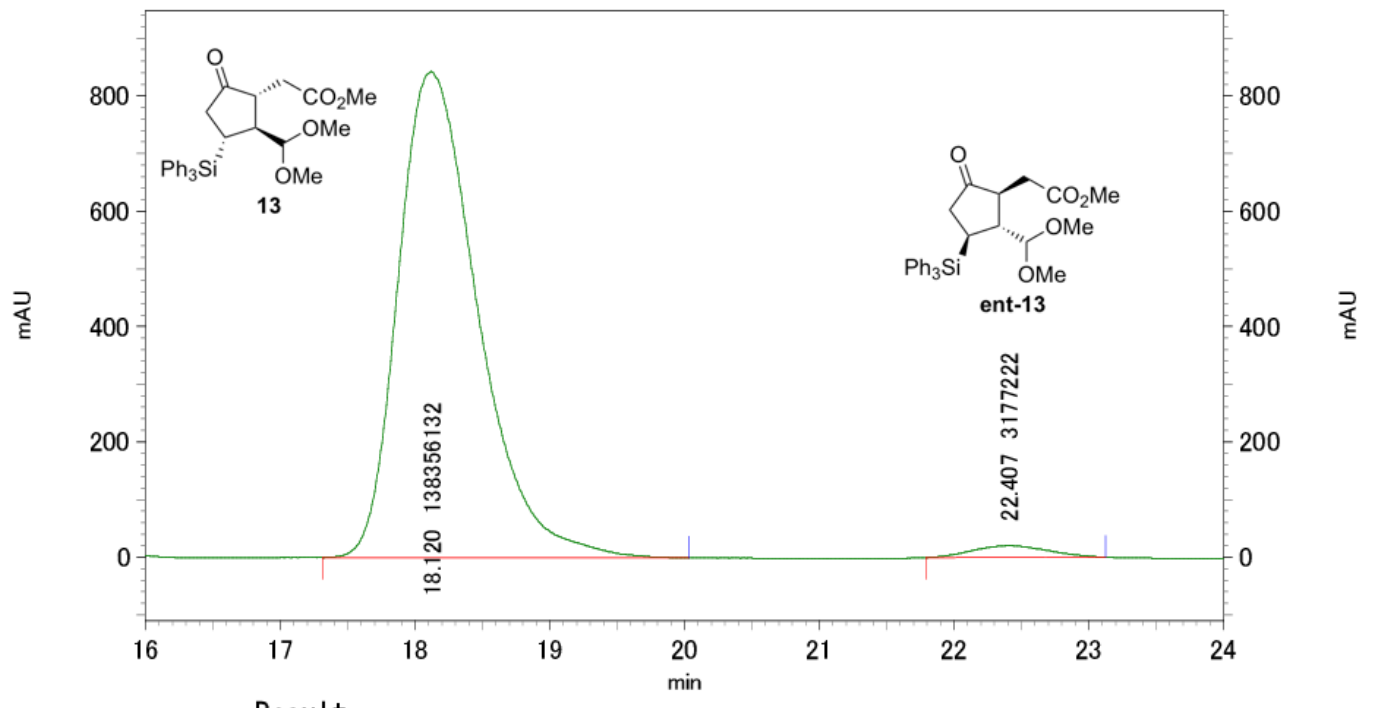

1: $214 \mathrm{~nm}, 4$ nm結果

Pk \# Retention time / min

Integration/\%

2. 245

\begin{tabular}{|r|r|r|}
\hline$r-$ 多 & 100.000 \\
\hline
\end{tabular} 


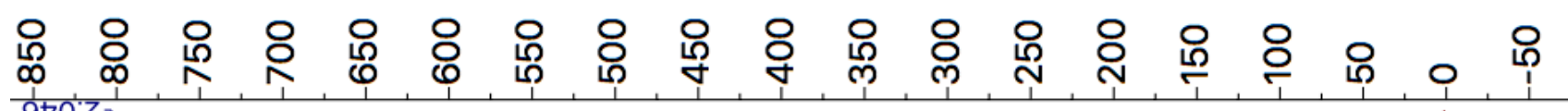

$9700^{\prime} z$

$\varepsilon 90$ '

090 ' 2

$6 \angle 0^{\circ} Z$

980 ' 2

$160 ' z$

660 ' 2

SOl'z-

$\varepsilon l l ' z$

ISG'Z -

$999^{\prime} Z$

$\angle L Q^{\prime} Z$.

$\varepsilon 89^{\prime} Z$

$169^{\prime} Z$

969 ' $Z$

LI9 Z

Z๑9'

$899^{\circ} Z$

$\nabla \angle L Z Z$

$26 L^{2} Z-$

$966 \cdot 2$

$690^{\circ} \varepsilon-$

ozz $\varepsilon$

$\nabla \angle 9^{\circ} \varepsilon$

$189^{\circ} \varepsilon^{\gamma}$

$789^{\circ} 9$

989'

$689^{\circ} 9$

Z69' $9-1$

969.

$\angle 699^{\circ}$

$99 \varepsilon<1$

$09 \varepsilon^{\circ} L$

$\varepsilon 9 \varepsilon^{\circ} L$

$89 \varepsilon \angle$

ZLE' $\angle$

$\left.\angle L \varepsilon^{\prime} \angle\right]$

$08 \varepsilon^{\circ} L$

$98 \varepsilon^{\circ} \angle$

Z6E' $L$

G6E $L$

ZLt $L$

$9 L t \angle$

OZt $\angle$

$\angle Z \nabla \angle$

$\nabla \varepsilon \nabla \cdot L$

$\triangle 99^{\circ} \angle$

$899^{\circ} \angle$ -

$089^{\circ} L$

$789^{\circ} \angle$

$889^{\circ} L$
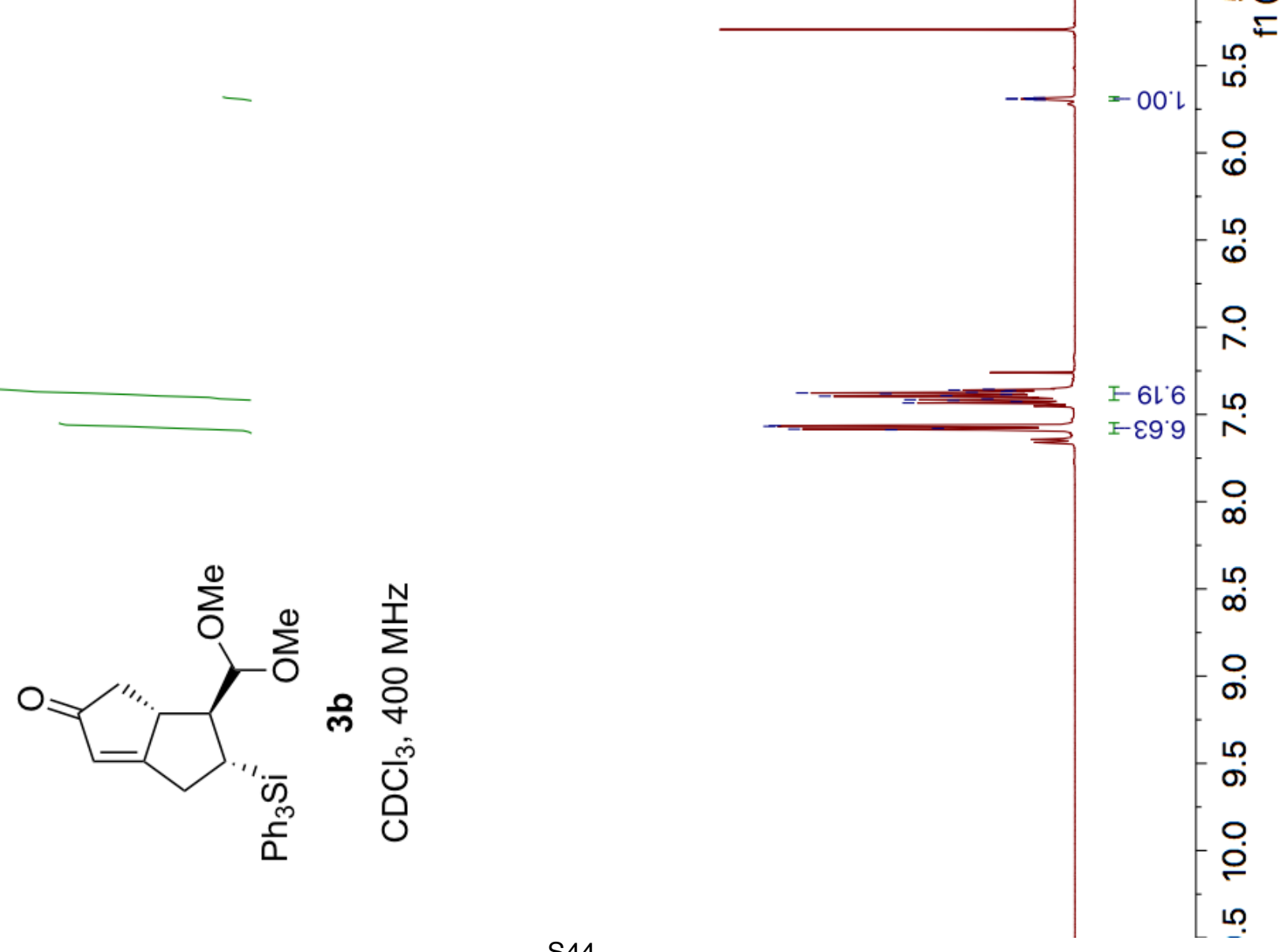
兽 哭

งเง๋๖Z-

$\angle 9 \mathcal{C}^{\circ} 0 \varepsilon^{-}$

ฤ6乙'ยฤ

89.'97-

$899^{\circ} 67-$

LZL'99)

98t'99

$189.9 /$

$000 \angle L T$

$81 \varepsilon^{\prime} L L$

\&6ฑ 90L-

$\nabla 66^{\circ} \nabla$ 乙L

\&†0.8ZL]

$0 \angle 08 \mathrm{ZL}$

แะ8 6L-

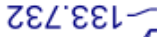

9ع8 $9 \varepsilon 1-1$

976. $9 \varepsilon \mathrm{L}$

$\angle Z L 68 L-$

ย9จ・レてー
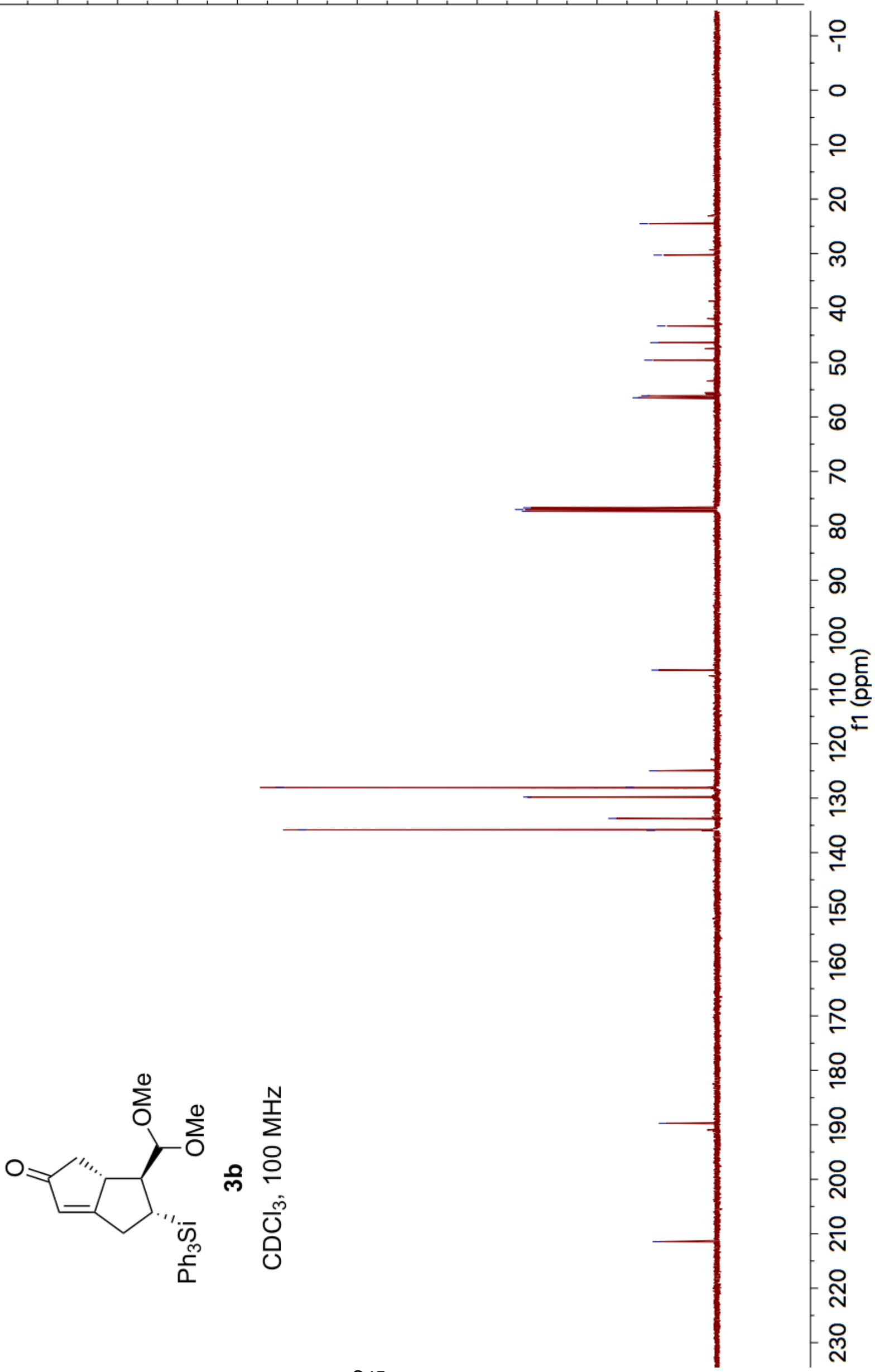


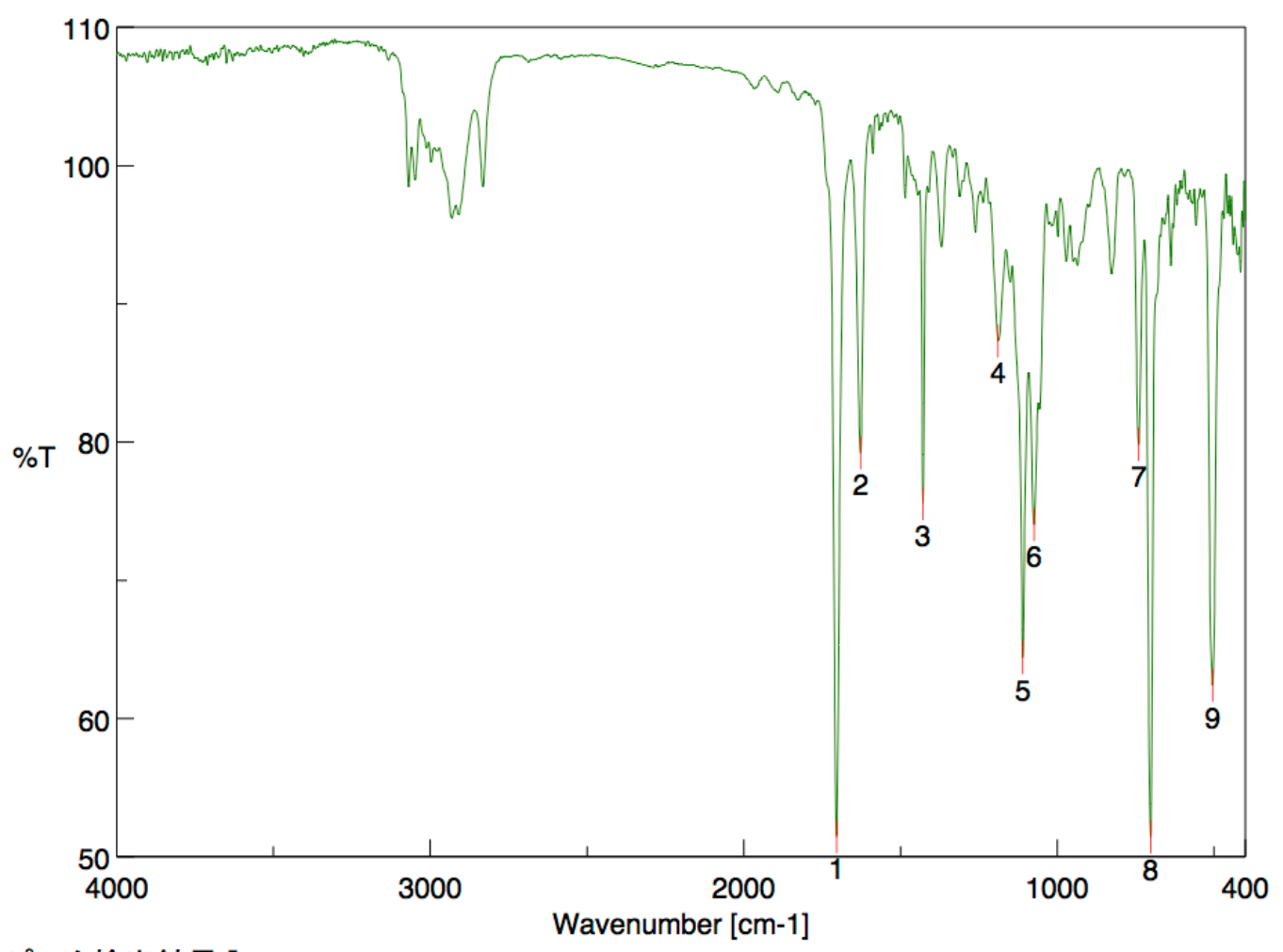

[ピーク検出結果 ]

$\begin{array}{llllll}\text { No. } & \text { 位置 } & \text { 強度 } & \text { No. } & \text { 位置 } & \text { 強度 } \\ 1 & 1703.8 & 51.4492 & 2 & 1627.63 & 79.2141 \\ 3 & 1428.03 & 75.544 & 4 & 1188.9 & 87.2977 \\ 5 & 1109.83 & 64.4062 & 6 & 1074.16 & 74.0161 \\ 7 & 740.531 & 79.802 & 8 & 701.962 & 51.4224 \\ 9 & 504.294 & 62.4195 & & & \end{array}$<smiles>COC(OC)[C@@H]1[C@H]([SiH2]c2ccccc2)CC2=CC(=O)C[C@@H]21</smiles> 


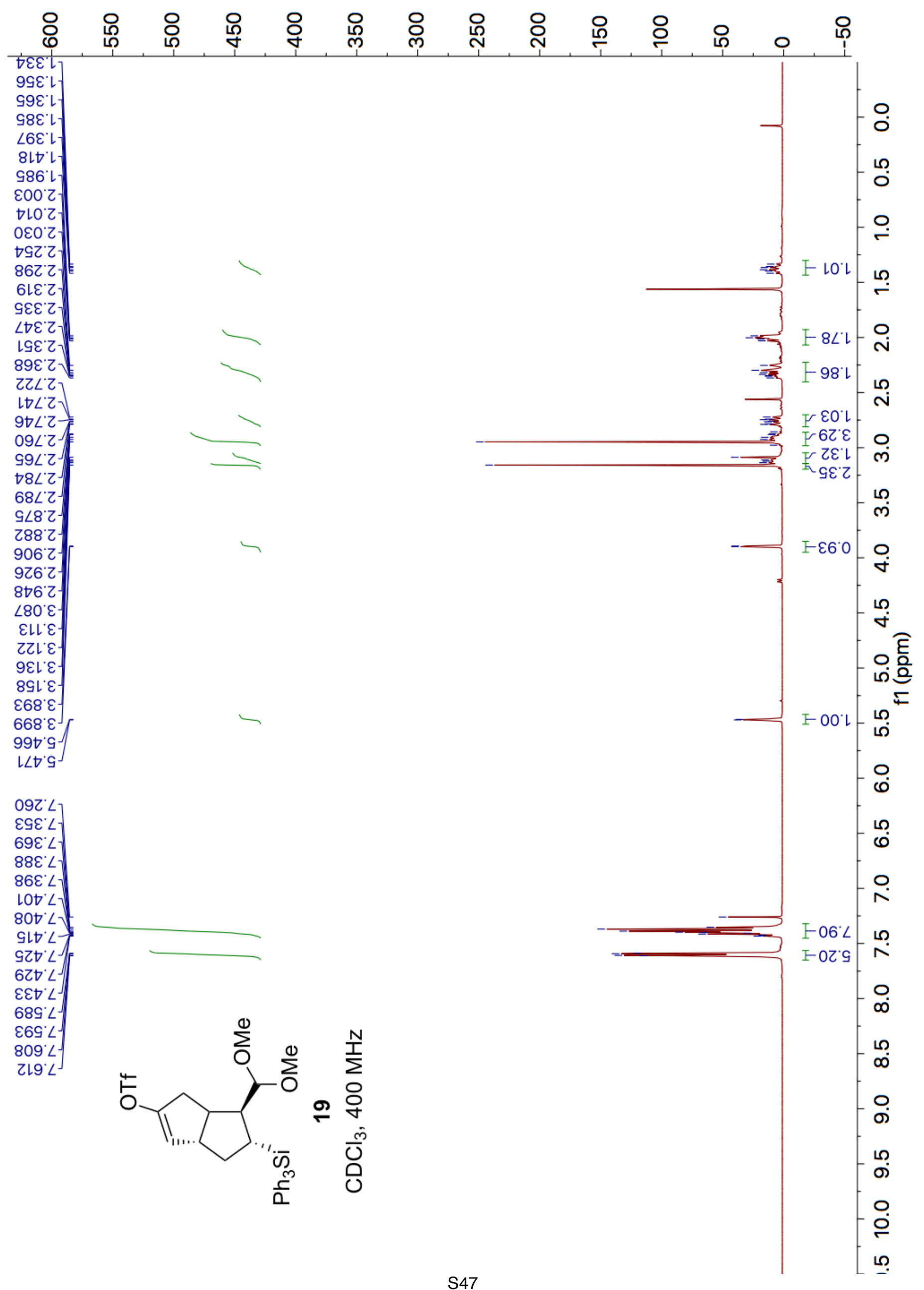




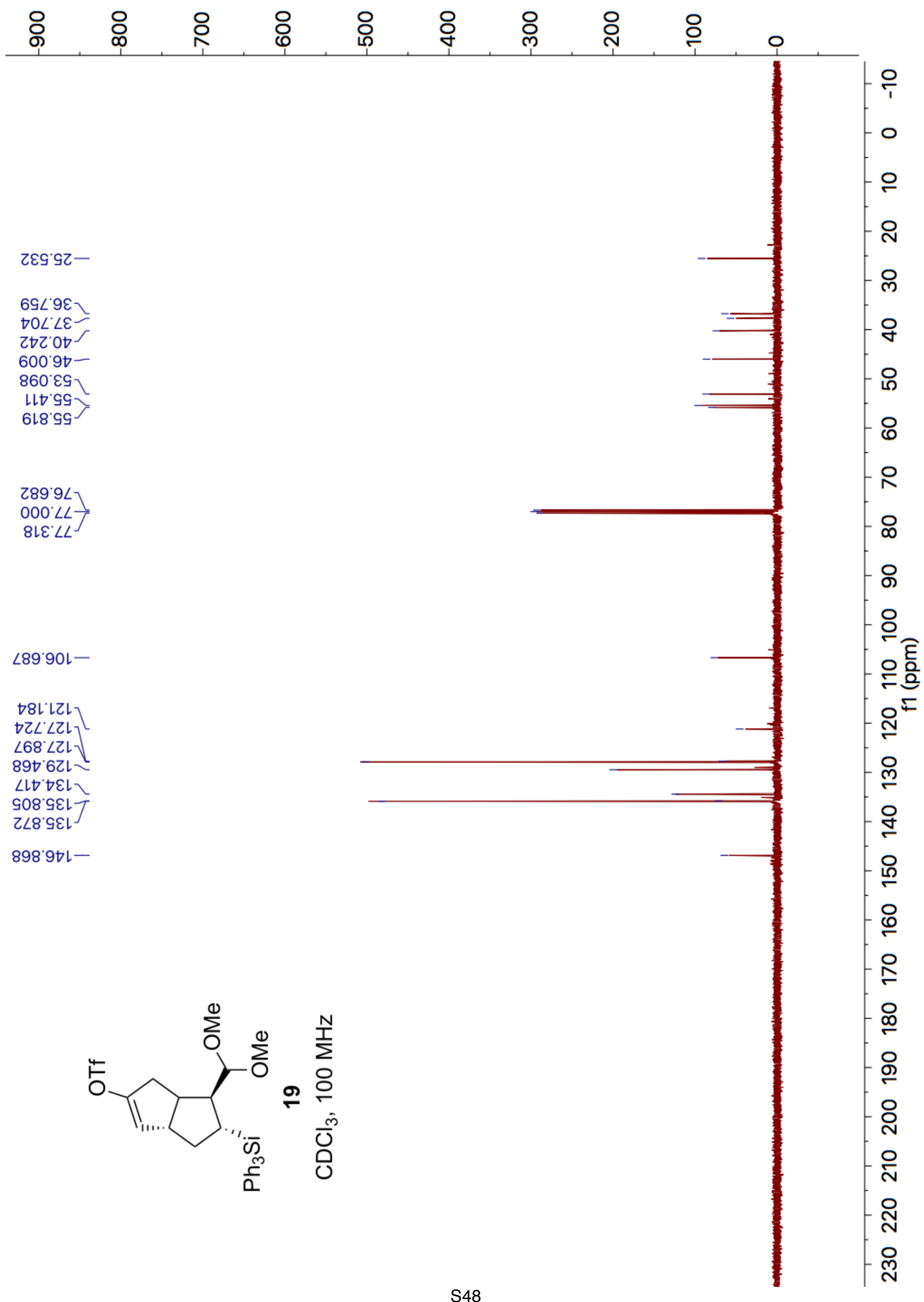




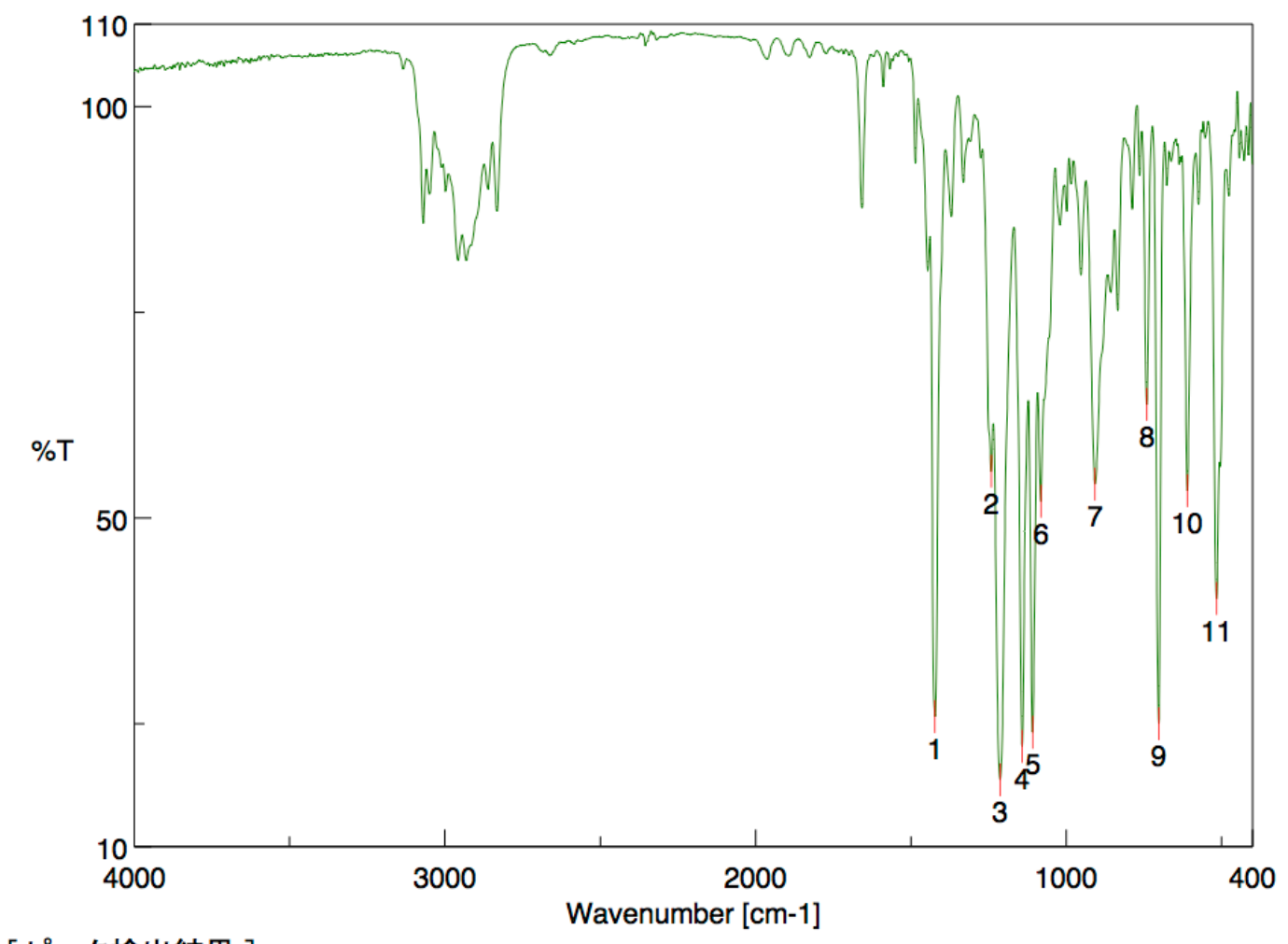

[ピーク検出結果 ]

$\begin{array}{llllll}\text { No. } & \text { 位置 } & \text { 強度 } & \text { No. } & \text { 位置 } & \text { 強度 } \\ 1 & 1422.24 & 25.8027 & 2 & 1240.97 & 55.6413 \\ 3 & 1213.01 & 18.1352 & 4 & 1141.65 & 22.1752 \\ 5 & 1107.9 & 23.9145 & 6 & 1081.87 & 51.9883 \\ 7 & 907.344 & 54.0807 & 8 & 740.531 & 63.7831 \\ 9 & 701.962 & 24.9407 & 10 & 610.36 & 53.2941\end{array}$<smiles>CCCC1=C[C@@H]2C[C@H]([SiH2]c3ccccc3)[C@H](C(OC)OC)C2C1</smiles>

19 
呬

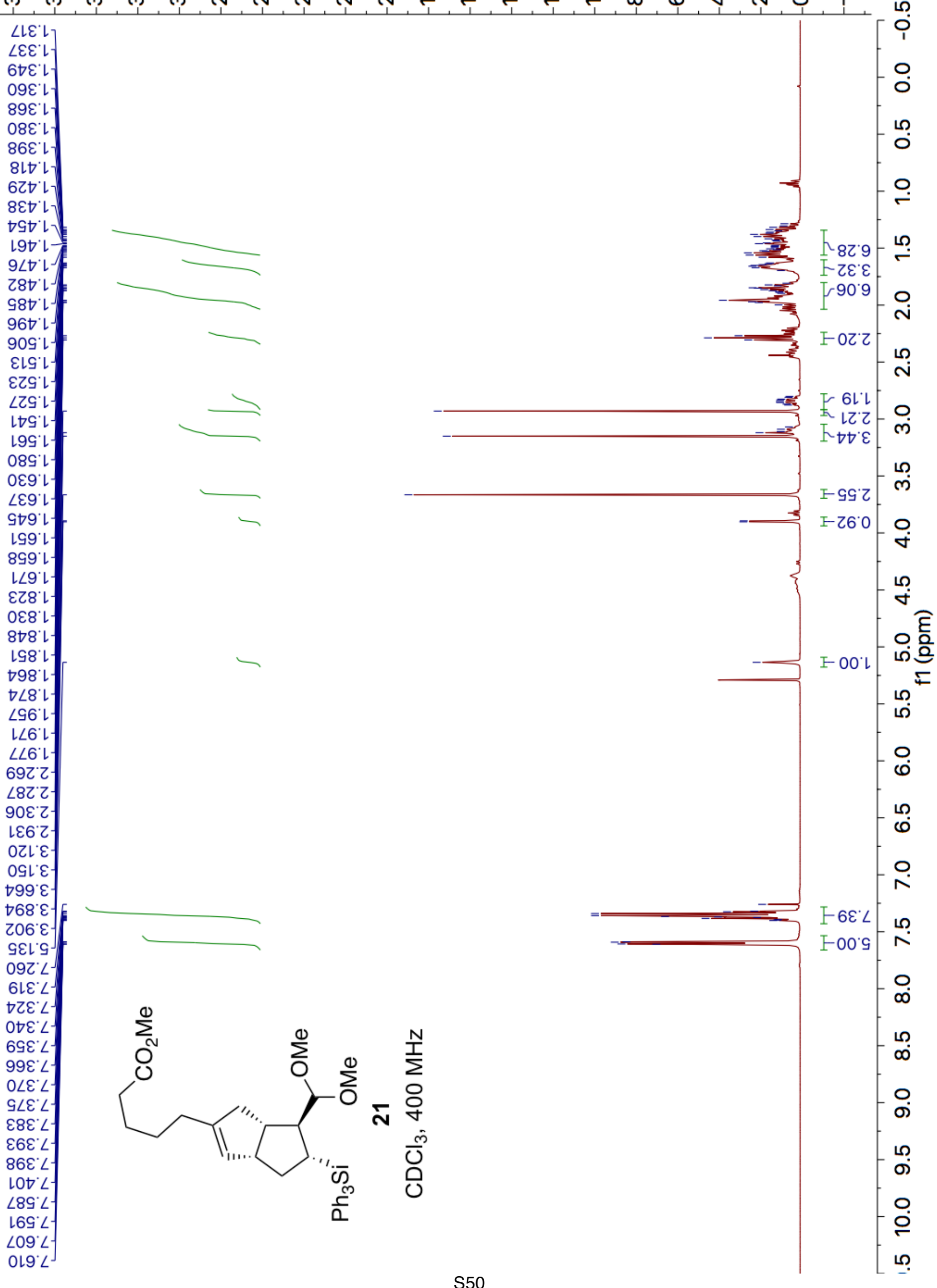




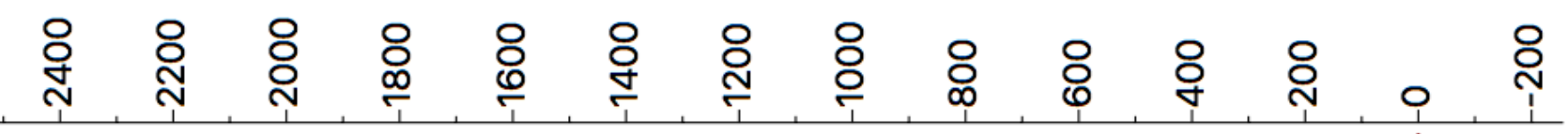

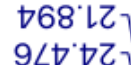

tul. 92

$890^{\circ} \angle Z$

$8 \varepsilon \nabla^{\circ} 0 \varepsilon$

076.

978 ' $\varepsilon \varepsilon^{\prime}$

$99 Z^{\circ} \angle \varepsilon^{J}$

$889^{\prime} L t^{\prime}$

09t $\varepsilon t^{\circ}$

LVT LG-

LZヤ SG-

$\nabla \nabla 8^{\circ} 0<1$

9l9.9L

घย6. $9 L$

ZGน $\angle L$

L $\angle \mathcal{G}^{\circ} \angle \mathrm{OL}-$

$989^{\circ} \angle Z L$

980.6ZL-

OLL'GEL

๑๐ $9 \varepsilon L-$

ZZ6.9EL

เE9เเ७レ

$\varepsilon 60^{\circ} \triangleright \angle L-$

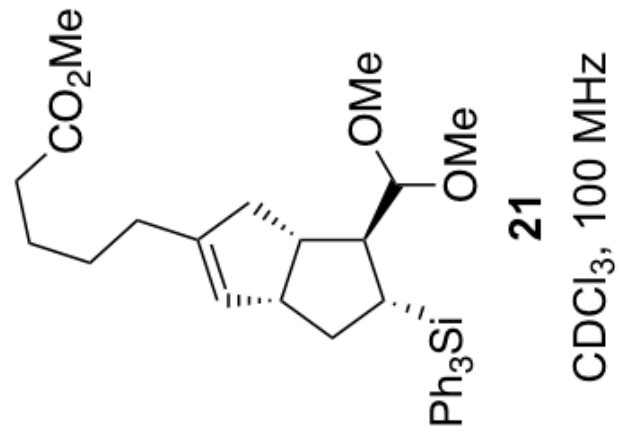

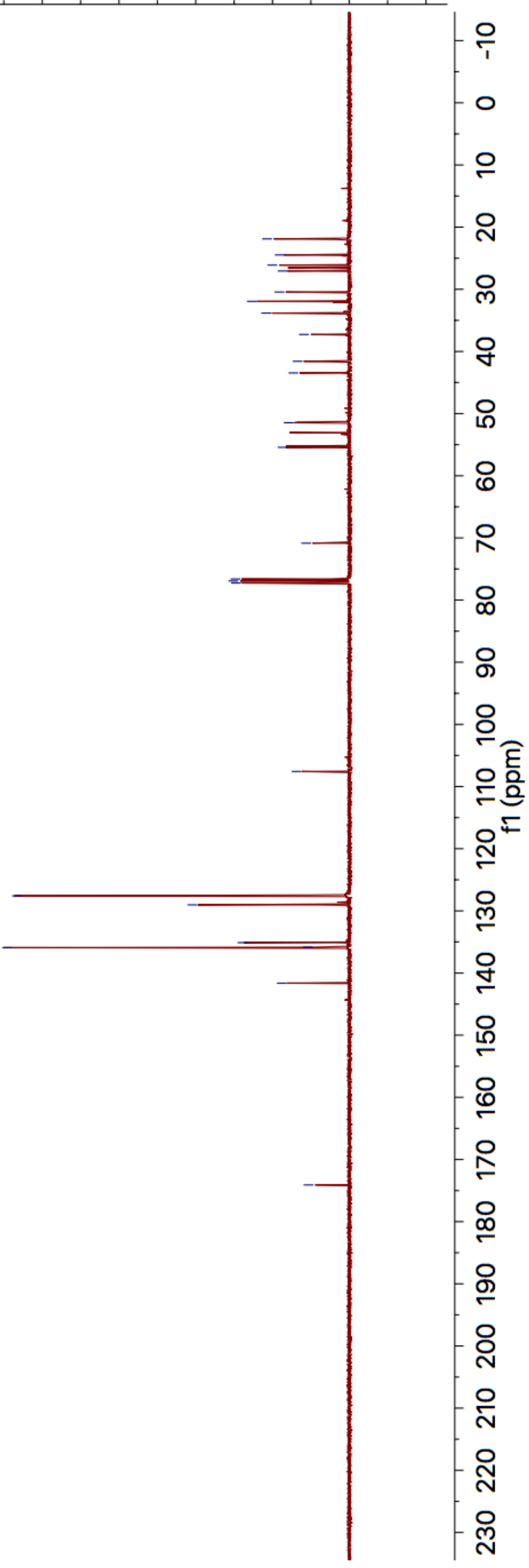




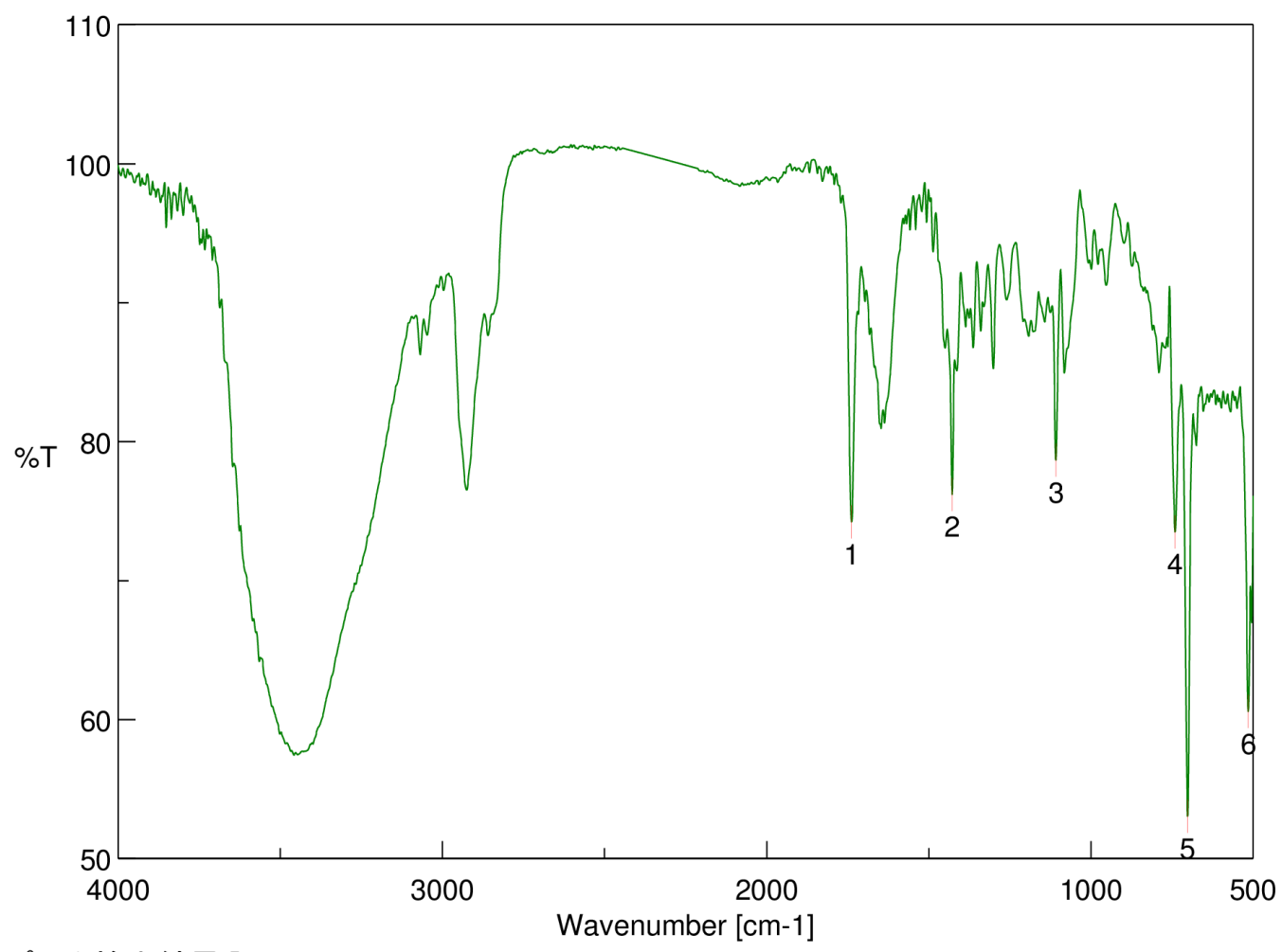

[ピーク検出結果 ]

$\begin{array}{llllll}\text { No. } & \text { 位置 } & \text { 強度 } & \text { No. } & \text { 位置 } & \text { 強度 } \\ 1 & 1738.51 & 74.2216 & 2 & 1428.03 & 76.2043 \\ 3 & 1107.9 & 78.6544 & 4 & 740.531 & 73.508 \\ 5 & 701.962 & 53.0568 & 6 & 514.901 & 60.5768\end{array}$

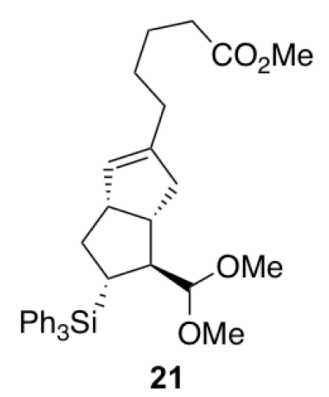




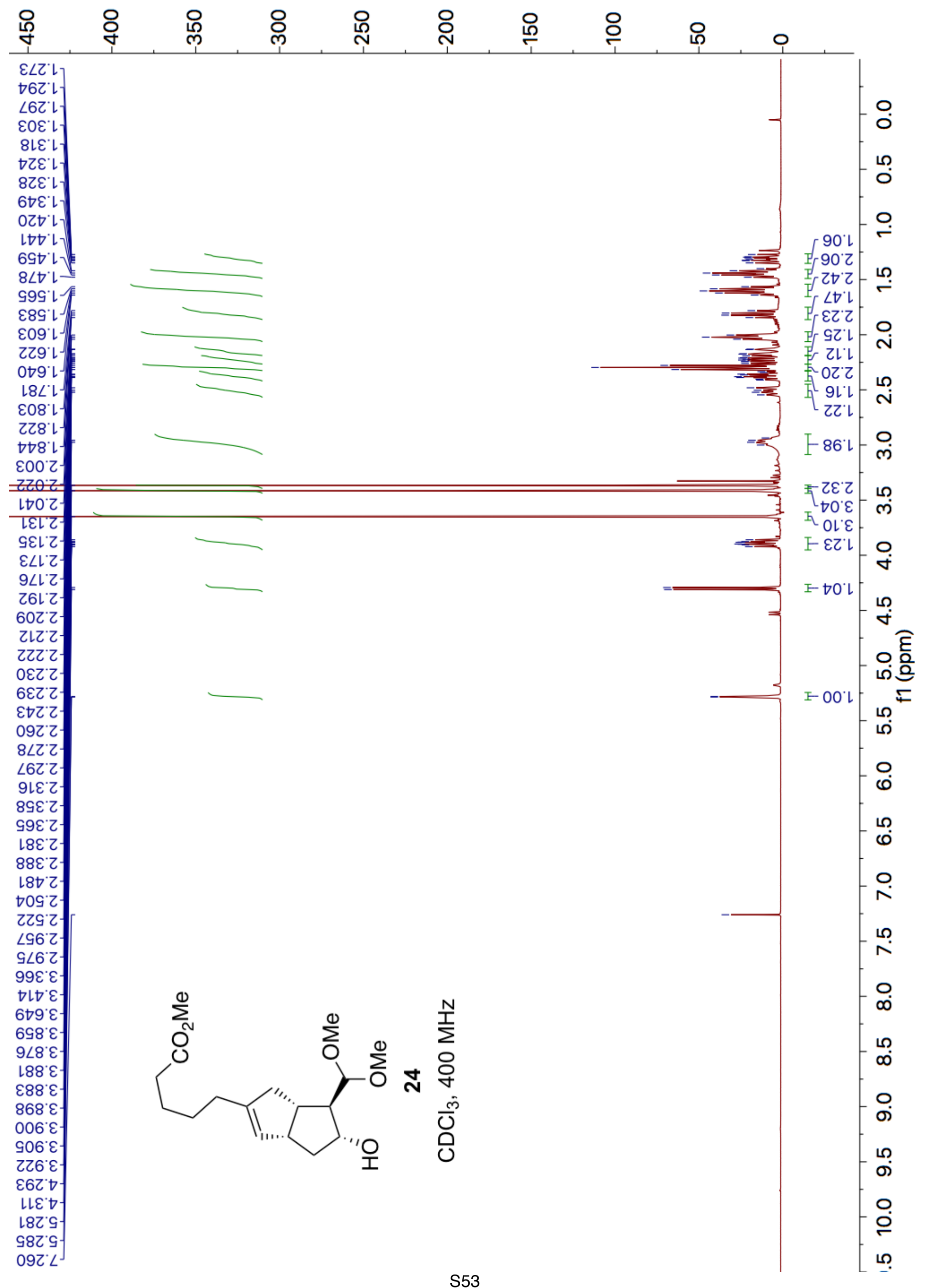




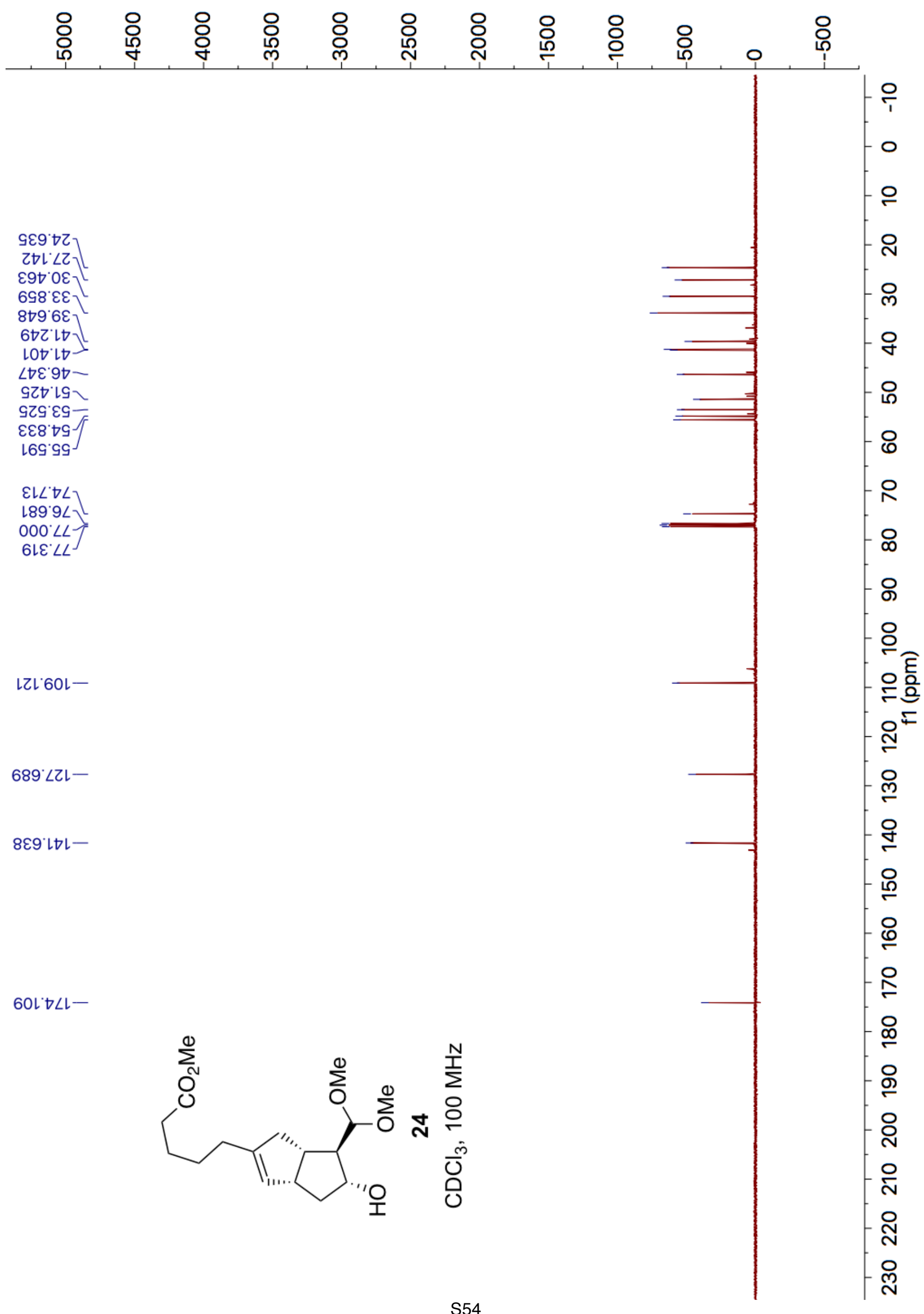




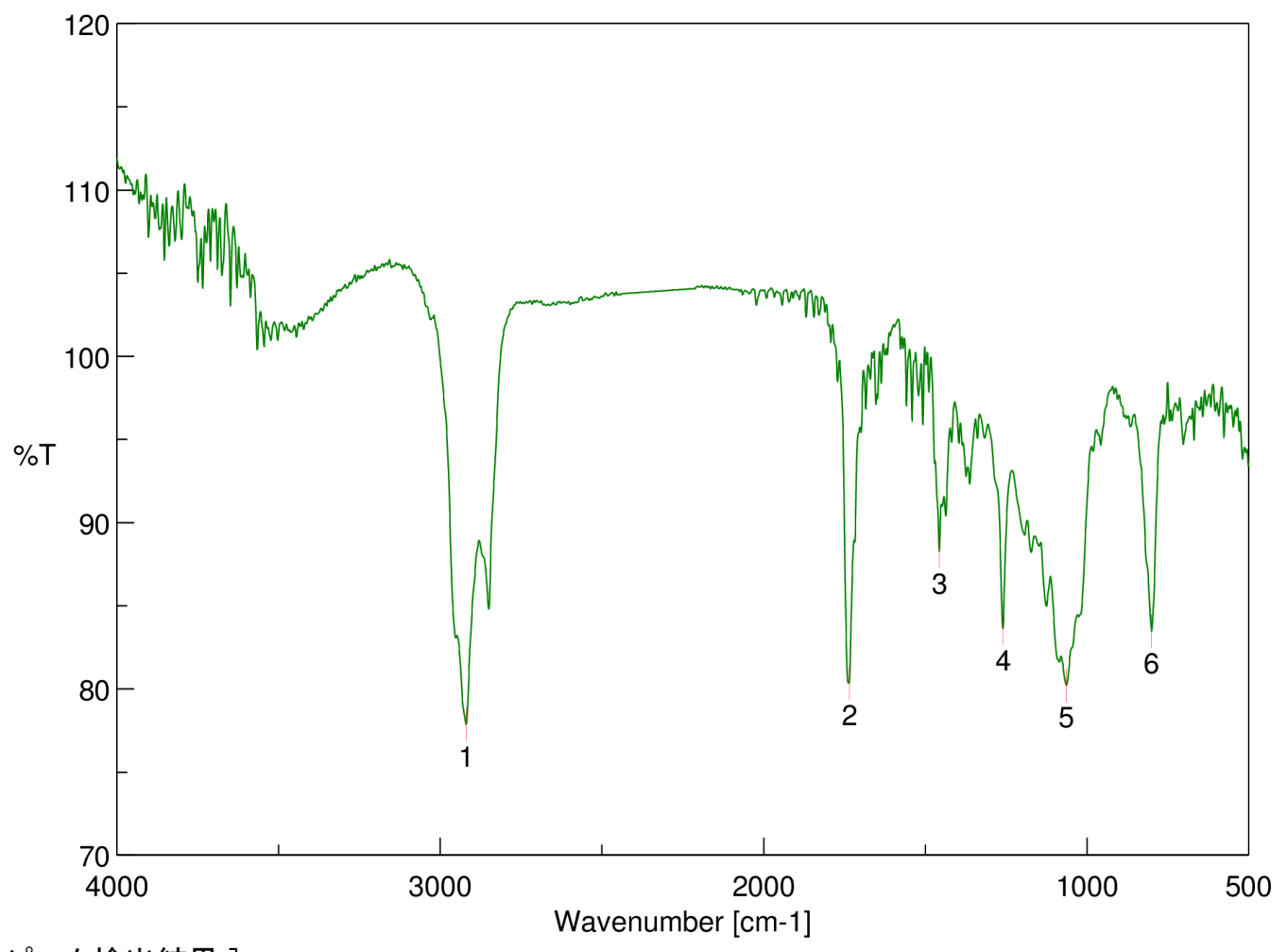

[ピーク検出結果 ]

$\begin{array}{llllll}\text { No. } & \text { 位置 } & \text { 強度 } & \text { No. } & \text { 位置 } & \text { 強度 } \\ 1 & 2919.7 & 77.8559 & 2 & 1735.62 & 80.3509 \\ 3 & 1456.96 & 88.2767 & 4 & 1260.25 & 83.6221 \\ 5 & 1063.55 & 80.2215 & 6 & 800.314 & 83.4685\end{array}$

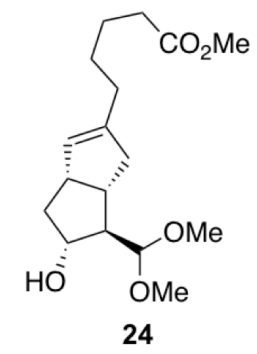




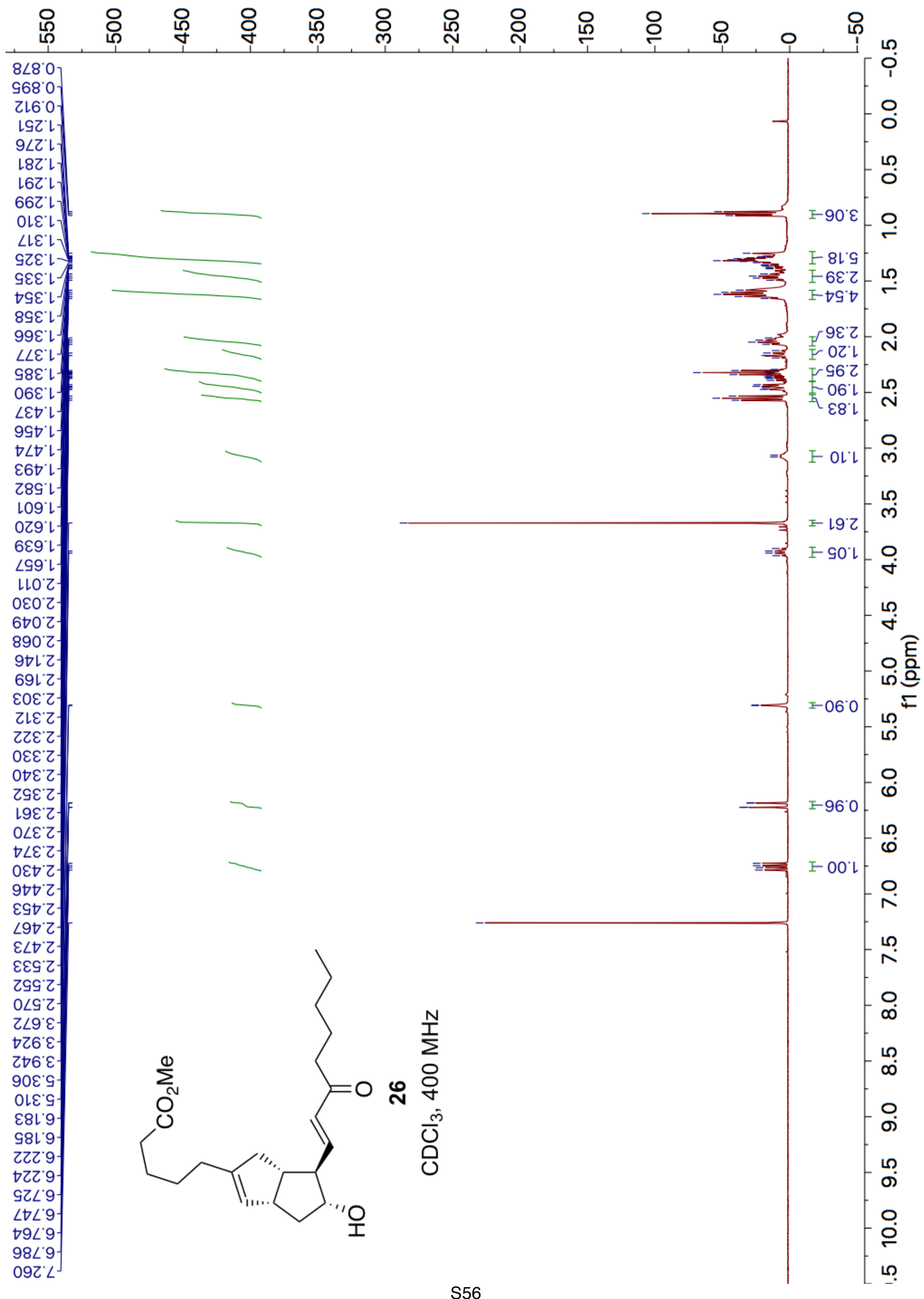




\section{惫离员}

$\angle \triangleright 6 \cdot \varepsilon L-$

9८७乙乙

908' $\varepsilon 2 \beth$

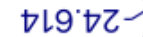

$60 L^{\circ} \angle Z^{J}$

68t $0 \varepsilon^{\circ}$

$G \angle \nabla^{\circ}\llcorner\varepsilon$

เG8' $\varepsilon \varepsilon^{\prime}$

งะ8 $6 \varepsilon$

$8 \mathrm{~L} 0 \mathrm{t}^{-1}$

GEG 07

เ9Z" $\nabla t$

866 ' $9 t$

SLG'L

890.89

Z89.9L

$000^{\circ} \angle L=$

$80 Z^{\prime} \angle L$

$6 L \varepsilon^{\prime} \angle L$
0ZL'8ZL-

เOL'LEL-

งเง๋เレレ-

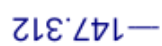

$\left\llcorner\varepsilon L^{\circ} \nabla \angle L-\right.$

GEG'00Z-

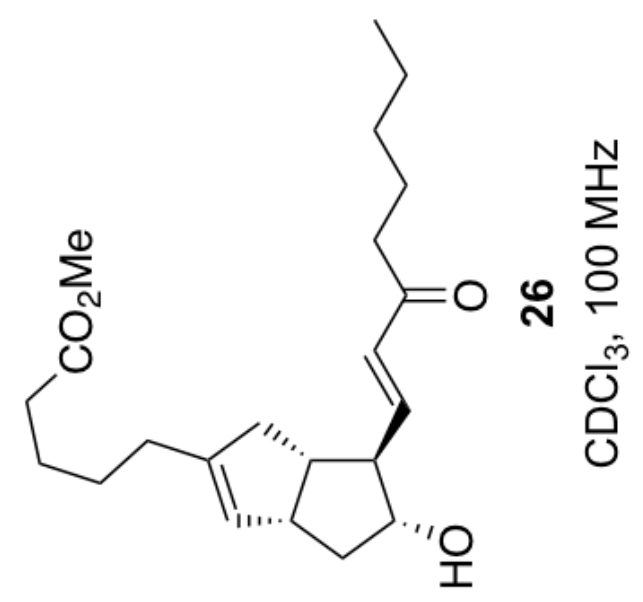




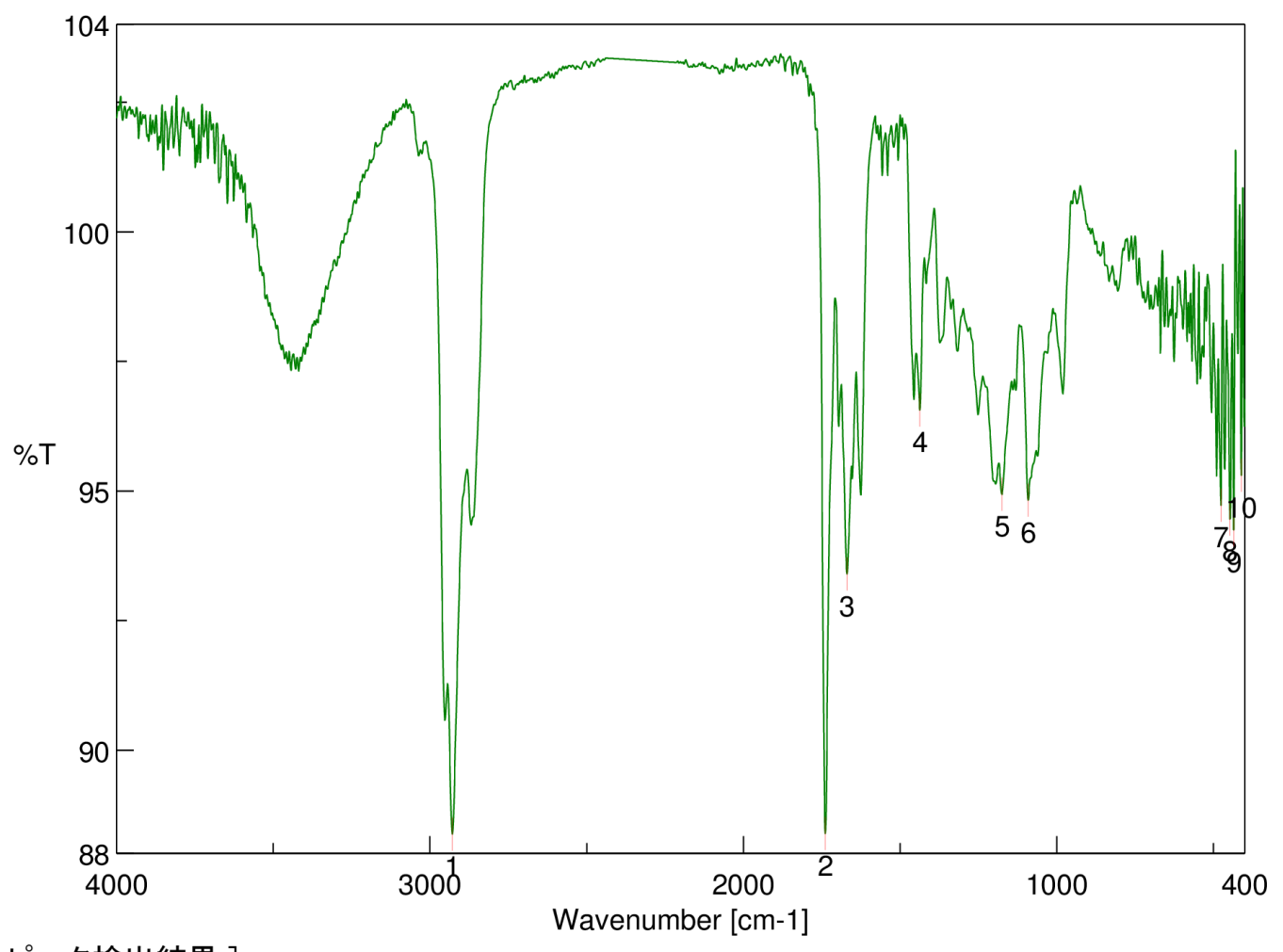

[ピーク検出結果 ]

$\begin{array}{llllll}\text { No. } & \text { 位置 } & \text { 強度 } & \text { No. } & \text { 位置 } & \text { 強度 } \\ 1 & 2928.38 & 88.3711 & 2 & 1738.51 & 88.3893 \\ 3 & 1669.09 & 93.391 & 4 & 1436.71 & 96.5538 \\ 5 & 1175.4 & 94.9283 & 6 & 1091.51 & 94.8153 \\ 7 & 476.331 & 94.7161 & 8 & 447.404 & 94.4537 \\ 9 & 434.869 & 94.2464 & 10 & 410.763 & 95.3007\end{array}$

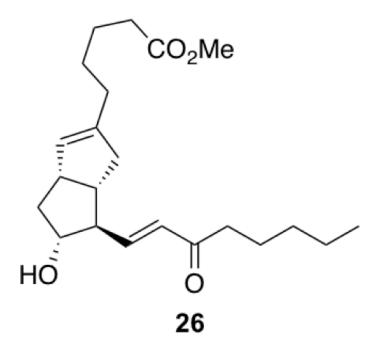




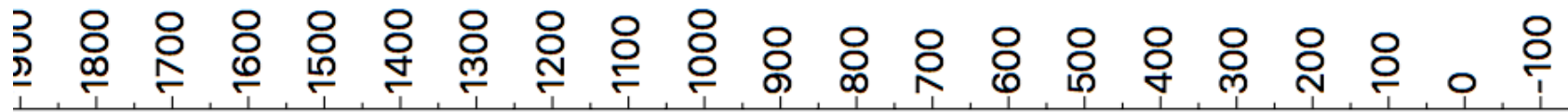

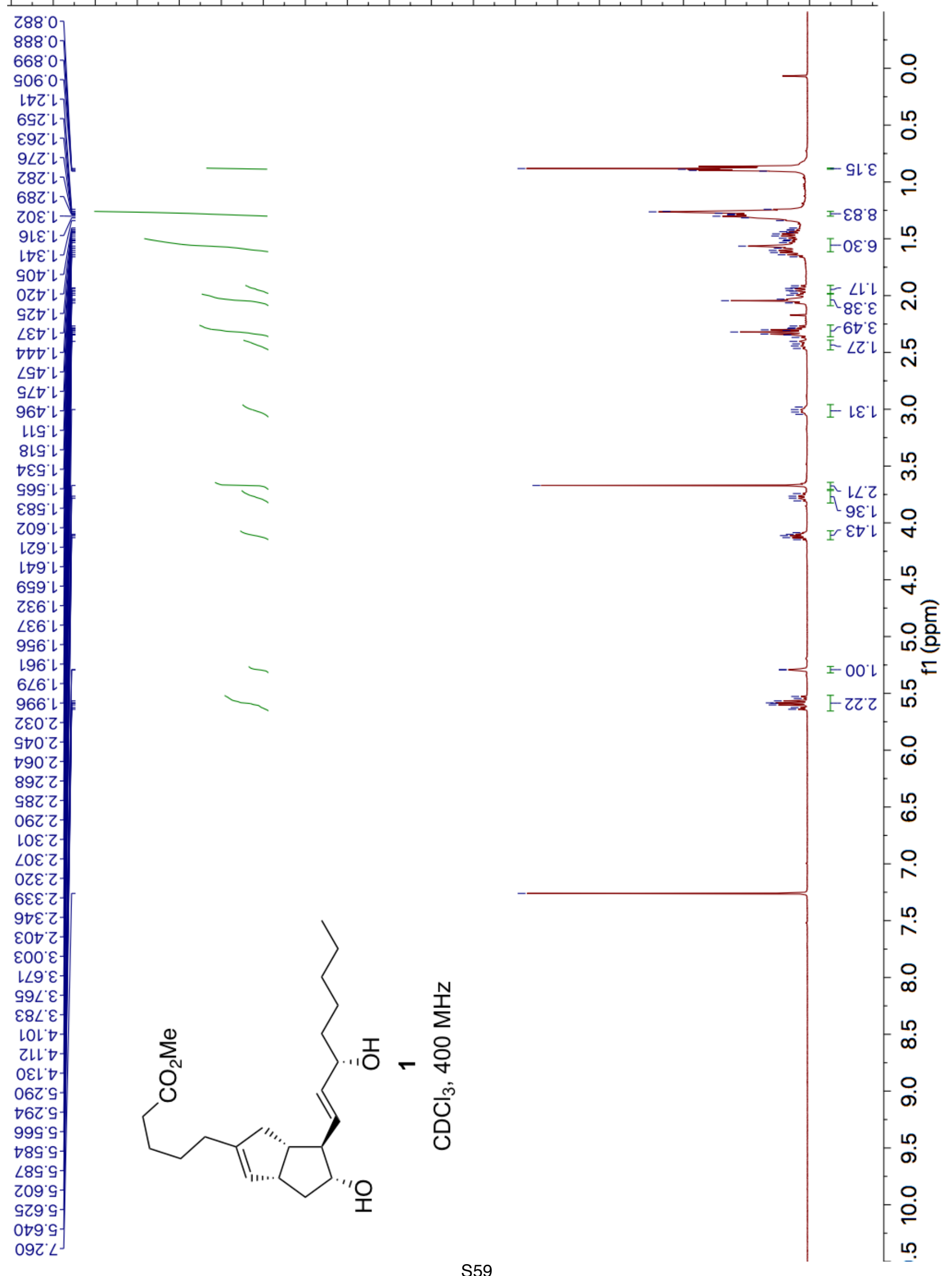




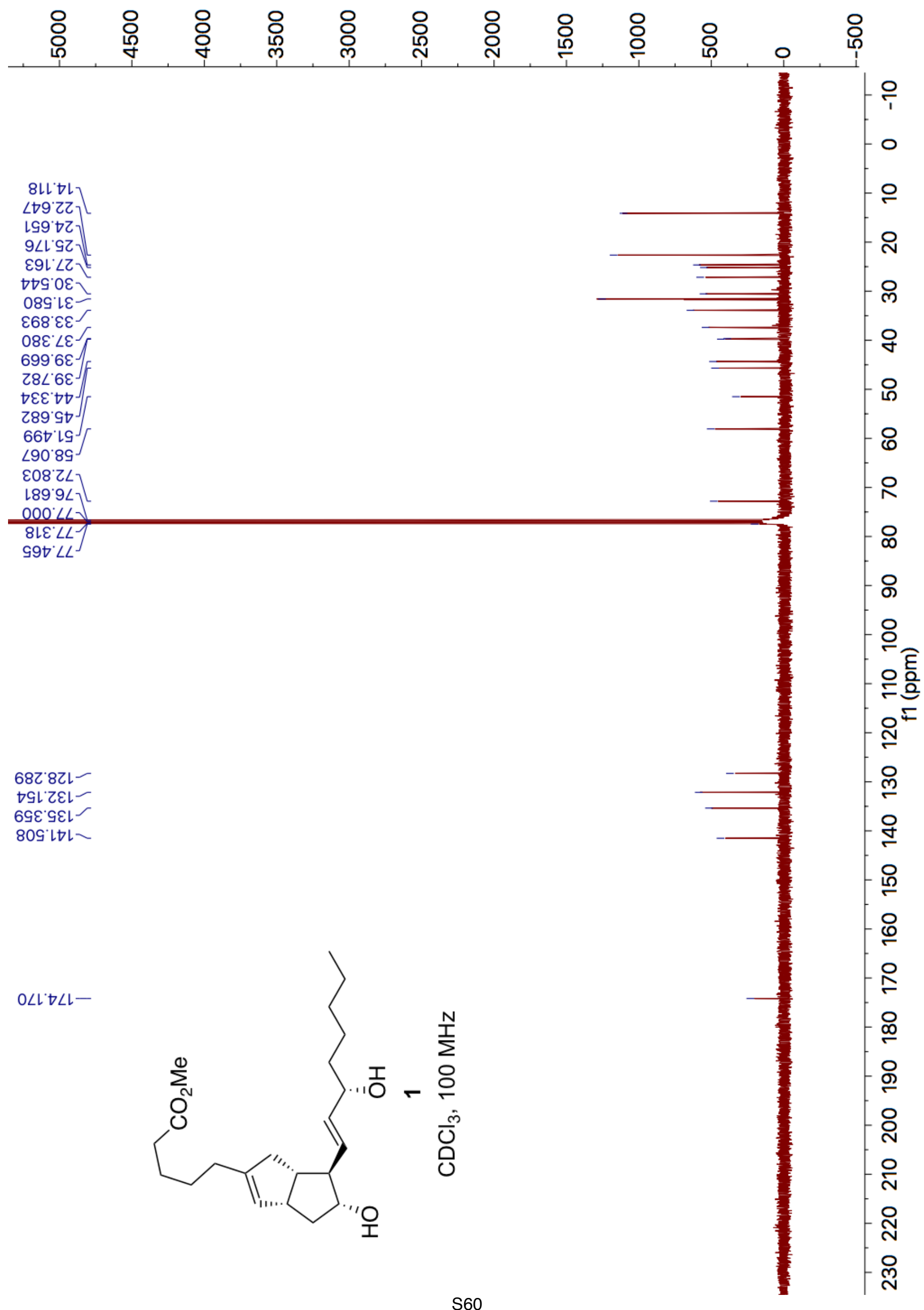




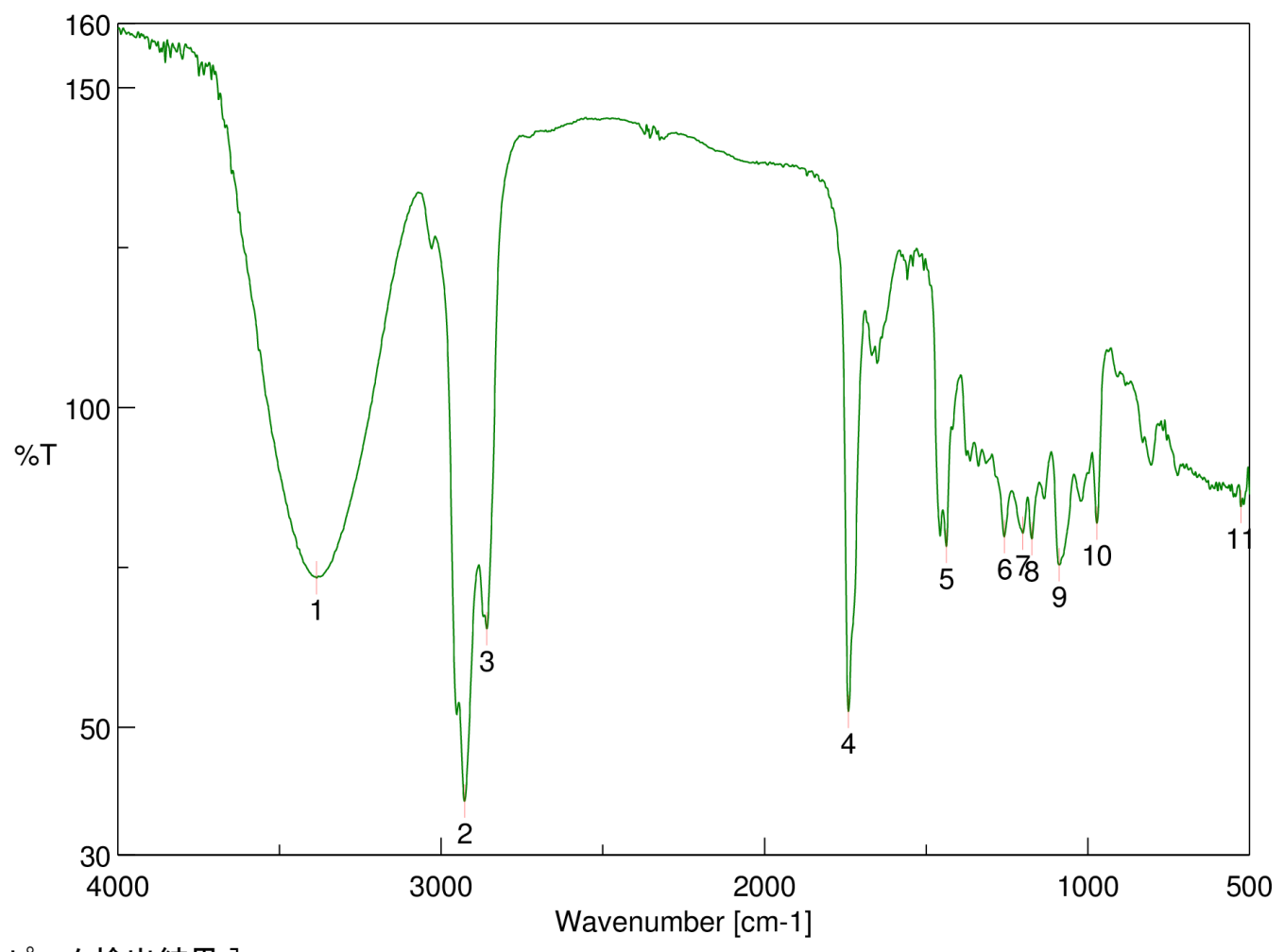

[ピーク検出結果 ]

$\begin{array}{llllll}\text { No. } & \text { 位置 } & \text { 強度 } & \text { No. } & \text { 位置 } & \text { 強度 } \\ 1 & 3385.42 & 73.3409 & 2 & 2927.41 & 38.4237 \\ 3 & 2858.95 & 65.3821 & 4 & 1740.44 & 52.4467 \\ 5 & 1437.67 & 78.2714 & 6 & 1258.32 & 79.7468 \\ 7 & 1201.43 & 80.3372 & 8 & 1173.47 & 79.4508 \\ 9 & 1088.62 & 75.3635 & 10 & 971.947 & 81.8756 \\ 11 & 526.471 & 84.462 & & & \end{array}$

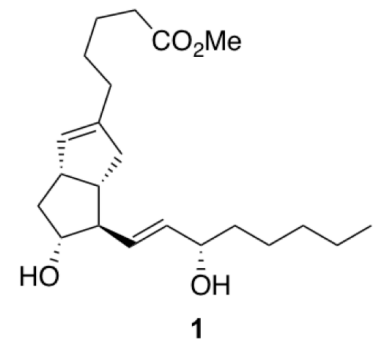




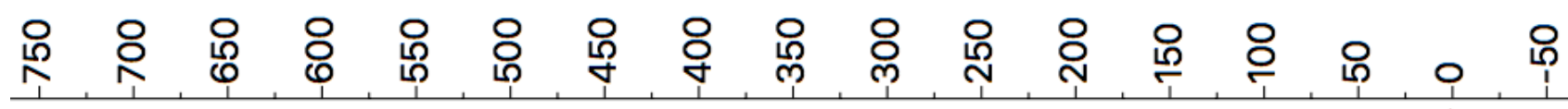

овะ 0

$88 \varepsilon^{\circ} 0$

$6 \angle D^{\circ}$

OLG'

EES'
Z9'.

$\nabla t l^{\prime} Z$

$\angle \angle L ' Z$

เ6L'z

$\varepsilon 乙 Z$ Z

Zง\&'て-

$69 \varepsilon^{\prime} 乙$

乙৪६'て

OLt'

GEt'Z

SSG'Z]

$\angle 99^{\circ} Z$ ]

$189^{\circ} Z$

Z69' 2

$9+9{ }^{\circ} z$

ง99' $\mathrm{C}$

$999^{\circ} \mathrm{Z}$

$889 \cdot \mathrm{Z}$

$869 ' z$

$60 \angle 27$

$8 L L Z$.

$6 Z \angle ' Z$ -

$0 t \angle ' Z$ -

$z 9 \angle 2$

$Z \angle L ' Z$

$\nabla 8 \angle ' Z$

$69 l^{\prime} \varepsilon$

$\varepsilon 69^{\prime} \varepsilon$

$606^{\circ} \varepsilon$

$\angle 16{ }^{\circ} \varepsilon$

$092 \angle$

L $\angle E L L$

8Z9' $L$
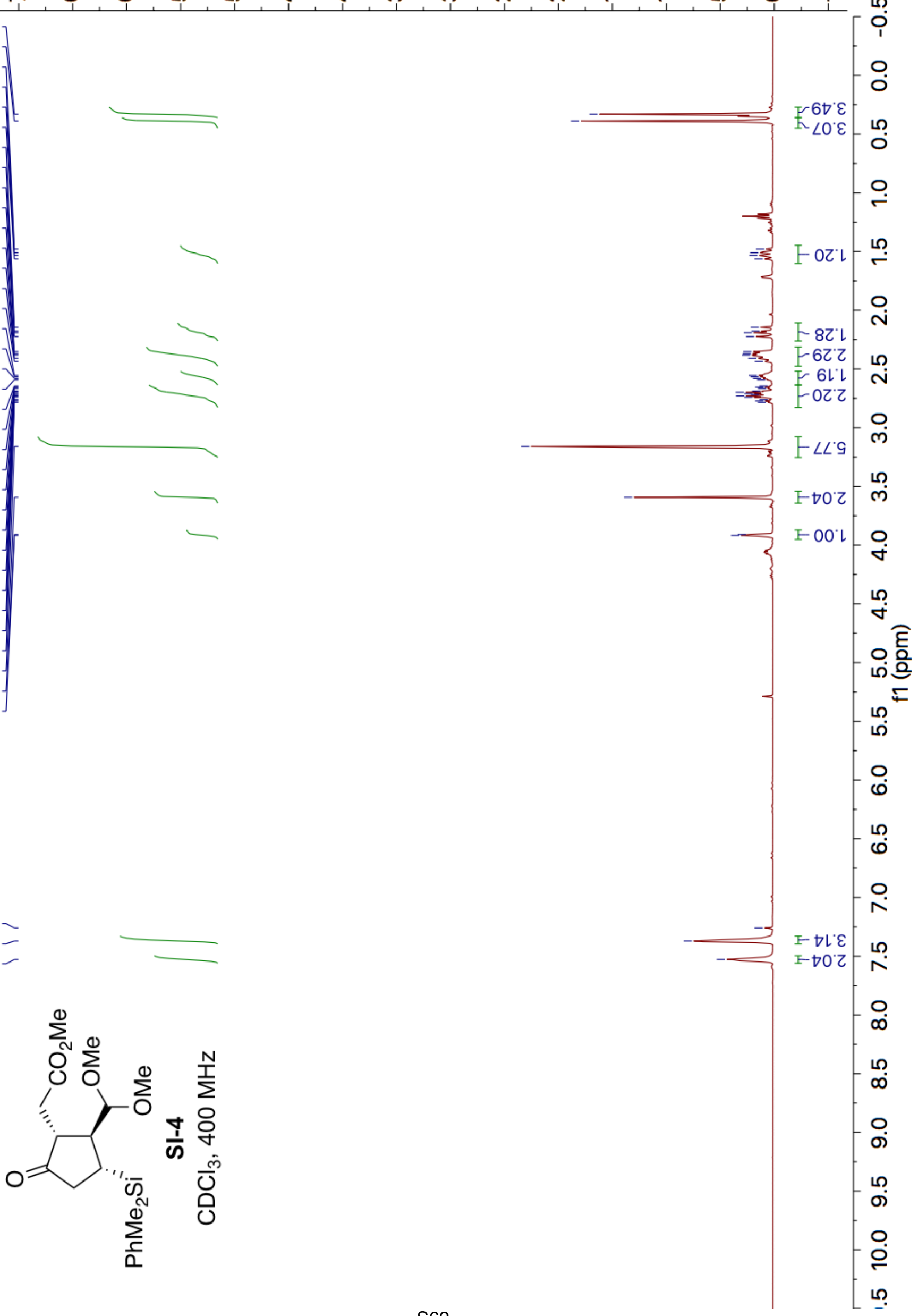
整 尔

GE乙' $\mathrm{G}^{-} ح$

$0<\nabla^{\circ} \varepsilon-$

ヤ乙Lレてー

IG9 ${ }^{\circ} \varepsilon$ -

l00.07

809.97

6จオ'L

$6 \angle 0.99$

น०ع'99-

$189^{\circ} 9 /$

$000^{\circ} \angle L \frac{1}{T}$

$6 L \varepsilon^{\prime} L L$

OZE $\angle O L-$

$6 Z 6^{\circ} \mathrm{LZL}=$

89Z6ZL

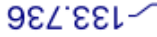

000'8हL -

เ69'ZLL-

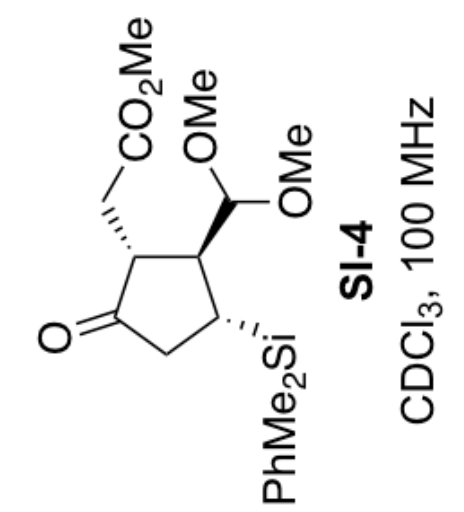

$000612-$

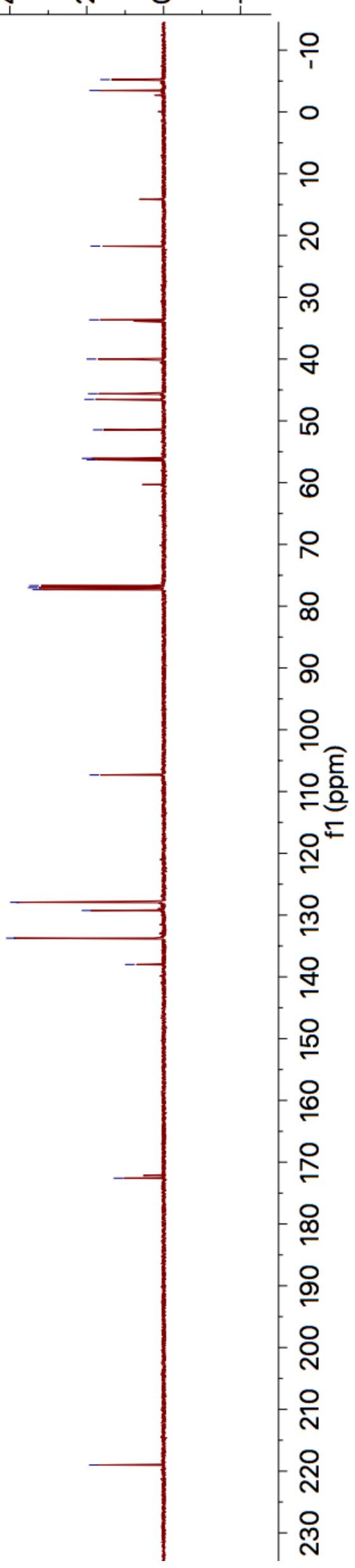




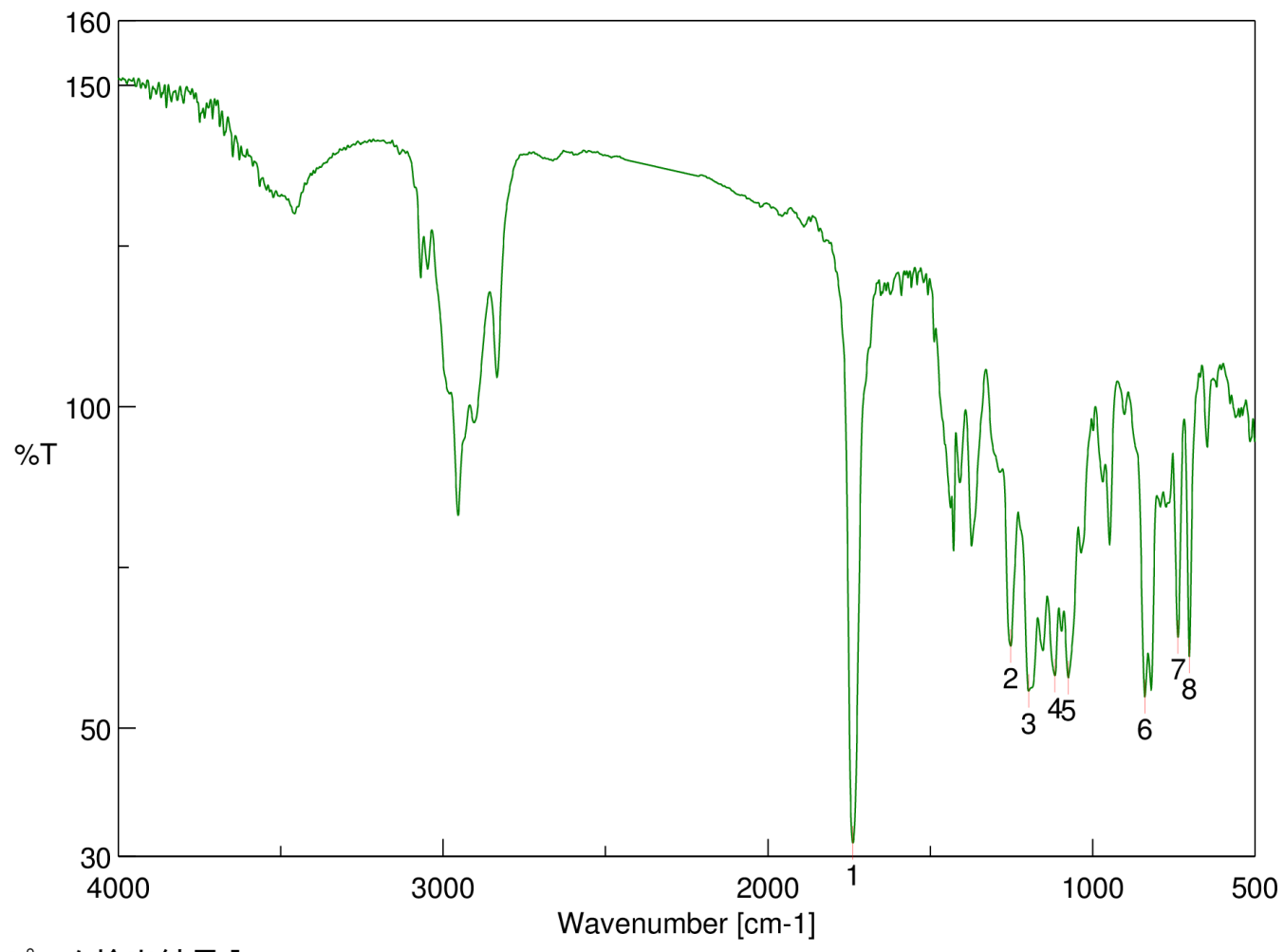

[ピーク検出結果 ]

$\begin{array}{llllll}\text { No. } & \text { 位置 } & \text { 強度 } & \text { No. } & \text { 位置 } & \text { 強度 } \\ 1 & 1738.51 & 32.0972 & 2 & 1252.54 & 62.7078 \\ 3 & 1196.61 & 55.7295 & 4 & 1116.58 & 58.1474 \\ 5 & 1075.12 & 57.7988 & 6 & 838.883 & 54.8568 \\ 7 & 737.639 & 64.1552 & 8 & 701.962 & 61.0943\end{array}$<smiles>COC(OC)[C@H]1[C@@H](CC(C)=O)CC(=O)[C@H]1[SiH3]</smiles> 


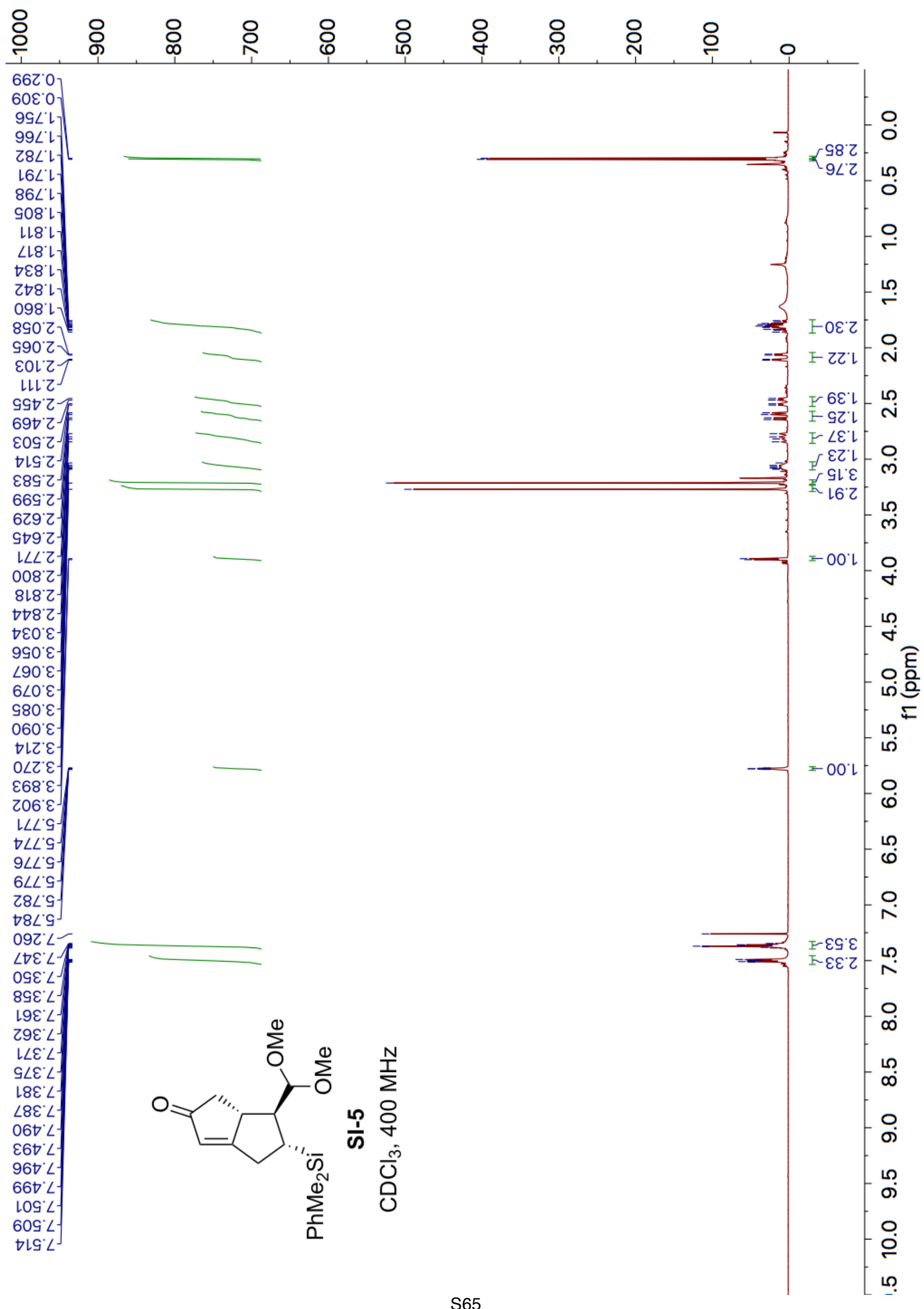




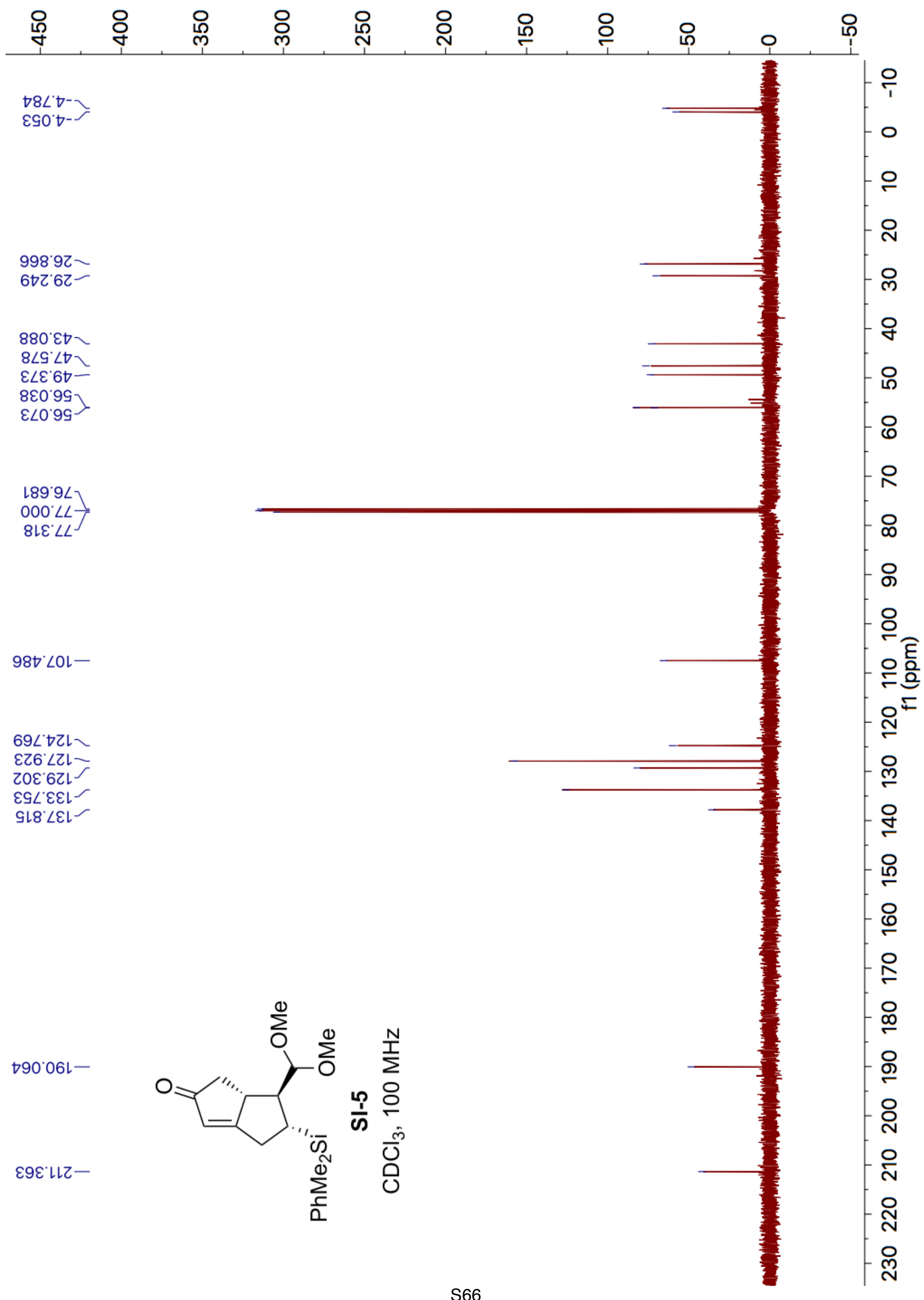




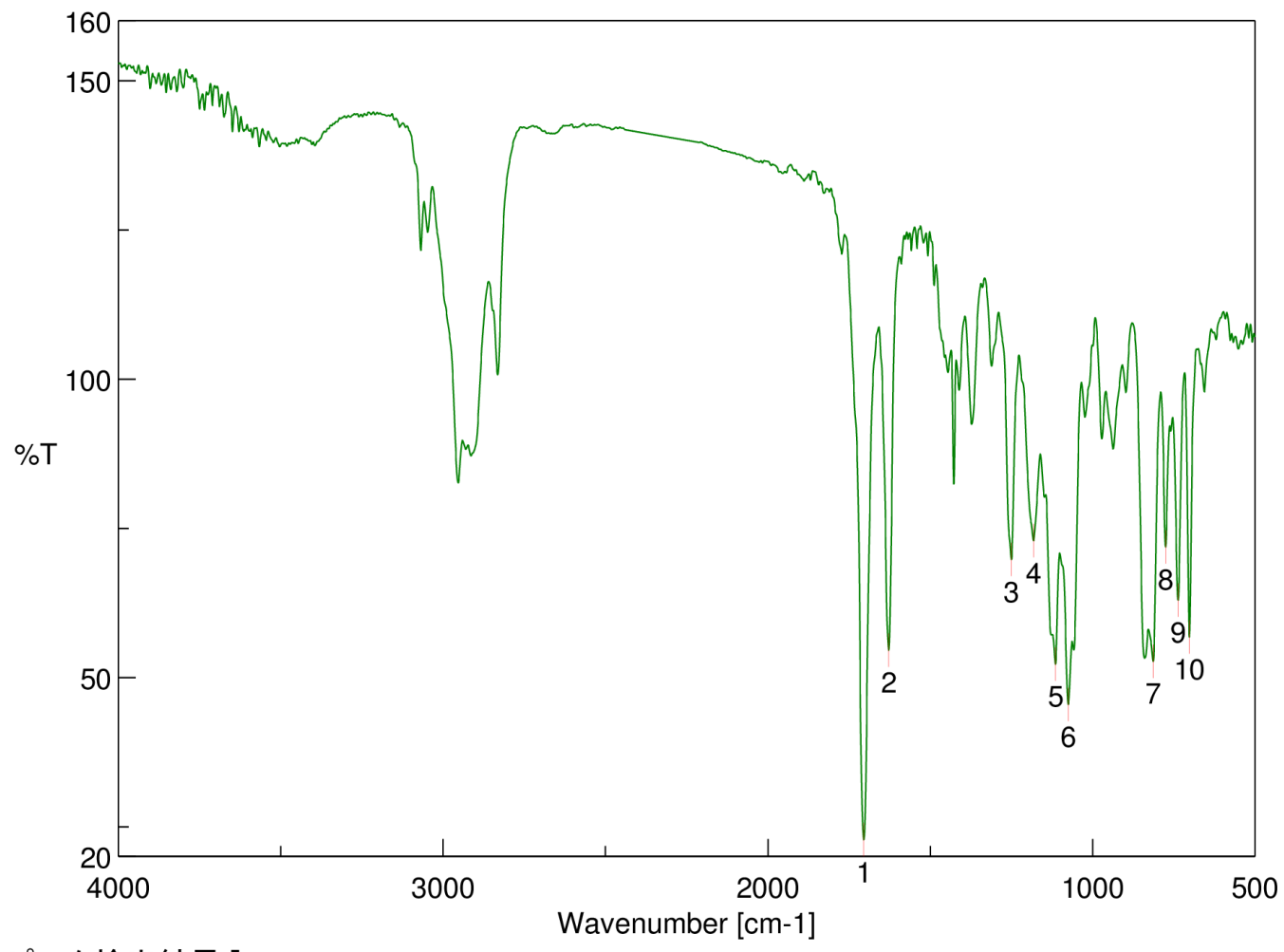

[ピーク検出結果 ]

$\begin{array}{llllll}\text { No. } & \text { 位置 } & \text { 強度 } & \text { No. } & \text { 位置 } & \text { 強度 } \\ 1 & 1704.76 & 22.7774 & 2 & 1627.63 & 54.5343 \\ 3 & 1250.61 & 69.7625 & 4 & 1182.15 & 72.8871 \\ 5 & 1114.65 & 52.238 & 6 & 1075.12 & 45.4676 \\ 7 & 813.813 & 52.6943 & 8 & 775.244 & 71.8284 \\ 9 & 736.674 & 62.9467 & 10 & 701.962 & 56.751\end{array}$<smiles>COC(OC)[C@@H]1[C@@H]2CC(=O)C=C2C[C@H]1[SiH3]</smiles> 


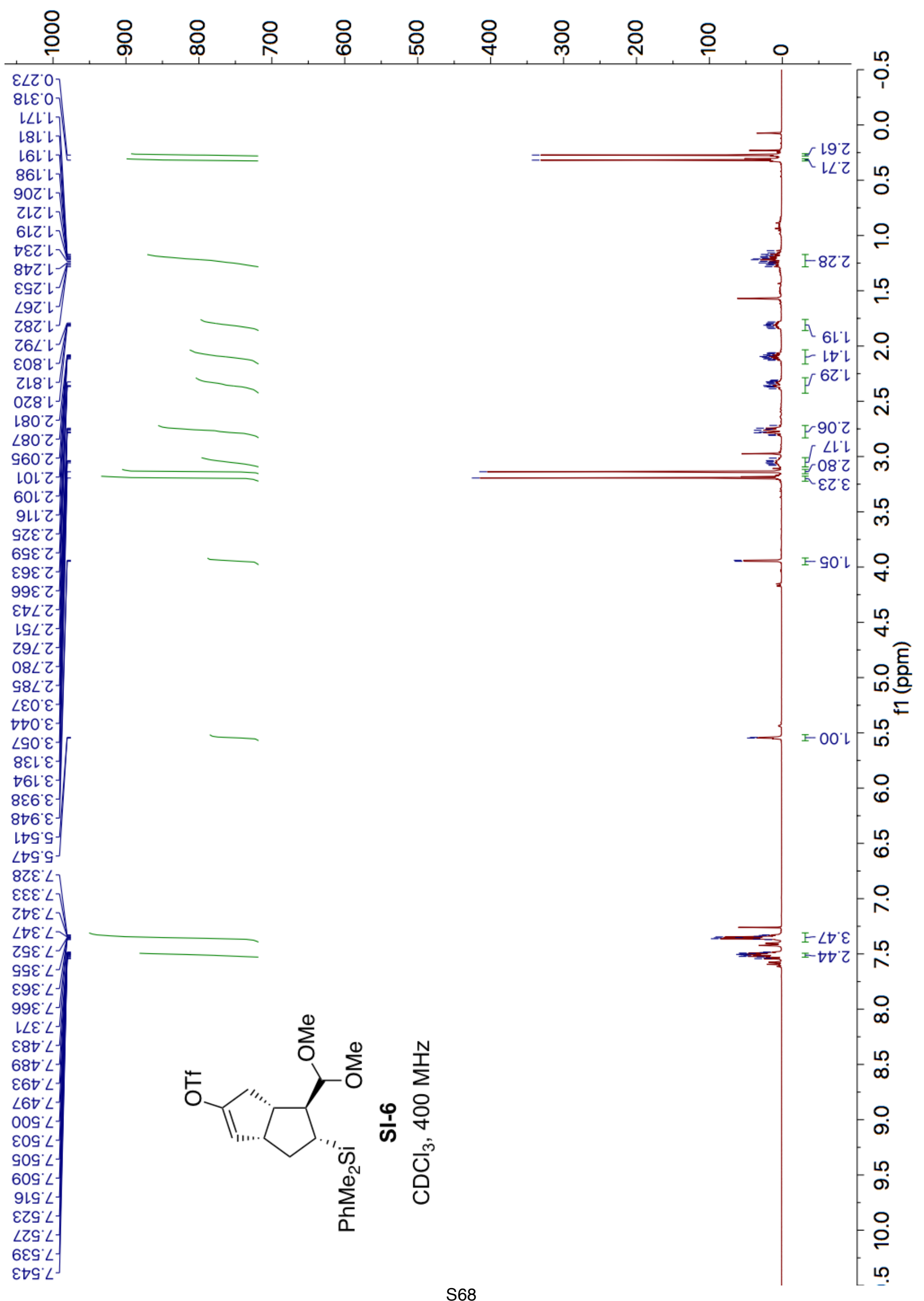




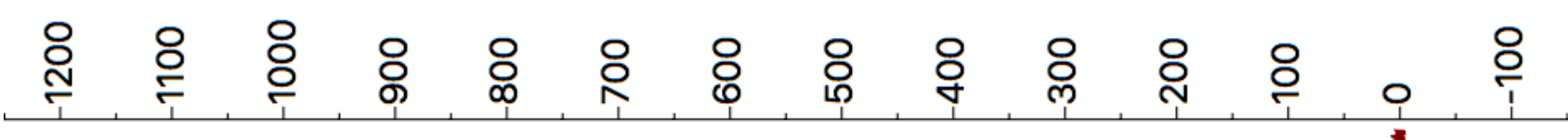

งャ6`元

เจE. $\varepsilon^{-}$

\&ฤฤ:8乙-

$066^{\circ} \mathrm{GE}$

$8 \angle 9^{\circ} \angle E-$

69l'LD -

Z90.97

แLE'EG]

68L'GS]

996.99-

$289^{\circ} 9 L$

$000^{\circ} \angle L \frac{1}{5}$

$6 L \varepsilon^{\prime} L L$

$\varepsilon \varepsilon 9^{\circ} \mathrm{LOL}-$

$\left.\begin{array}{l}\text { l9l:LZL } \\ 99 L \angle Z L\end{array}\right]$

$0 Z 6.8 Z 1]$

996.6ZL

$\angle 96^{\circ} 0 \varepsilon L-$

$060^{\circ}$ ZEL

$989^{\circ} \varepsilon \varepsilon L^{\circ}$

$\angle L 6{ }^{\circ} 8 L^{\circ}$

LL8'9tL-
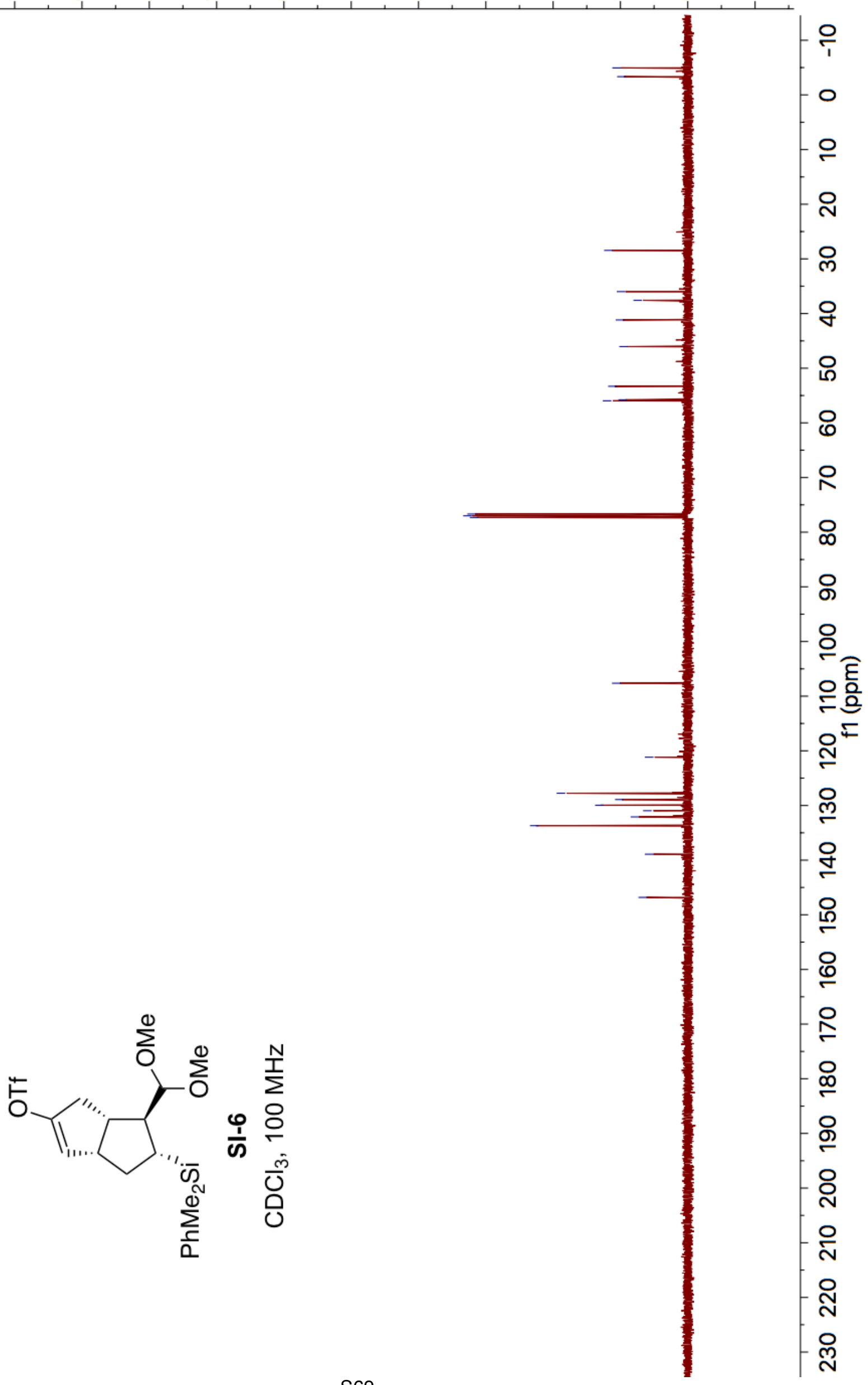


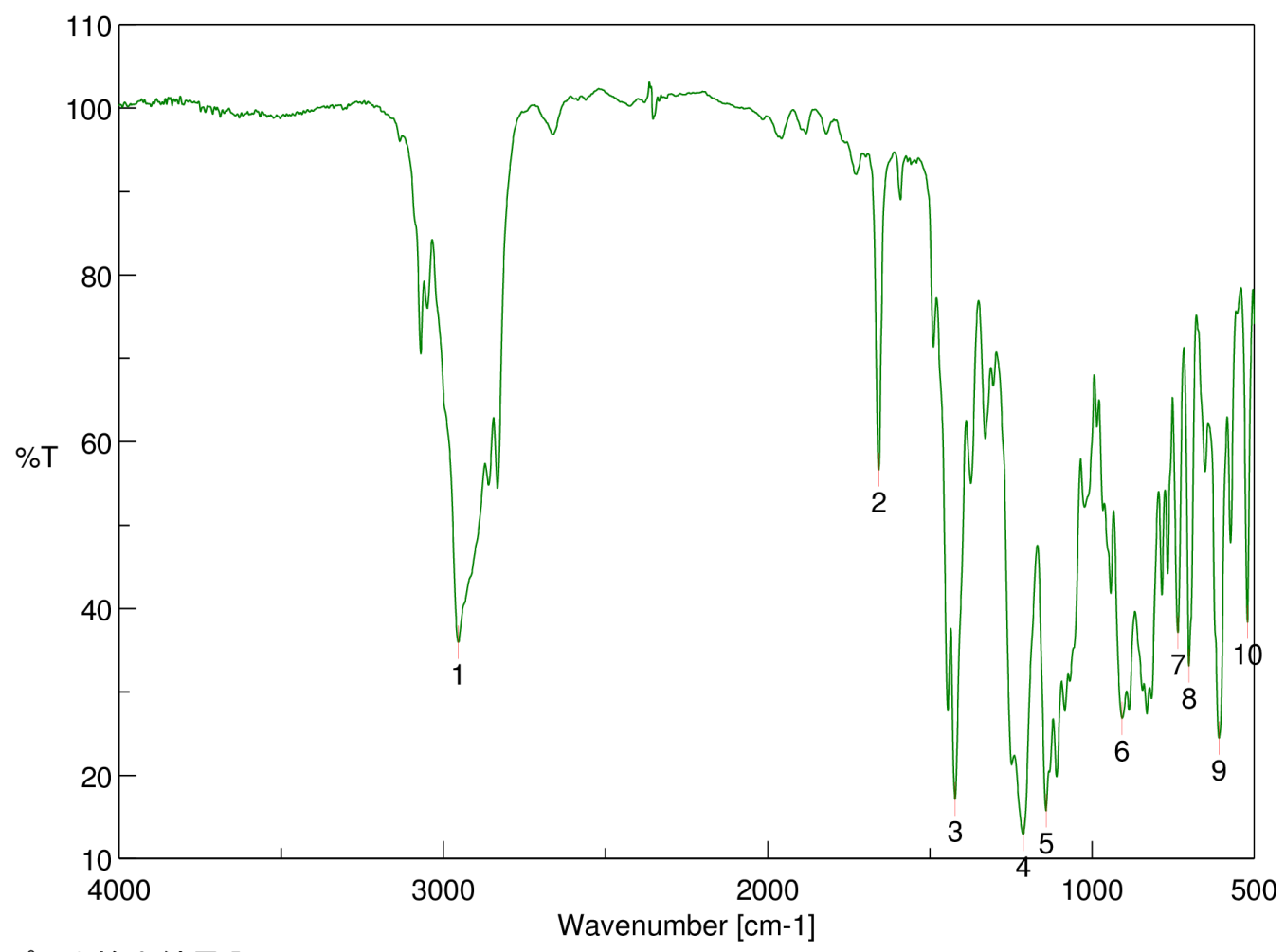

[ピーク検出結果 ]

$\begin{array}{llllll}\text { No. } & \text { 位置 } & \text { 強度 } & \text { No. } & \text { 位置 } & \text { 強度 } \\ 1 & 2954.41 & 35.9417 & 2 & 1657.52 & 56.5962 \\ 3 & 1422.24 & 17.0954 & 4 & 1212.04 & 12.9247 \\ 5 & 1141.65 & 15.7514 & 6 & 907.344 & 26.8217 \\ 7 & 735.71 & 37.1148 & 8 & 700.998 & 33.0782 \\ 9 & 608.431 & 24.4468 & 10 & 520.686 & 38.3283\end{array}$<smiles>COC(OC)[C@@H]1[C@H]([SiH3])C[C@@H]2C=C(O[Ga])C[C@H]21</smiles> 
品

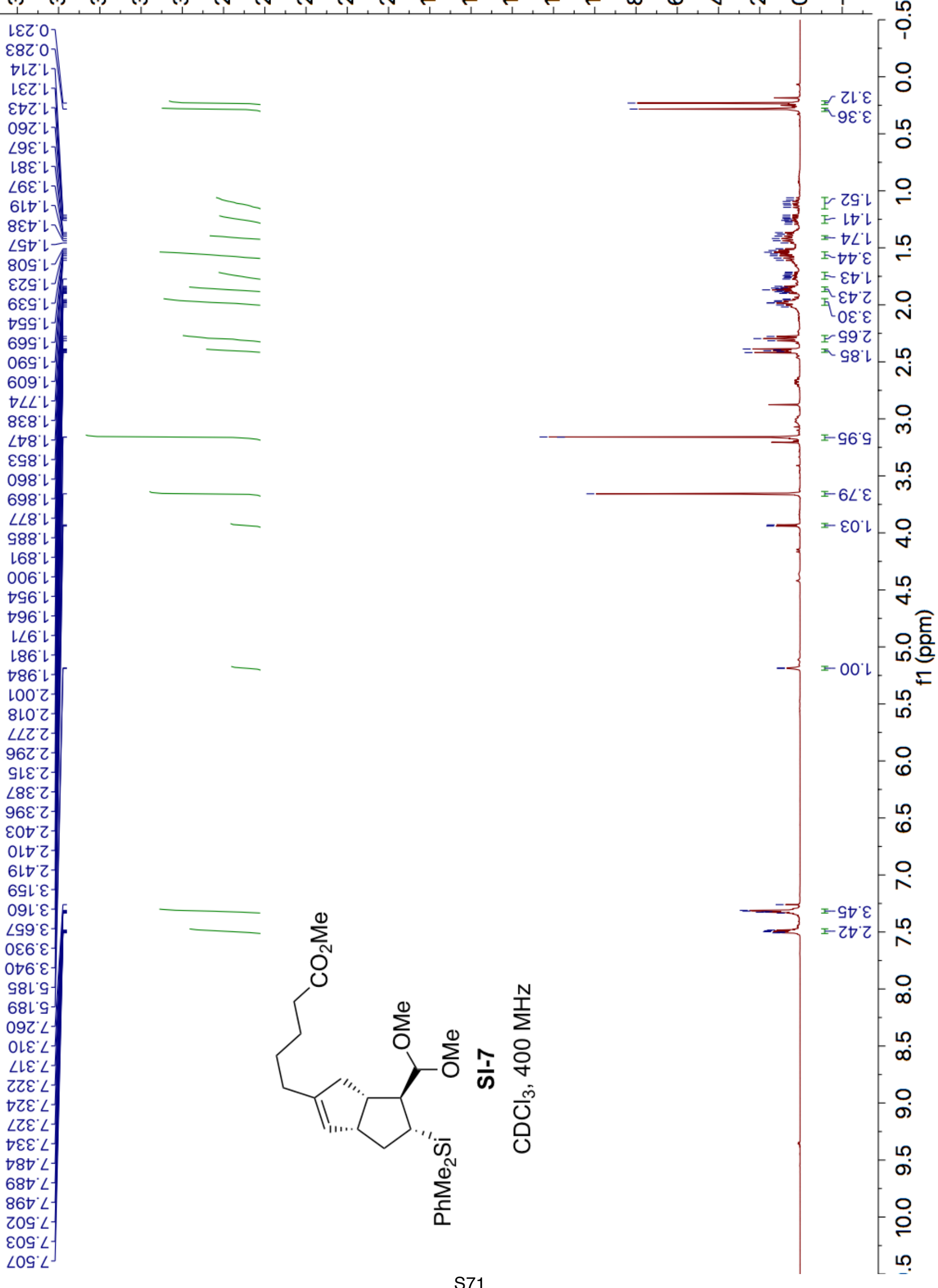




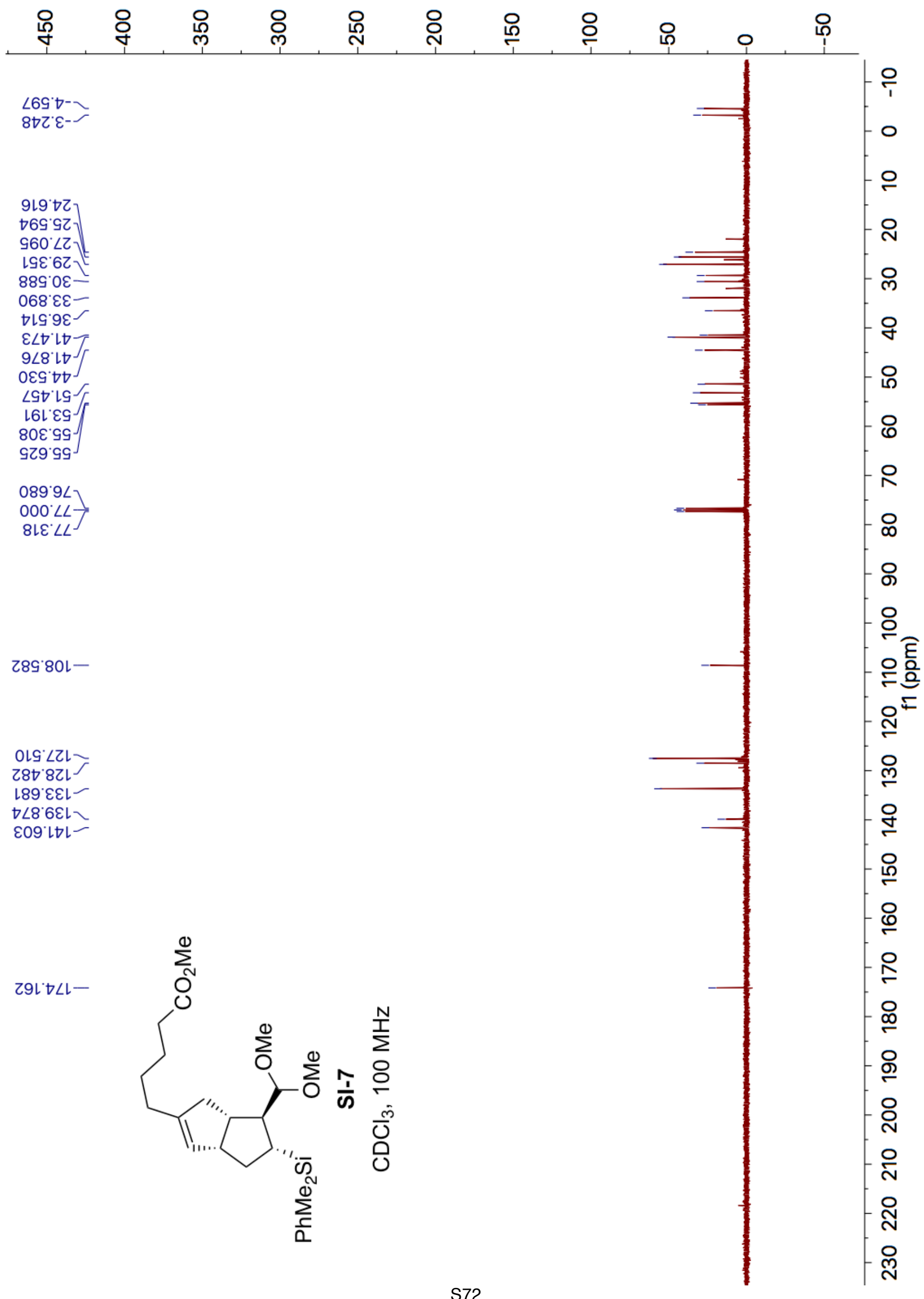




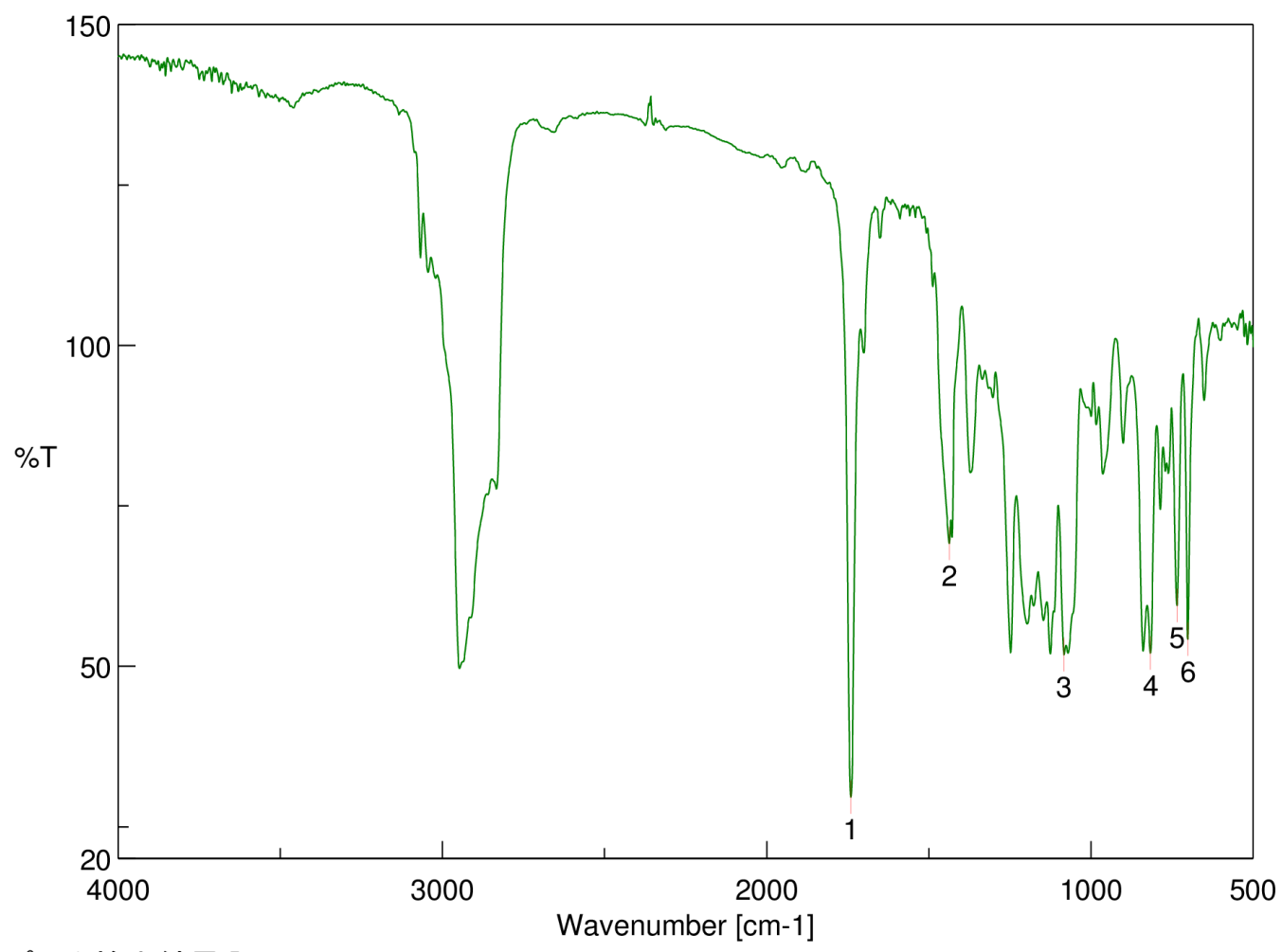

[ピーク検出結果 ]

$\begin{array}{llllll}\text { No. } & \text { 位置 } & \text { 強度 } & \text { No. } & \text { 位置 } & \text { 強度 } \\ 1 & 1740.44 & 29.5842 & 2 & 1436.71 & 69.108 \\ 3 & 1082.83 & 51.7619 & 4 & 816.706 & 52.0418 \\ 5 & 734.746 & 59.4168 & 6 & 700.998 & 54.164 \\ & & & & & \\ \mathrm{CO}_{2} \mathrm{Me} & & & & \end{array}$


兽 离

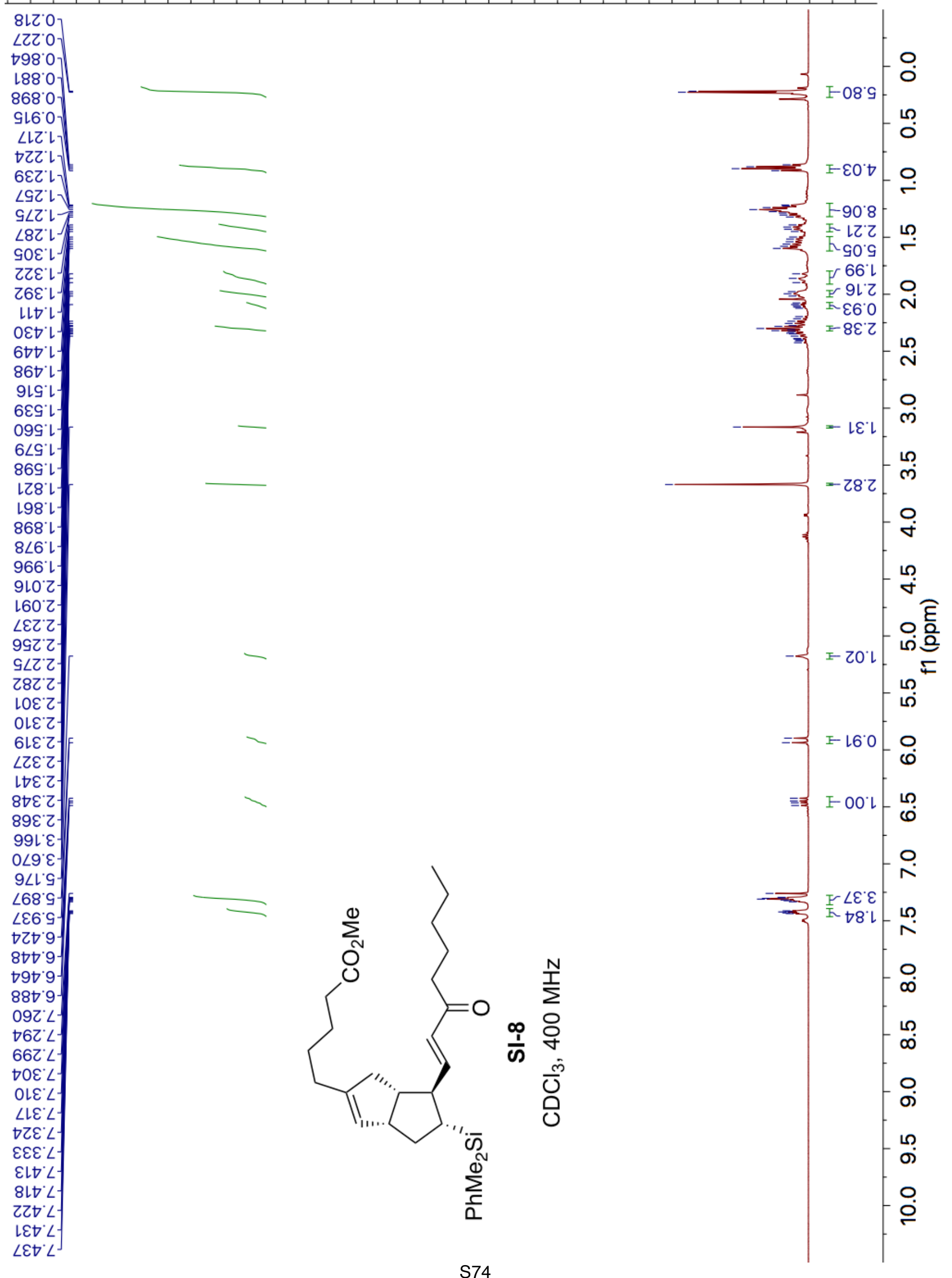




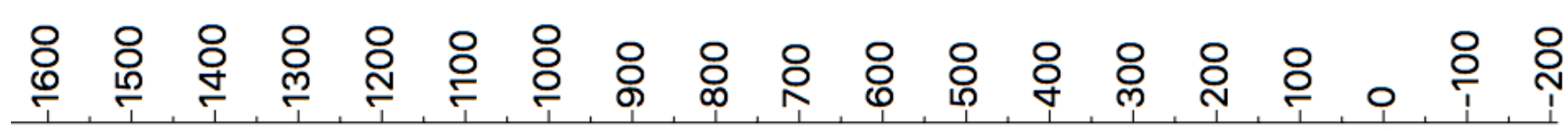

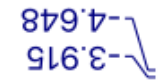

GG6. $\varepsilon$ -

เ8૪ 乙乙

ง $8 L^{\circ} \varepsilon 乙$

LSG $\nabla Z$

$\left.Z 90^{\circ} \mathrm{LZ}\right]$

G09. $0 \varepsilon$

เ8๋ $เ \varepsilon$

L $\mathcal{G}^{\prime} L \varepsilon_{-}$

乙98 $\varepsilon \varepsilon$

เ89'†E

ง99' $9 \varepsilon$

$\varepsilon เ \varepsilon^{\prime} 8 \varepsilon$

จL'6E

8จ0'L

997'ls

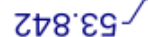

$189^{\circ} 9 \angle$

$000 \angle L T$

$81 \varepsilon^{\prime} L L$

GZ9 $\angle$ LL

LZL'8ZL

998.8ZL-

乙EL'6乙L

$0 \varepsilon 88^{\circ} \varepsilon \varepsilon \iota^{\circ}$

Z0† $8 \varepsilon L$ J

990เレL-

966.09L-

レてレナレレー

968.00Z-

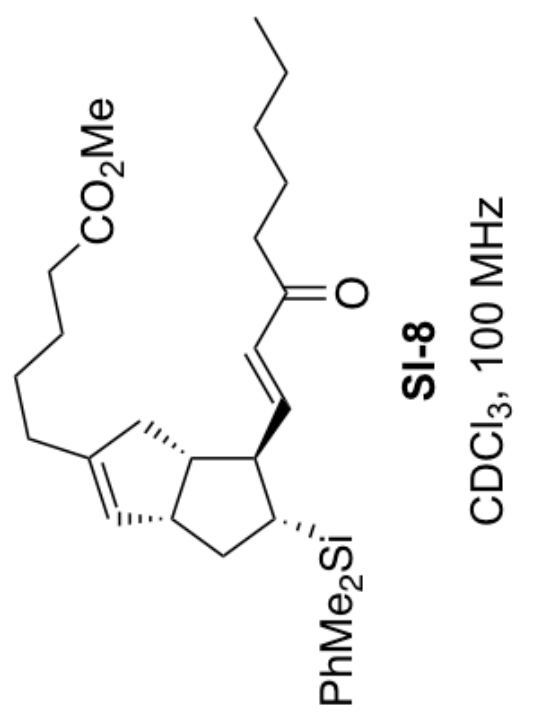

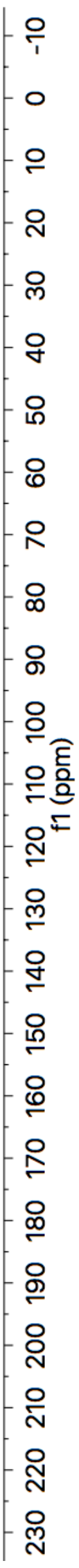




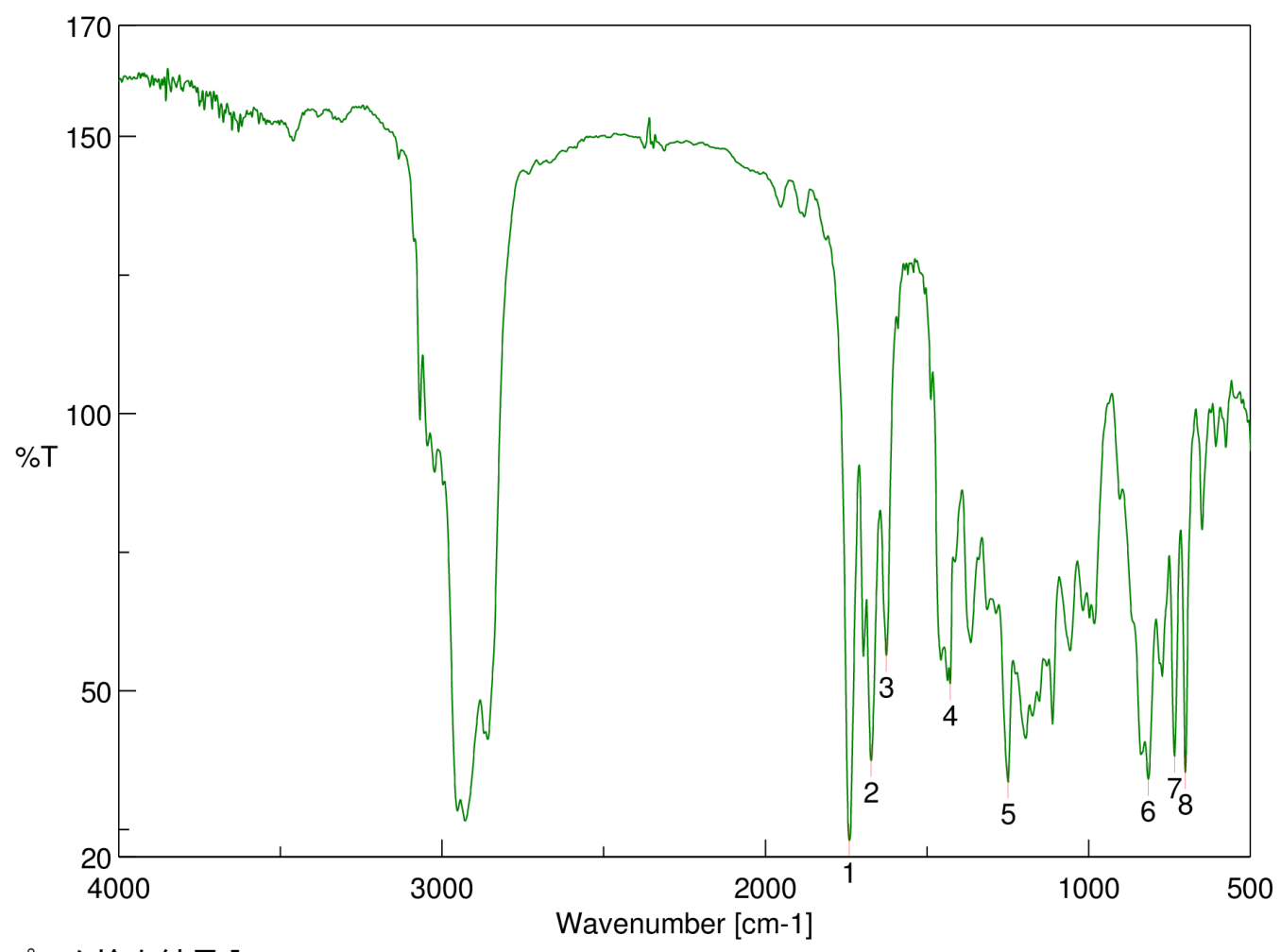

[ピーク検出結果 ]

$\begin{array}{llllll}\text { No. } & \text { 位置 } & \text { 強度 } & \text { No. } & \text { 位置 } & \text { 強度 } \\ 1 & 1740.44 & 22.9845 & 2 & 1672.95 & 37.3902 \\ 3 & 1625.7 & 56.4043 & 4 & 1428.03 & 51.2533 \\ 5 & 1249.65 & 33.5348 & 6 & 815.742 & 34.0608 \\ 7 & 734.746 & 38.2204 & 8 & 700.998 & 35.2878\end{array}$




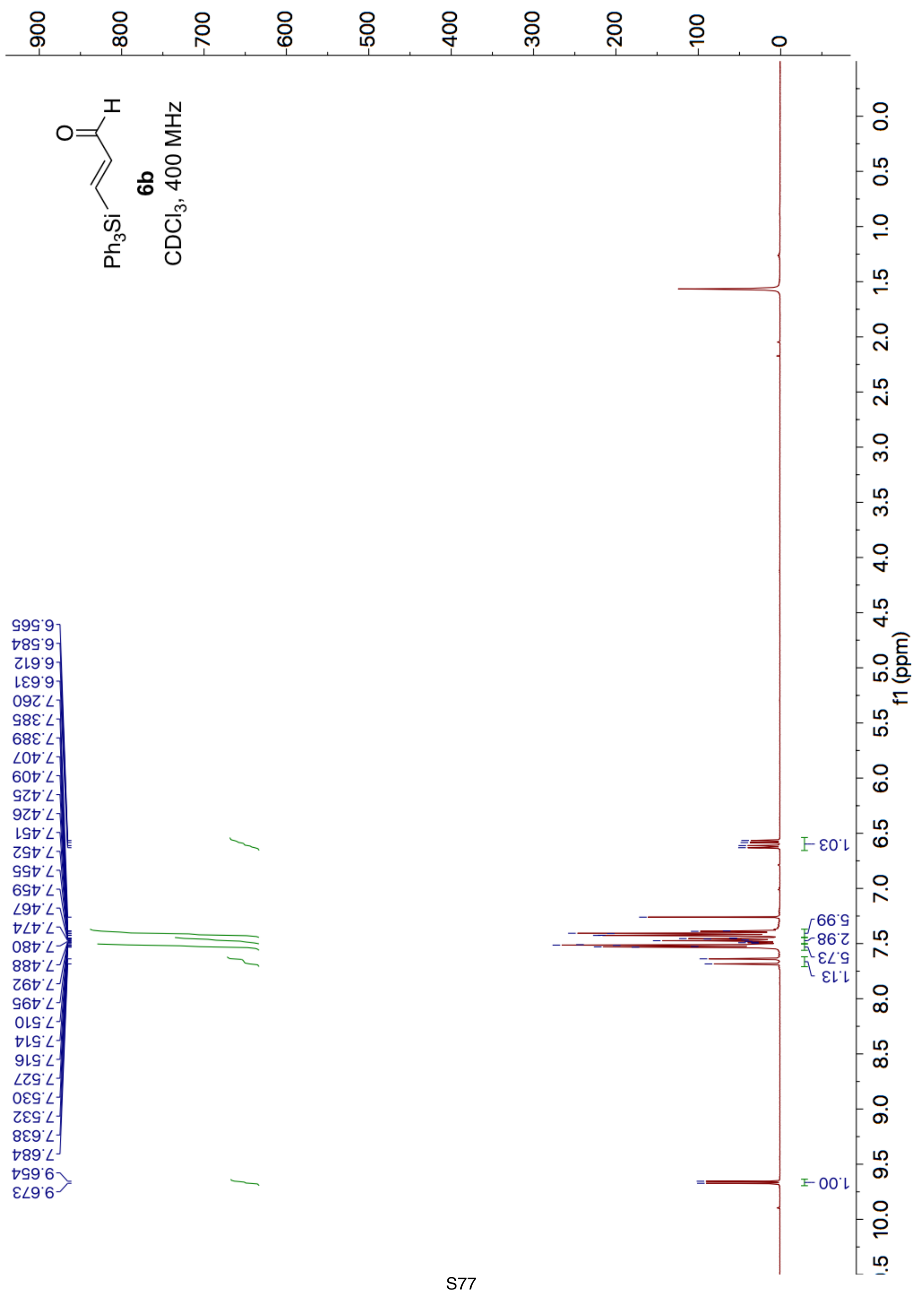




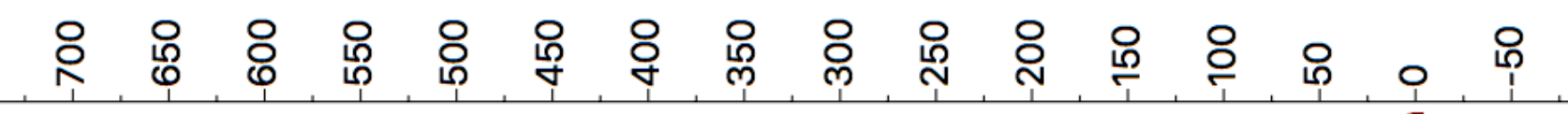

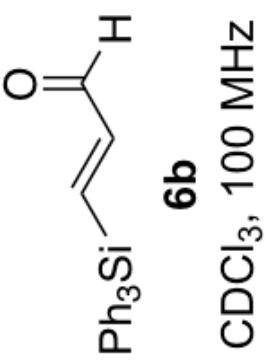

OtZ'8ZL ר

LLZ'OEL-

เ66'Lレ

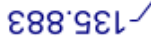

$9 \varepsilon \angle ゙ \angle \nabla ー$

6てャてGレー

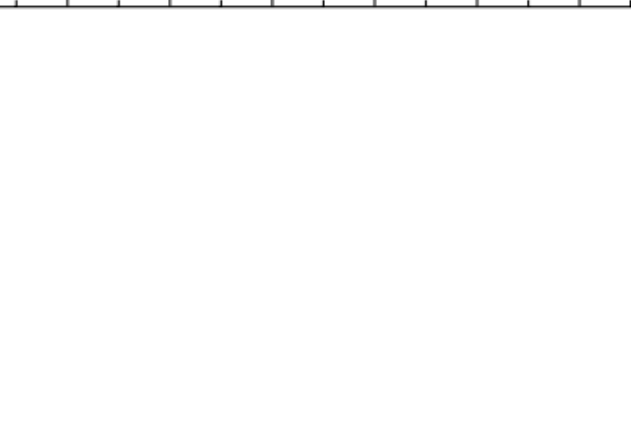




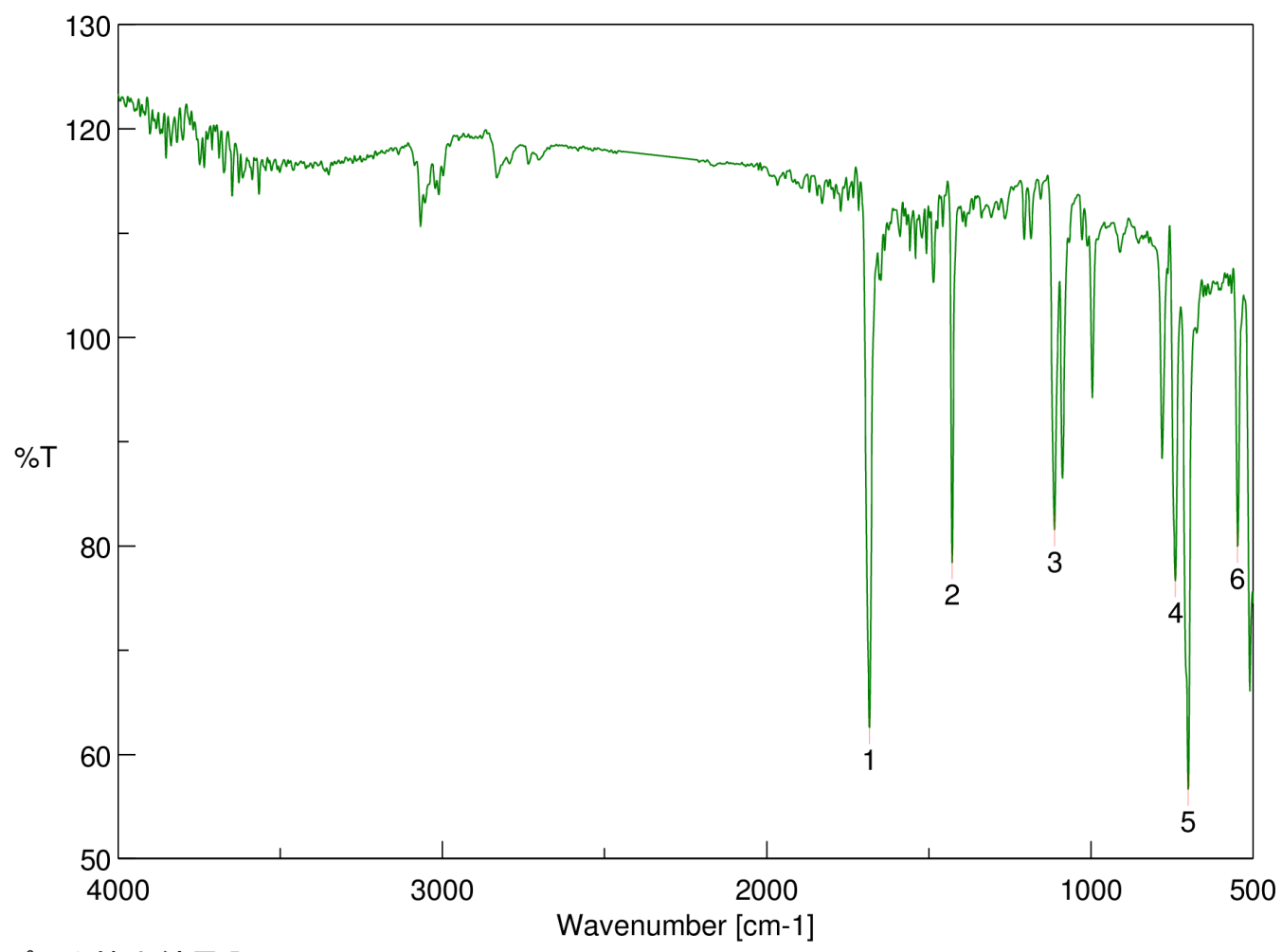

[ピーク検出結果 ]

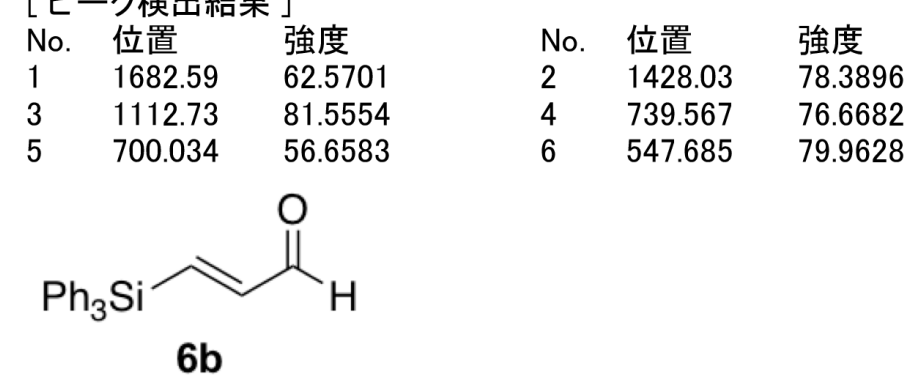




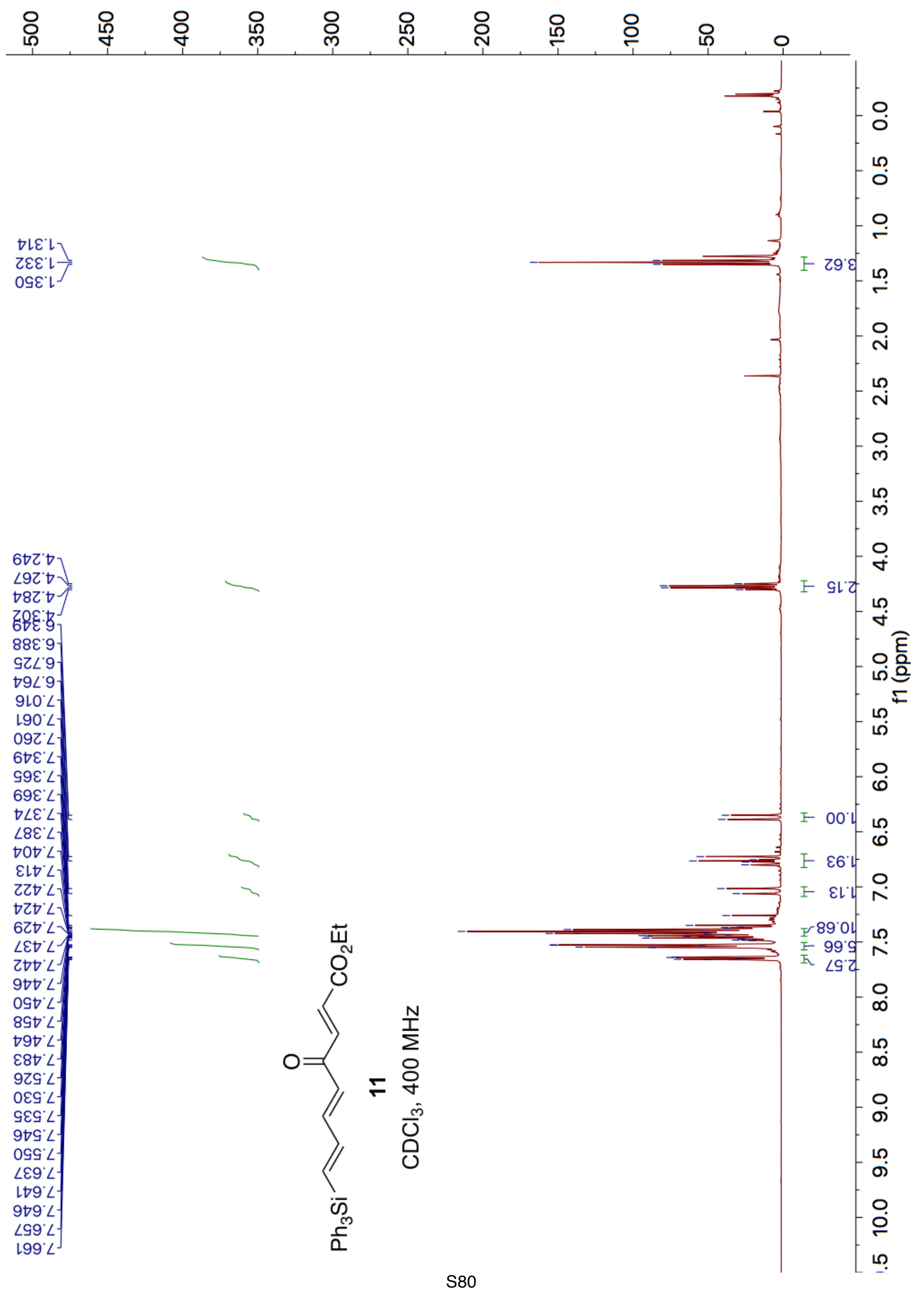




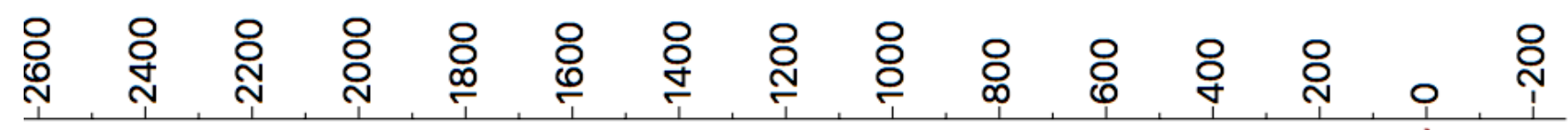

L60'tレー

8E\&'เ9-

189.94

$000: \angle L T$

$6 L E^{\prime} L L$

$798^{\circ} \angle Z L$

$990^{\circ} 8 \mathrm{ZL}$

$\varepsilon Z 66^{\circ} 6 \mathrm{Zl}$

$8 \varepsilon 0^{\circ} 0 \varepsilon l-$

$\varepsilon 9 l^{\circ} \varepsilon \varepsilon L^{-}$

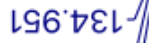

$\angle L 8 \cdot 9 \varepsilon l$

เSL'8EL

Z69'Lt-

GL6'9tL

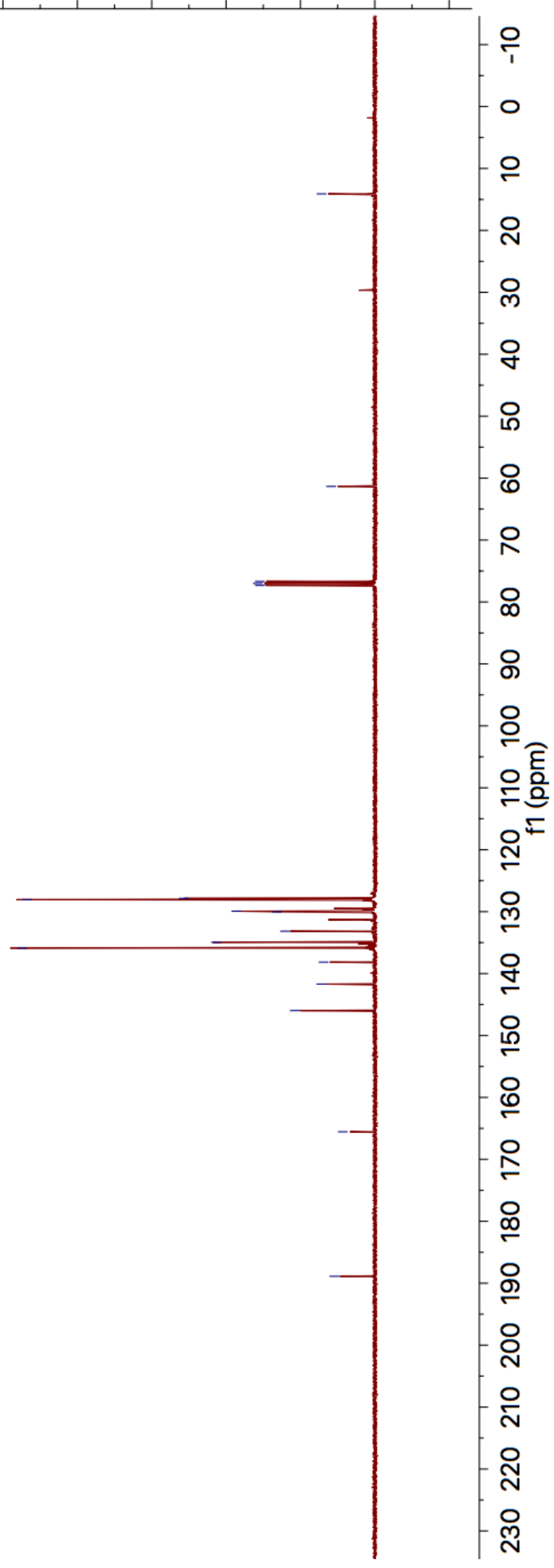




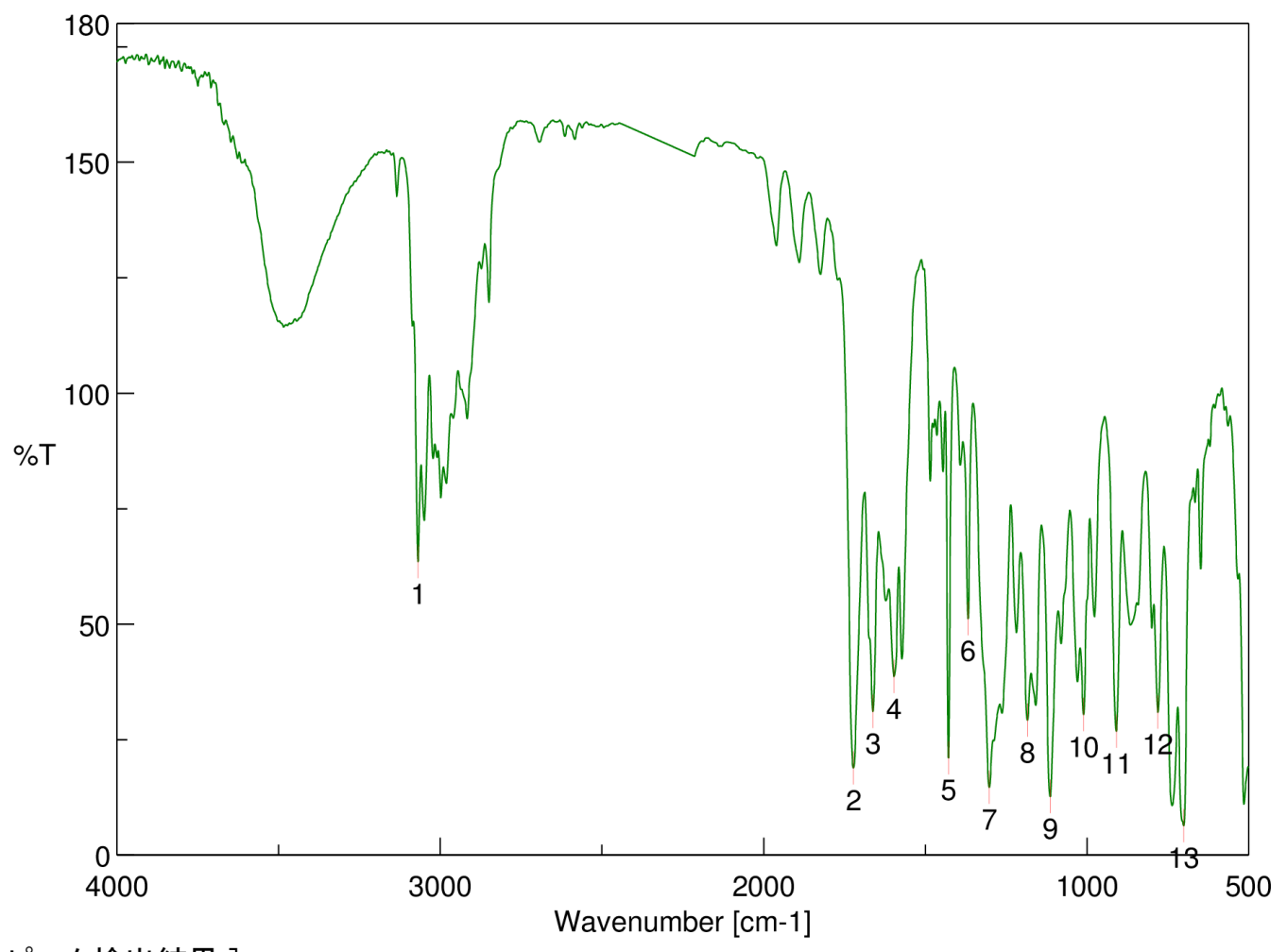

[ピーク検出結果 ]

$\begin{array}{llllll}\text { No. } & \text { 位置 } & \text { 強度 } & \text { No. } & \text { 位置 } & \text { 強度 } \\ 1 & 3069.16 & 63.5045 & 2 & 1722.12 & 18.9096 \\ 3 & 1662.34 & 31.0955 & 4 & 1596.77 & 38.6783 \\ 5 & 1428.03 & 21.0662 & 6 & 1368.25 & 51.1504 \\ 7 & 1302.68 & 14.71 & 8 & 1184.08 & 29.2176 \\ 9 & 1113.69 & 12.6375 & 10 & 1010.52 & 30.3864 \\ 11 & 909.272 & 26.8261 & 12 & 781.029 & 30.9357 \\ 13 & 700.998 & 6.38946 & & & \end{array}$<smiles>CCOC(=O)/C=C/C(=O)/C=C/C=C/[SnH+]c1ccccc1</smiles> 